University of Louisville

ThinkIR: The University of Louisville's Institutional Repository

Electronic Theses and Dissertations

$8-2020$

\title{
Methods for Mass Data Acquisition of Whiskey Webs.
}

Adam D. Carrithers

University of Louisville

Follow this and additional works at: https://ir.library.louisville.edu/etd

Part of the Applied Mechanics Commons, and the Computer-Aided Engineering and Design Commons

\section{Recommended Citation}

Carrithers, Adam D., "Methods for Mass Data Acquisition of Whiskey Webs." (2020). Electronic Theses and Dissertations. Paper 3360.

https://doi.org/10.18297/etd/3360

This Master's Thesis is brought to you for free and open access by ThinkIR: The University of Louisville's Institutional Repository. It has been accepted for inclusion in Electronic Theses and Dissertations by an authorized administrator of ThinkIR: The University of Louisville's Institutional Repository. This title appears here courtesy of the author, who has retained all other copyrights. For more information, please contact thinkir@louisville.edu. 


\title{
METHODS FOR MASS DATA ACQUISITION OF WHISKEY WEBS
}

\author{
By \\ Adam D. Carrithers \\ B.S. Mechanical Engineering, University of Louisville, 2019
}

\author{
A Thesis \\ Submitted to the Faculty of the \\ University of Louisville \\ J. B. Speed School of Engineering \\ as Partial Fulfillment of the Requirements \\ for the Professional Degree \\ MASTER OF ENGINEERING \\ Department of Mechanical Engineering
}

July 2020 
Copyright 2020 by Adam David Carrithers

All rights reserved 



\section{METHODS FOR MASS DATA ACQUISITION OF WHISKEY WEBS}

Submitted by:

Adam D. Carrithers

A Thesis Approved on

July 24,2020

by the following Reading and Examination Committee:

Stuart J. Williams, Ph.D., Thesis Director

Ellen G. Brehob, Ph.D.

James C. Watters, Ph.D. 


\section{ACKNOWLEDGEMENTS}

I am deeply appreciative of my parents, David and Stacie Carrithers, for their unwavering support in all my endeavors. I could not have made it this far without their guidance and encouragement. I will always be indebted.

I would like to thank my thesis director, Stuart Williams, for the opportunity to work on this research both as an undergraduate and graduate researcher. I regard him highly as both an educator and mentor and am grateful for his help in completing this work. I am appreciative of Mohamed "Mido" Rashed, Martin "Marty" Brown VI, and Md Mahmudur "Rony" Rahman for their friendship, ideas, and advancement of my knowledge and skills in the lab. I would also like to thank the reading committee, Dr. Brehob and Dr. Watters, both of whom I admire.

I would like to give special thanks to the kind folks at Funai Lexington Technology Corporation for providing instrumentation, as well as technical support and access to their facilities to complete my research.

Thanks to Nicole Hartman for her encouragement and help with coding.

To my brother, Drew, and sister, Amanda, I appreciate your ability to provide comic relief when I become overly distressed. Although we are growing up and paving our own ways, you will always be close in my heart. 


\author{
ABSTRACT \\ Methods for Mass Data Acquisition of Whiskey Webs \\ Adam D. Carrithers
}

July 24, 2020

Whiskey webs are a recently discovered phenomenon in which the monolayer collapse during evaporation of diluted American whiskey results in self-assembled, weblike structures. It was found that the pattern of weblike structures were unique between different American whiskey products. It is desirable to use these unique patterns as a "fingerprint" which can be used to identify whiskeys, their chemical constituents, and perhaps even counterfeits. In pursuit of identifying whiskey products based on their web pattern, it is necessary to have a large database of samples imaged for each product. The device discussed herein is the realization of an apparatus capable of automating the droplet deposition and imaging of whiskey webs. The apparatus was shown to produce droplets with a smaller volumetric deviation than traditional methods. The apparatus will standardize the droplet deposition procedure and serve as the foundation for statistical studies of whiskey web uniqueness and chemical characterization. 
TABLE OF CONTENTS

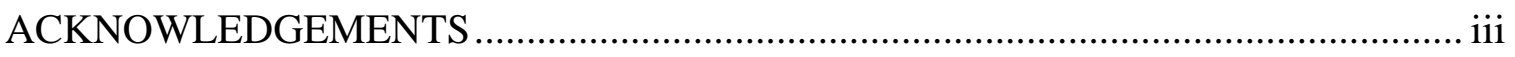

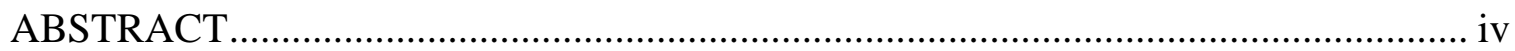

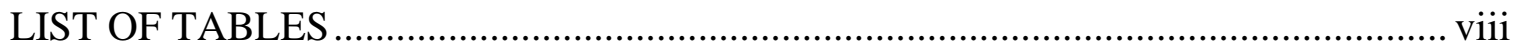

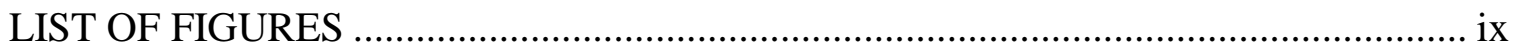

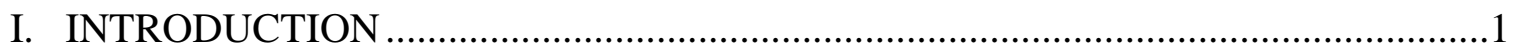

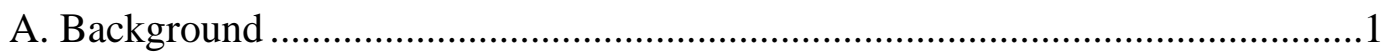

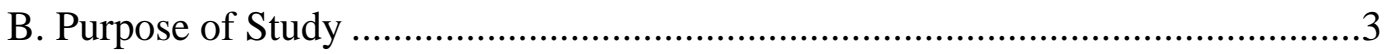

C. Significance of Study ………………………...............................................

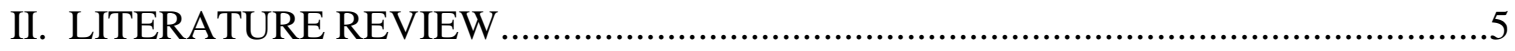

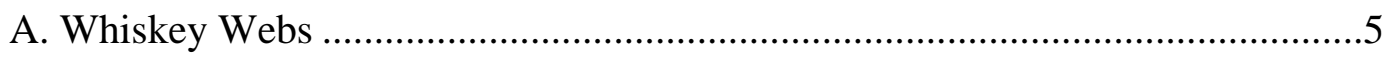

B. Image Pattern Recognition ...........................................................................10

D. Thermal Inkjet Printing ..........................................................................12

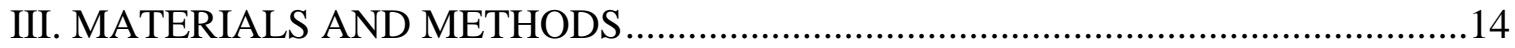

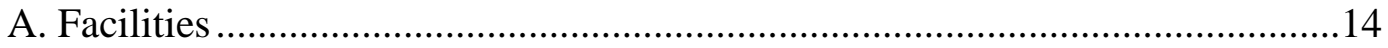

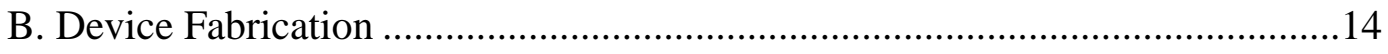

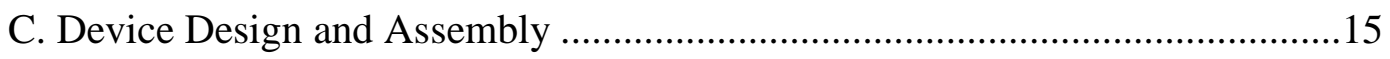

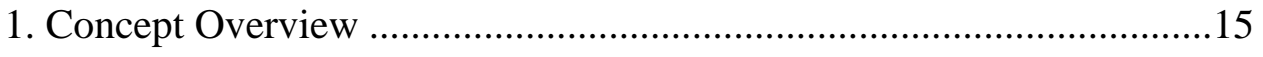

2. Printing Subsystem ……………………………………………….....19

3. Tape Transport Subsystem...................................................................22

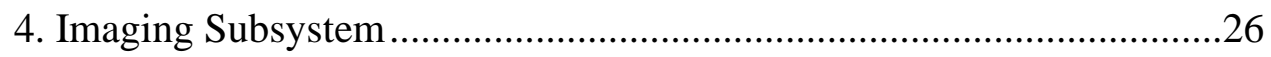

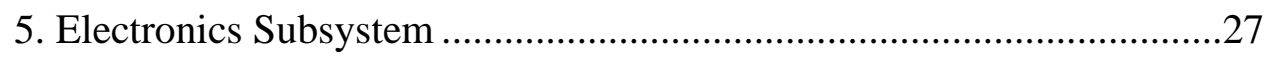

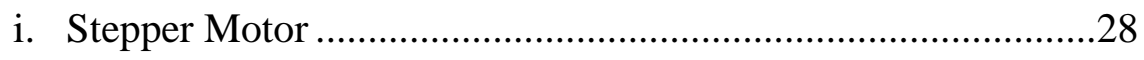




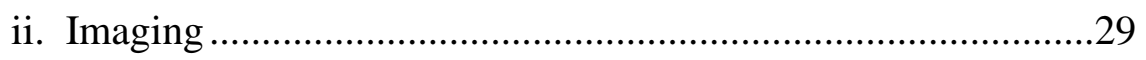

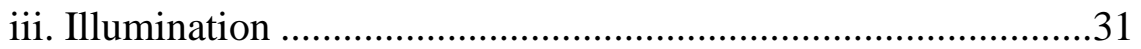

iv. Temperature and Humidity Sensing ...........................................32

6. Coding and Device Timing ..................................................................32

D. Whiskey Sample Analysis ..............................................................................43

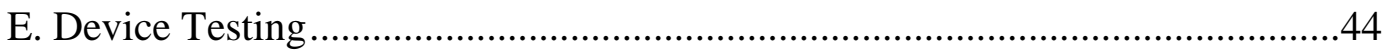

1. Cartridge Selection..................................................................................

2. Image Focus and Digital Analysis .......................................................46

3. Droplet Illumination...........................................................................50

4. Temperature and Humidity Sensor Validation ........................................52

5. Stepper Motor Accuracy Validation .......................................................52

6. Film Tension Test …………………………………………………....53

7. Printhead Failure Analysis ..................................................................56

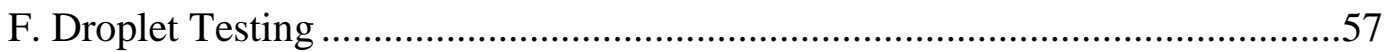

1. Manual Droplet Testing .........................................................................58

2. Continuous Droplet Testing .................................................................58

3. Single Droplet Testing ...........................................................................59

4. Single Droplet Testing: Single Nozzle Variant …………………….....61

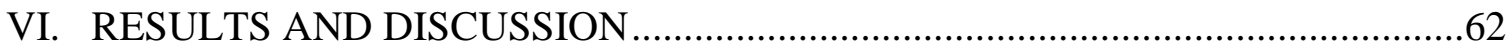

A. Temperature and Humidity Sensor Validation ................................................62

B. Continuous Drop Test I ................................................................................63

C. Stepper Motor Accuracy Validation ................................................................64

D. Film Tension Test ……………………………....................................65

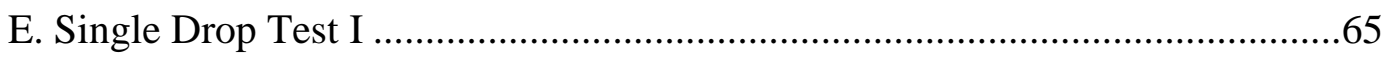

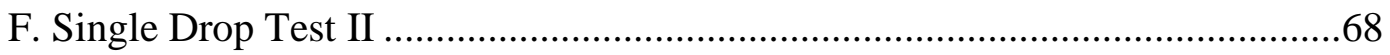

G. Cartridge Failure Analysis ……………………..........................................

H. Single Droplet Testing: Single Nozzle Variant..................................................73 


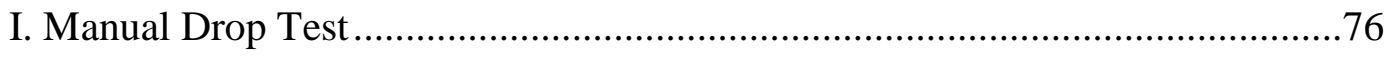

J. Continuous Drop Test II ................................................................................

K. Deposition Method Comparison .......................................................................79

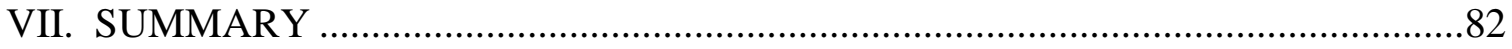

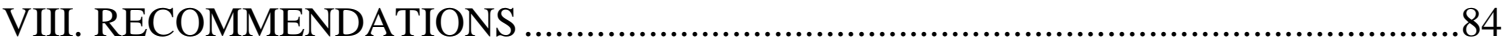

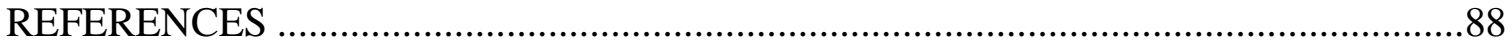

\section{APPENDIX}

A. Full Text of Multiscale Self-Assembly of Distinctive Weblike Structures from Evaporated Drops of Dilute American Whiskeys .....................................................93

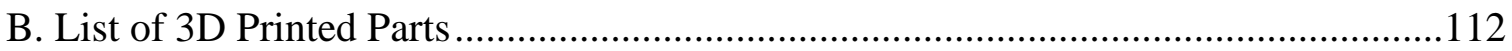

C. Detailed Apparatus Assembly ................................................................................113

D. Stepper Motor Specification ............................................................................124

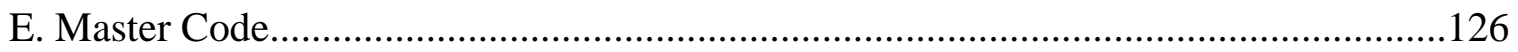

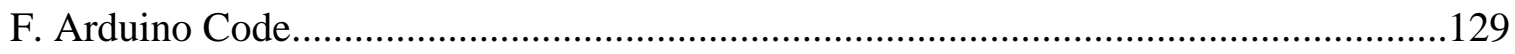

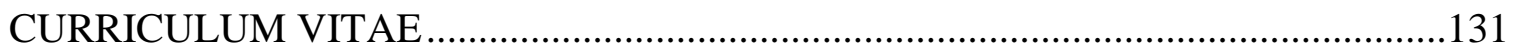




\section{LIST OF TABLES}

Table 1. Printing parameters for FCL Controller...................................................... 21

Table 2. Timing of functions for startup, steady state, and shutdown sequences for a travel distance of 115.5 millimeters, 7 millimeters between droplet centers, and assumed

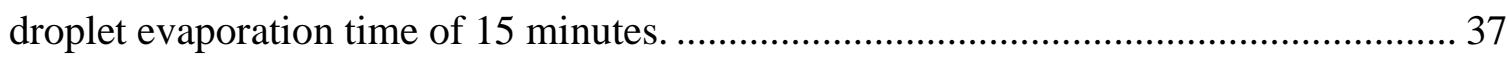

Table 3. Surface tension, viscosity, and pH for Buffalo Trace Kentucky Straight Bourbon Whiskey.

Table 4. Estimated droplet diameter vs. measured droplet diameter using objective mounted to telescopic lens. Focal length of lens was finely adjusted until droplet size was calibrated. .

Table 5. Relative humidity and temperature readings made by the ThermoPro and

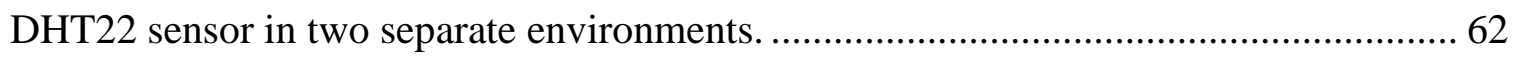

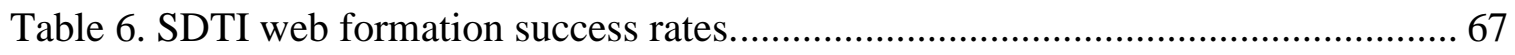

Table 7. Controller parameters for SDTII, SNV, and CDTII.................................... 80

Table 8. Comparison of droplet size and web formation success rate between the manual deposition technique, continuous dropping with an entire primitive, and single drop testing with a single nozzle. 


\section{LIST OF FIGURES}

Figure 1. Whiskey webs formed with American whiskeys diluted to 20-25\% alcohol by volume: a) Old Rip Van Winkle 10 Year, b) Four Roses Single Barrel, c) Baker's Bourbon, d) Van Winkle Special Reserve 12 Year, e) O.K.I. 8 Year, f) Woodford Reserve Double Oaked. Scale bar is 0.5 millimeters........................................................ 6

Figure 2. Effect of congeners on whiskey web patterns. Various congeners were added to a control bourbon whiskey to demonstrate that the concentration and combination of these compounds ultimately guide each whiskey pattern................................................. 8

Figure 3. Analysis of microscope images of web patterns. Sample A) Old Weller Antique, B) W. L. Weller Special Reserve, C) W. L. Weller 12 Year. ............................... 9

Figure 4. Retinal fundus image. Modified from (Williams, McGowan et al. 2015)....... 10

Figure 5. FCL Controller provided by Funai Lexington Technology Corporation........... 15

Figure 6. Rendered slide rail concept for semi-automation of whiskey web fabrication. 16 Figure 7. Front view of slide rail concept, depicting narrow tolerances between walls of aluminum tray and FCL Controller................................................................................. 17

Figure 8. Rendered reel-to-reel concept for full automation of whiskey ………............... 18

Figure 9. Short and tall printer cartridges provided by FLTC. ……................................ 20

Figure 10. Mockup of nozzle placement on printer cartridge provided by FLTC............ 22

Figure 11. Front view of tape transport subsystem.................................................... 23

Figure 12. Close-up of prototype displaying two brakes inducing drag on the supply reel.

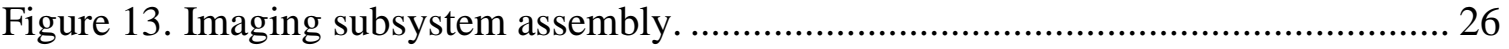

Figure 14. Motor mount designed to provide conductive heat …….................................. 29 
Figure 15. (Left) Do-it-yourself intervalometer prepared for connection to Arduino. (Right) Pinout for the 2.5-millimeter TRS plug.

Figure 16. DIY light ring made by adhering LED strips to a 3D printed holder and soldering terminals together.

Figure 17. Example Arduino code to demonstrate a serial connection with Python. Code waited for commands from Python and then executed the called function. 33

Figure 18. Example Python code to demonstrate serial connection with the Arduino.

Code sends commands to Arduino which are then executed. 34

Figure 19. Python example code for using subprocess module to control FCL controller.

Figure 20. Three states of the dispensing process: A) Droplets have been dispensed but have not yet reached the camera for imaging. B) Droplets have reached the camera for imaging and are still dispensing. C) All droplets have been dispensed but not all imaged.

Figure 21. Motor's total positional error for an 1 of 115.5 millimeters, 40

Figure 22. Time to dispense and collect 100 droplets during continuous operation. Assuming a droplet evaporation time of 15 minutes. ............................................... 42

Figure 23. Nikon objective mounted directly to Canon EOS 80D camera body............. 46

Figure 24. Uncropped images of droplets using a 4x objective lens (left) and 10x objective lens (right) mounted directly to camera body.

Figure 25. Uncropped image of droplet using 4x objective lens mounted to a telescopic lens to account for mechanical tube length of objective.

Figure 26. Images produced by adjusting camera shutter speed and position of LED ring light above the film at A) 1-inch, B) 2-inches, C) 3-inches, and D) 4-inches.

Figure 27. Stepper motor positional accuracy test setup. A) Start of test, tape aligned with datum. B) Several runs into test, tape is pulled forward by motor and the distance from the datum measured with digital calipers. 
Figure 28. A) Free body diagram of tension being measured by suspending a known weight from center of film. B) Weight being suspended from film in actual testing....... 54 Figure 29. Caps 3D printed to reduce evaporation from cartridge reservoir. .................. 57

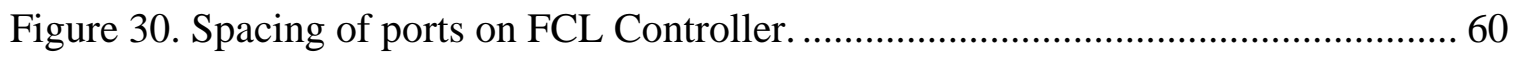

Figure 31. Positional error of stepper motor for 10 run commands............................... 64

Figure 32. SDTI. Filled markers indicate a whiskey web pattern formed. Unfilled markers

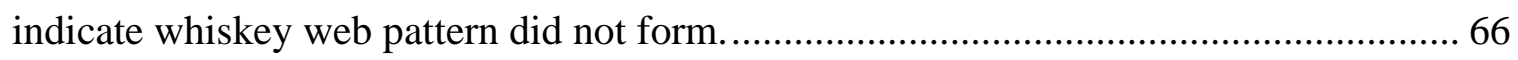

Figure 33. Effect of humidity on web formation. 2-millimeter wells. Woodford Reserve Double Oaked. A) 25\% RH, B) $45 \%$ RH, C) $71 \%$ RH.

Figure 34. SDTII. Filled markers indicate a whiskey web pattern formed. Unfilled markers indicate whiskey web pattern did not form. 69

Figure 35. A) 2.5x magnification and B) 20x magnification of left 71

Figure 36. A) 2.5x magnification and B) 20x magnification of left cartridge........ 72

Figure 37. Evaporation time and diameter of droplets for each nozzle address in SNV. Droplets were sorted by ascending evaporation time. Blue bars indicate a web pattern successfully formed while gray bars indicate a web pattern did not form. 73

Figure 38. SNV evaporated droplets imaged for A) Nozzle 6, and B) Nozzle 13.......... 74

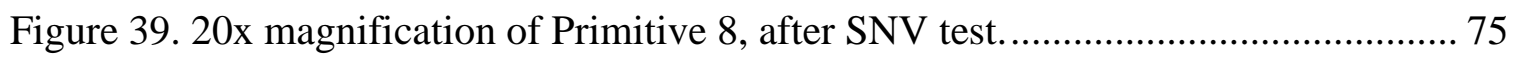

Figure 40. Evaporation time and diameter of manually deposited droplets. Droplets were sorted by ascending evaporation time. Blue bars indicate successful web formation..... 76 Figure 41. Droplet volumes of CTDII, sorted by ascending volume. Blue bars indicate a web pattern successfully formed while gray bars indicate a web pattern did not form.... 77 Figure 42. A) Droplet number 1 from CTDII test with a rounded square profile. B) Droplet number 10 from CTDII, representative of other droplets in the test. 78

Figure 43. Droplet images representative of the tests:.......................................... 79

Figure 44. (Left) Screw driven, manually adjustable linear stage ............................... 86 
Figure 45. Assembly of major mount to optical bread board.

Figure 46. Spacing major and minor mounts on an optical

Figure 47. A) Assembly of the imaging subsystem. B) Camera being mounted to imaging subsystem. Parts used are: 1) Lead screws, 2) adjustment nut, 3) 3D printed camera

mount adapter, 4) standard tripod mount attached to Canon EOS 80D. 115

Figure 48. Exploded view of the stepper motor assembly. 116

Figure 49. A) Exploded view of uptake reel subassembly. B) Timing belt being aligned with uptake reel gear and motor shaft adapter. C) Uptake reel subassembly being positioned to induce tension in the timing belt.

Figure 50. Leveling of supply reel holder with capstan.......................................... 118

Figure 51. FEP film mounted to the supply reel holder so that the 119

Figure 52. A) Assembly of the braking subsystem for the reel. B) Braking system being mounted to apparatus. Parts used are: 1) 3D printed supply reel cap, 2) 3D printed supply reel brake, 3) self-adhesive clear rubber bumpers, and 4) minor mount for supply reel. 120 Figure 53. Illustration of tape being threaded through system. A) Film passing over nylon sleeve bearing. B) Film passing over capstan and secured to uptake reel. 120

Figure 54. Uptake reel cap securing uptake reel to shaft.

Figure 55. 1) 3D printed pinch roller pivot, 2) 3D printed pinch roller holder, 3) 3D

printed spring compressor, 4) compression spring, 5) neoprene pinch roller. 122

Figure 56. Final assembly of reel-to-reel apparatus 


\section{CHAPTER I. INTRODUCTION}

\section{$\underline{\text { A. Background }}$}

Although a seemingly mundane phenomenon, evaporation plays a fundamental role in the world we know. Droplets which contain nonvolatile solutes or particles leave a deposit once evaporated, which can be engineered to produce desired patterns and coatings (Kuang et al., 2014). Understanding the mechanisms behind these patterns and coatings is imperative in a variety of manufacturing applications including fabricating electronic devices (Kjellander et al., 2010), developing biological/chemical sensors (Abe et al., 2008), and applying uniform layers of paint in industrial applications (Curak et al., 2019). One widely researched deposition phenomenon is that of the coffee ring effect, in which during evaporation solutes are driven to the periphery of a droplet via capillary flow (Deegan et al., 1997). Further studies of the coffee ring effect have provided greater understanding of fluid flow at the micro-level and aided in designing mixtures and substrates which suppress the effect (Hu \& Larson, 2006).

Recently, another popular beverage has been found to form curious deposition patterns - American whiskey. Termed "whiskey webs", these self-assembled structures are a result of monolayer collapse as a diluted droplet of American whiskey evaporates. The web-like formations are unique to each product and generally repeatable when sampled from the same bottle of American whiskey. In a test of three separate whiskeys, samples 
were able to be matched to their "fingerprint" with an accuracy greater than $90 \%$ (Carrithers \& Brown, et al., 2020). It was also noted that web formations become less dense with increasing dilution, meaning that whiskey webs could potentially act as an identifier of adulterated products. Since whiskeys are so chemically complex, more research is necessary to determine the impact of individual chemical constituents and the possibility of using this method as a means of whiskey identification and/or quality control. It was suggested that identifying whiskey products based on their web formation could be improved using machine learning.

Machine learning, a subset of artificial intelligence, is a quickly growing technology used for performing predictive analytics. Essentially, one feeds large amounts of data to a computer and allows it to determine the optimal algorithms for a desired solution on its own. This technology is used in a variety of applications including selfdriving cars, personal assistants such as Siri, fraud detection for banks, resource scheduling, and safety systems (Mueller \& Massaron, 2016). Machine learning can be accomplished using a variety of algorithms. One such group of algorithms, neural networks, is exceptional for image pattern recognition. Properly training a computer to identify patterns requires a large database of images from which to learn. To use a neural network to identify American whiskeys based on their web patterns, thousands of whiskey webs must be produced and imaged for each sample. To this end, a means to produce a large database of imaged whiskey webs must be developed. 


\section{B. Purpose of Study}

The manual deposition and imaging techniques used in previous studies had low throughput (approximately 30 webs per hour), making acquisition of a database large enough for proper machine learning infeasible. The objective of this study was to develop a test apparatus to automate the fabrication and data collection of whiskey webs. The creation of such an apparatus will improve the testing consistency between researchers and be the foundation of large statistical studies of whiskey webs in the future. As described, the apparatus could produce and image 64 webs per hour without human intervention. Further optimization could result in hundreds of webs per hour. Constraints of the system were identified, and the dispensing technique was compared to the current method of manual deposition. 


\section{Significance of Study}

In Kentucky alone, the home of bourbon, the distilling industry creates an annual economic output of $\$ 8.6$ billion; with $\$ 452$ million of product shipped abroad in 2017 (Coomes \& Komstein, 2019). Operation OPSON is a Europol INTERPOL joint operation targeting fake and substandard food beverages on an international scale. In Operation OPSON VIII, which took place December 2018 through April 2019, illicit alcohol was the most seized item, totaling over 33,000 metric tons (Interpol, 2017). In addition to the economic impact, counterfeit/adulterated alcoholic beverages are a health concern as well. Bootleg spirits laced with methanol can be fatal. Considering the popularity of American whiskeys, it is economically desirable to identify and apprehend counterfeit products.

The testing apparatus described within will standardize the deposition technique for whiskey webs by eliminating human error from manual deposition. Improving the consistency of deposition methods reduces the variables which must be considered when comparing the web patterns between products. The improvement in consistency could enhance the accuracy of product identification and make possible more in-depth studies of the impact of various whiskey characteristics on their resultant web patterns, including the effects of aging and the addition of various chemical components. 


\section{CHAPTER II. LITERATURE REVIEW}

\section{$\underline{\text { A. Whiskey Webs }}$}

The following is an abbreviation of the work "Multiscale Self-Assembly of Distinctive Weblike Structures from Evaporated Drops of Dilute American Whiskeys" by Carrithers \& Brown, et al., published in the ACS Nano journal. Please refer to Appendix A for the full article.

A droplet containing nonvolatile solutes leaves a deposit whose form is dependent upon the intrinsic properties of the liquid (Poulard \& Damman, 2007; Sefiane et al., 2003), the nature of the solutes and particles (Anyfantakis et al., 2015; Yunker et al., 2011), and the environmental conditions under which evaporation takes place (Girard et al., 2008). A common evaporative phenomenon is that of the coffee ring effect, in which solutes concentrate and deposit along the perimeter of an evaporated droplet due to outward capillary flow replenishing the evaporated solvent at the pinned contact line (Deegan et al., 1997).

Suppression and utilization of the coffee ring effect has been an extensively researched topic due to its implications in coating and patterning processes (Mampallil \& Eral, 2018). The coffee ring effect has been studied in numerous liquids, including whiskey 
(Kim et al., 2016). Recently, diluted American whiskeys have demonstrated a new evaporative phenomenon in which monolayers of water-insoluble chemical components form self-assembled weblike structures upon evaporation. These structures have been termed whiskey webs. Figure 1 displays a selection of qualitatively repeatable patterns produced by evaporating 1.0 microliter droplets of various diluted American whiskeys onto a coverslip glass (S. Williams et al., 2019).

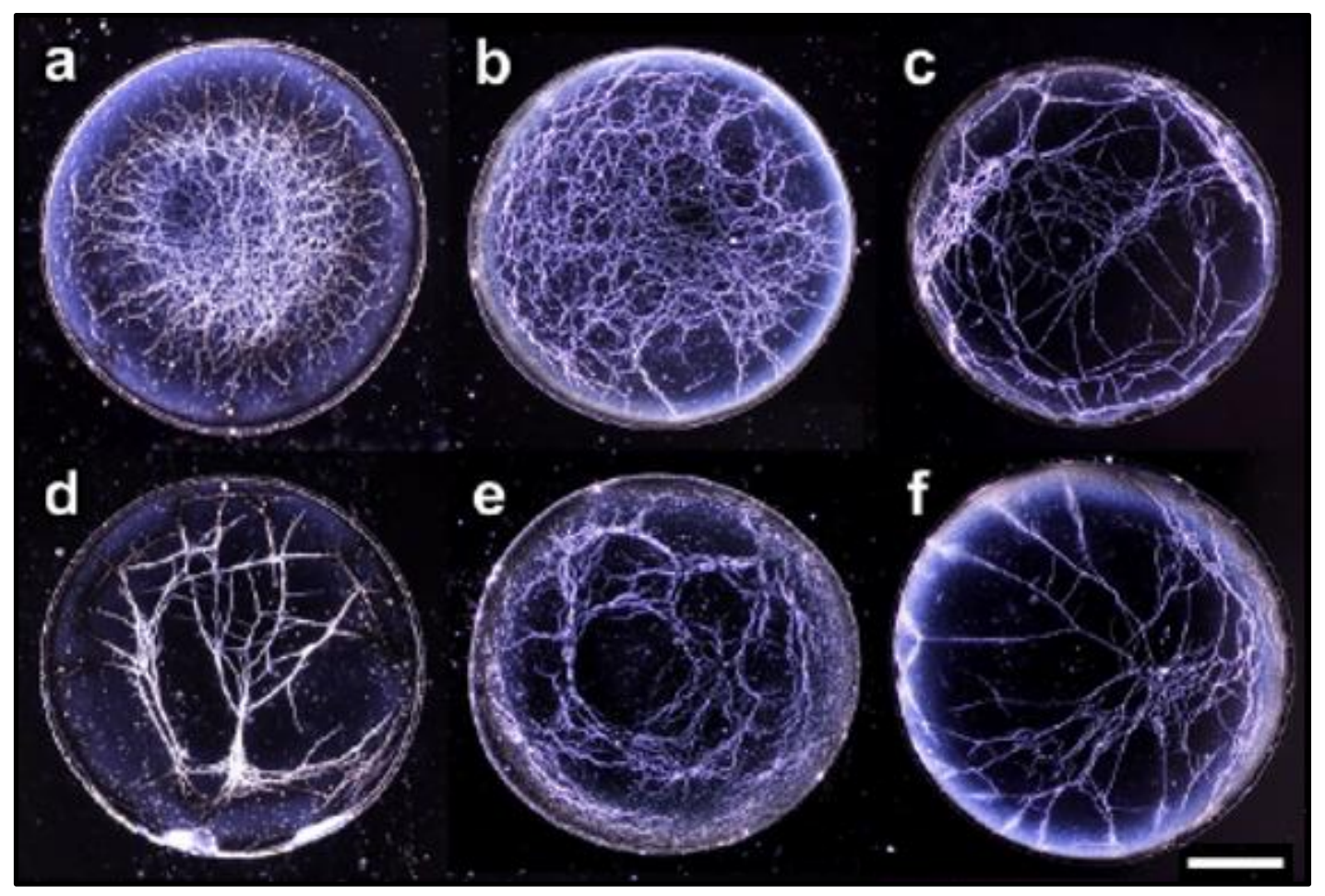

Figure 1. Whiskey webs formed with American whiskeys diluted to 20-25\% alcohol by volume: a) Old Rip Van Winkle 10 Year, b) Four Roses Single Barrel, c) Baker's Bourbon, d) Van Winkle Special Reserve 12 Year, e) O.K.I. 8 Year, f) Woodford Reserve Double Oaked. Scale bar is 0.5 millimeters. Modified from (Carrithers \& Brown, et al., 2020). 
American whiskey is a spirit distilled at less than $95 \%$ alcohol by volume (ABV) from a fermented mash of grain and is bottled at no less than 40\% ABV (Russell, 2003). Various forms of American whiskeys exist (i.e., bourbon whiskey, rye whiskey, wheat whiskey, etc.) with the defining characteristic being the mixture of grain used in the mashing process (Piggott et al., 1989). During fermentation, secondary products, known as congeners, are formed by side reactions and determine most of the organoleptic qualities of the final product. The congeners include phenols, aromatics, esters, aldehydes, higher alcohols, and trace substances (Bujake, 2007). After distillation, the product is typically matured in charred new oak barrels for at least two years. During the storage period, complex wood components are extracted by the liquid, resulting in an increase of acids, esters, and dissolved solids (Crampton \& Tolman, 1908; Liebmann \& Rosenblatt, 1943).

The variability in mash bill, fermentation conditions, and maturation between whiskey products contributes to the unique flavor profiles of each. These components can also be attributed to the uniqueness of the web patterns formed between different American whiskey products. To demonstrate this, a control bourbon whiskey was spiked with one of several key constituents that have been identified in whiskeys: acetic acid $(400 \mathrm{mg} / \mathrm{mL})$, ethyl laurate $(700 \mathrm{mg} / \mathrm{mL})$, lauric acid $(13.5 \mathrm{mg} / \mathrm{mL})$, and vanillin $(4.0 \mathrm{mg} / \mathrm{mL})$. These concentrations were selected to represent the mean level of each respective chemical or represent their collective chemical group (e.g., ethyl laurate for fatty acid ethyl esters), typically found in whiskeys (Freitas Jr., 2016). Figure 2 shows that each congener influences the structure of a formed whiskey web in a specific way. However, predicting how every congener within whiskey influences monolayer collapse is nontrivial. 


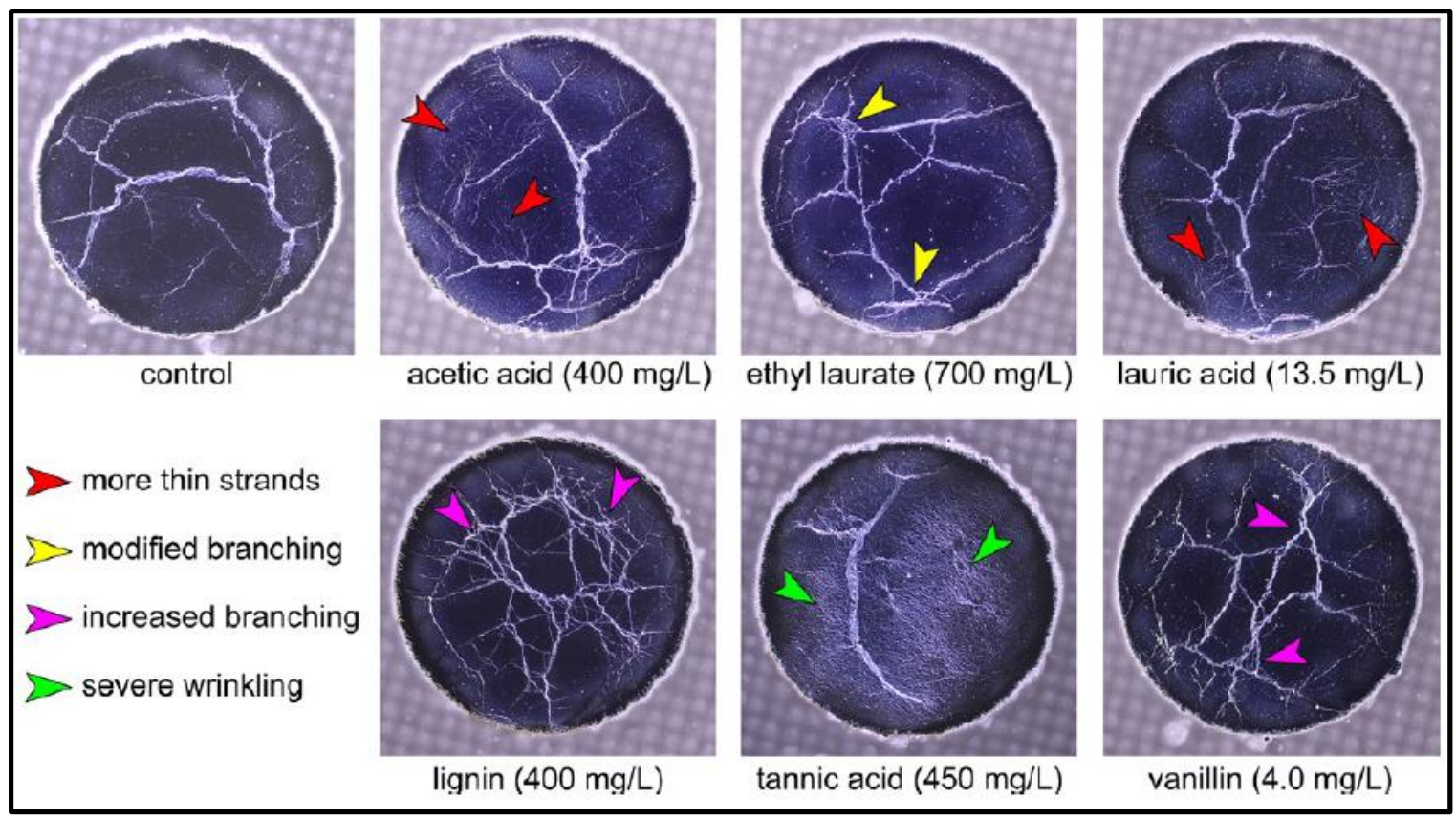

Figure 2. Effect of congeners on whiskey web patterns. Various congeners were added to a control bourbon whiskey to demonstrate that the concentration and combination of these compounds ultimately guide each whiskey pattern.

Modified from (Carrithers \& Brown, et al., 2020).

An aggregated image pattern identification test was conducted to evaluate the repeatability of evaporated whiskey web patterns and to demonstrate how digital image analysis could be used for identification, shown in Figure 3. Three different American whiskeys from the same distiller were selected, and 30 web images were acquired for each sample (90 total). Using a custom MATLAB program, each web image was converted to a binary image and masked so that only pixels inside a circle (with radius $R$ ) were analyzed. The pixels were blurred and placed into a bin based on radial position and pixel intensity (black was "0" and white was "1"). The resulting array was averaged for a randomly selected 25 droplets and used as the "fingerprint" for that sample. The remaining 15 images (five of each sample) were compared to the fingerprints of each of the three samples. The 
individual images were matched correctly to their sample over $90 \%$ of the time, thus demonstrating the repeatability of whiskey web formation.

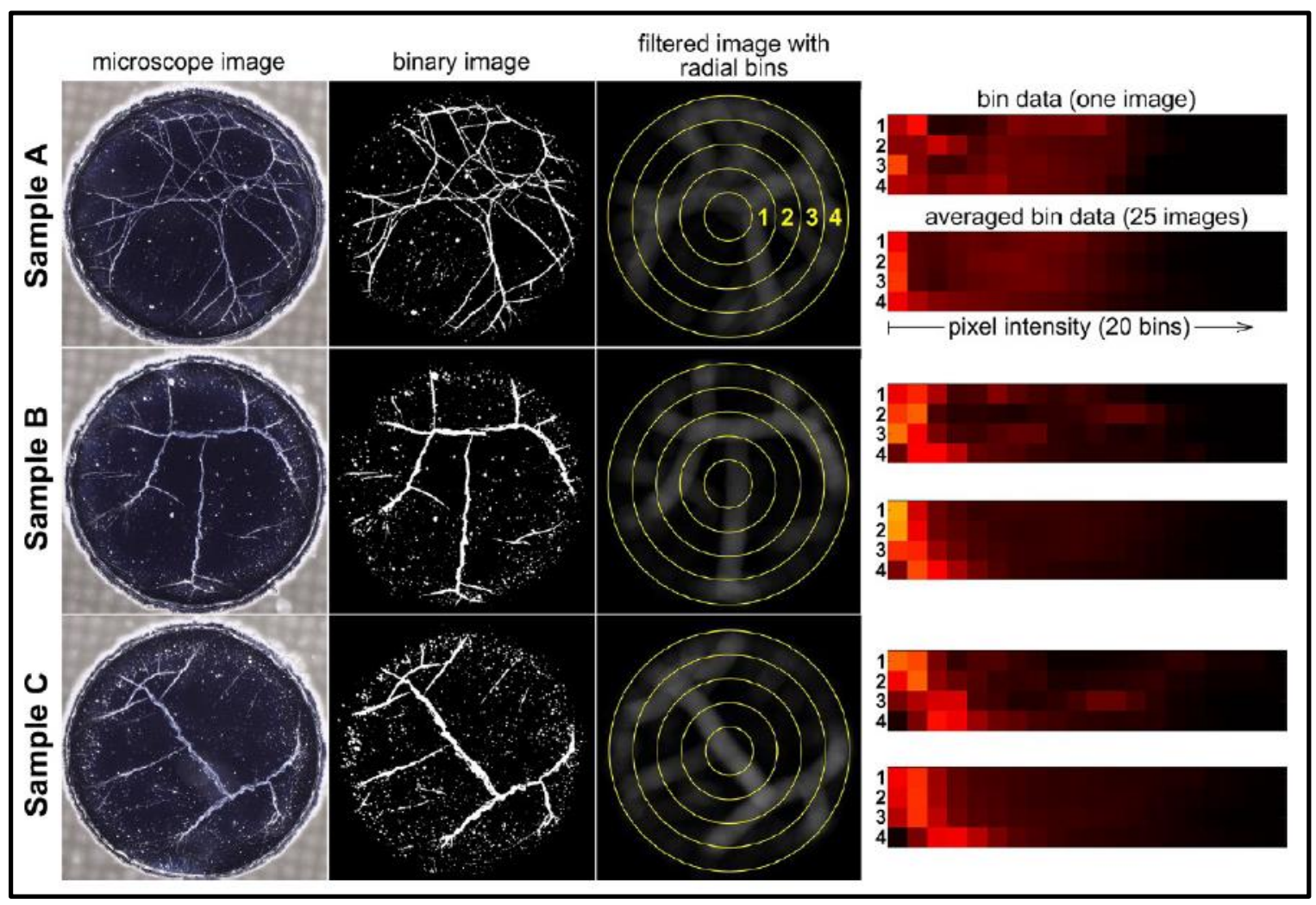

Figure 3. Analysis of microscope images of web patterns. Sample A) Old Weller Antique, B) W. L. Weller Special Reserve, C) W. L. Weller 12 Year.

Modified from (Carrithers \& Brown, et al., 2020).

This preliminary data shows that such digital image inspection may lead to simple chemical analysis of American whiskeys. Furthermore, this simple and surprisingly distinctive effect may be applicable to similar characterization of spirits (GonzálezGutiérrez et al., 2017) or other volatile liquids. 


\section{B. Image Pattern Recognition}

It was proposed that machine learning based image pattern recognition could enhance the identification of whiskeys based on their unique web patterns. Interest in pattern recognition and classification has grown significantly over the past decade due to the increased computational power of processors. Some applications for pattern recognition include data mining, biometrics, machine vision, speech recognition, selfdriving vehicles and medical imaging. Specifically related to the ophthalmological field, image pattern recognition is used in conjunction with retinal scans, such as the one in Figure 4, to assess more than 50 commonly diagnosed diseases (Liao et al., 2018). Whiskey webs share some resemblance to the veins found in retinal scans.

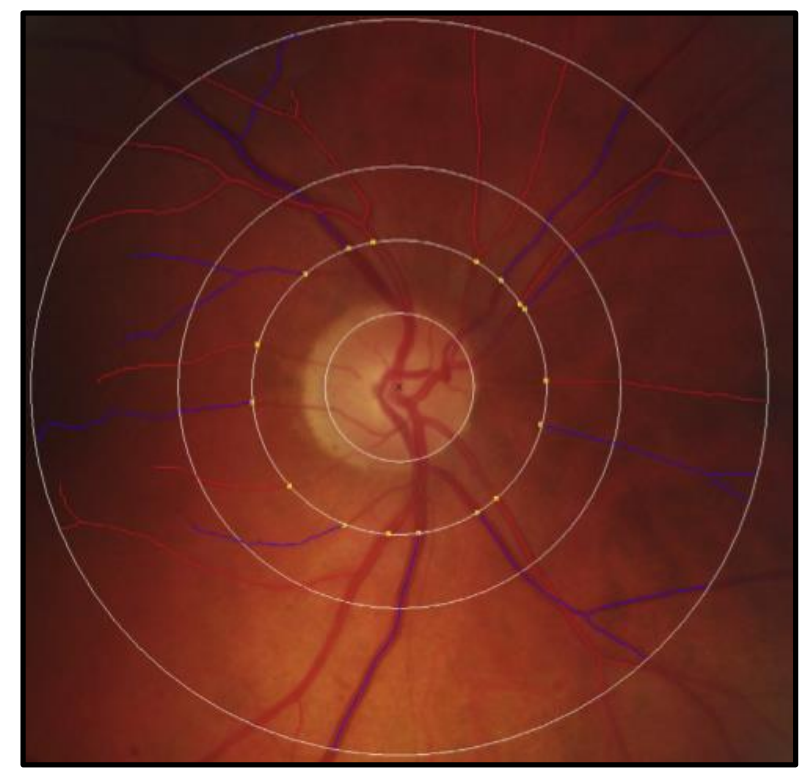

Figure 4. Retinal fundus image. Modified from (M. Williams et al., 2015). 
There are a multitude of classification methods used to train a computer to recognize patterns (Prince, 2012). Almost all machine learning can be interpreted in a probabilistic context; as such, results improve with larger datasets. A limited number of training data images may be insufficient for a neural network classification model to be trained properly. Solutions to limited data sets include transfer learning and data augmentation.

Transfer learning is realized by pretraining a model similar in domain to the desired application. The pretrained model's parameters are used as the initialization for the actual model which is then trained to solve the real problem with a smaller dataset. In the case of whiskey webs, representative models could be transferred from neural networks already trained to identify patterns similar to whiskey webs - such as the retinal fundus photography shown in Figure 4. With transfer learning, it is possible to teach a computer new classes in the same domain with as few as twenty samples.

Data augmentation takes a set of data and randomly crops, rotates, brightens, or warps the original image to increase the size of the training set. However, distorted images are not as desirable for overall accuracy, especially with minute details between classes.

It is, of course, desirable to have a dataset large enough that neural networks can be fully trained from the original dataset. Due to the subtle differences between certain whiskey web patterns, it is likely that thousands of images would be necessary to classify a single sample. 


\section{Thermal Inkjet Printing}

Inkjet technology has expanded beyond the office space and has gradually become a tool used in various industrial applications. Thermal inkjet (or bubblejet, as referred to by Canon) was invented independently by Hewlett-Packard Company and Canon in the late 1970s (Hammond, 1984). Thermal ink jet printheads are composed of three main components: a thin-film resistor (heater), a power-input controller, and an ink nozzle. The heater is activated by an electrical pulse of several microseconds and can have a heat flux of several megawatts per square meter. The heater quickly superheats a thin film of fluid which nucleates, forming a bubble that ejects the rest of the fluid from the nozzle (Chen et al., 1997).

The superheat limit "is approximately $90 \%$ of the fluid's critical temperature (i.e., the point beyond which a liquid phase no longer exists). The critical temperature depends solely on the physical properties of the fluid, whereas the superheat limit depends on ambient pressure and fluid properties." To eject a jetting fluid with repeatable velocity and volume, it is necessary for the heaters to homogeneously heat the fluid so that nucleation at the interface between the fluid and heater happens uniformly (Allen et al., 1985).

Viscosity and surface tension are the most crucial parameters in terms of a fluids jettability. The viscosity of the sample must be low enough to allow the channels to refill between successive fires of a nozzle. The surface tension of the sample must be high enough to prevent the sample from dripping from the nozzles (also called drooling). In general, thermal inkjet printers can be made to dispense fluids with a viscosity up to 100 centipoise, but typical inks are only 2 centipoise. The minimum surface tension is about 35 millinewtons/meter (Calvert, 2001). 
Today, inkjet technology is being used to develop devices such as chemical sensors (Abe et al., 2008) and electronic devices (Kjellander et al., 2010). Inkjet has become a portable technology that has unparalleled droplet deposition precision. While high-end electronic pipettes can only achieve volumes as small as 0.5 microliters, ink jetting can dispense droplets as small as 6 picoliters. Droplet volume accuracy and programmability makes ink jetting an attractive technology for microfluidic studies. 


\title{
CHAPTER III. MATERIALS AND METHODS
}

\begin{abstract}
$\underline{\text { A. Facilities }}$
Due to the onset of COVID-19, all fabrication and testing of the apparatus was done in the basement of my local residence. Due to issues with control of environmental conditions, tests were done in one of three locations: a large sized room ( 1000 square feet), a medium sized room ( 225 square feet), or a small sized room ( 70 square feet). Printhead analysis was conducted at Funai Lexington Technology Corporation.
\end{abstract}

\section{$\underline{\text { B. Device Fabrication }}$}

Custom parts for the apparatus were 3D printed using Polylactic acid (PLA) 1.75millimeter filament (Hatchbox). The parts 3D printed for the apparatus appear as red in computer generated images but are gray in the actual assembly. SolidWorks 2019-2020 Student Edition was used as the computer aided design software for modeling and rendering the apparatus. The 3D printed components were sliced using PrusaSlicer and fabricated using a PRUSA MK2S fused deposition modeling (FDM) printer. A full list of the $3 \mathrm{D}$ printed parts can be found in Appendix B. A detailed assembly of the apparatus can be found in Appendix C. 


\section{Device Design and Assembly}

\section{Concept Overview}

Two designs were evaluated as potential candidates for an automated whiskey web testing apparatus. Both prototypes used a developmental handheld thermal inkjet printer as a means of droplet deposition. The printer was provided by Funai Lexington Technology Corporation, and is referred to as the Fluidic Control Language (FCL) Controller, shown in Figure 5. The FCL Controller had two cartridge ports on each side of the device, allowing for simultaneous droplet deposition by four individual printheads. The FCL Controller was driven by the fluidic control language, which was provided as both an executable program and as Python code.

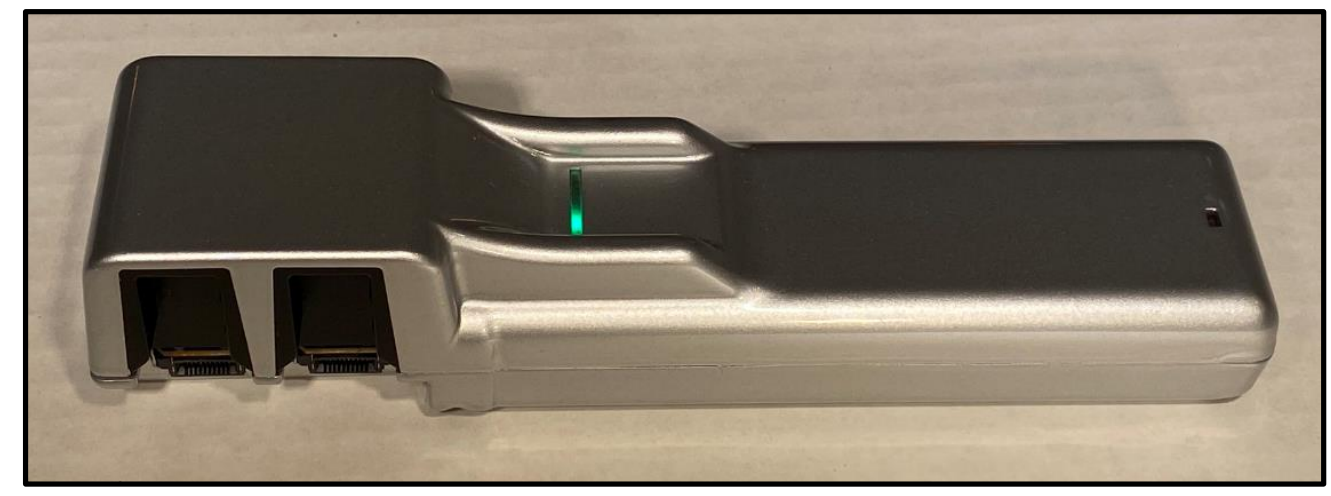

Figure 5. FCL Controller provided by Funai Lexington Technology Corporation.

In the first design, shown in Figure 6, the FCL Controller was mounted to two carriages which rode along guide rails in an $x-y$ plane. The carriages would be driven by stepper motors (not shown) to allow the FCL Controller to be maneuvered between bays where whiskey droplets were printed onto glass slides. 


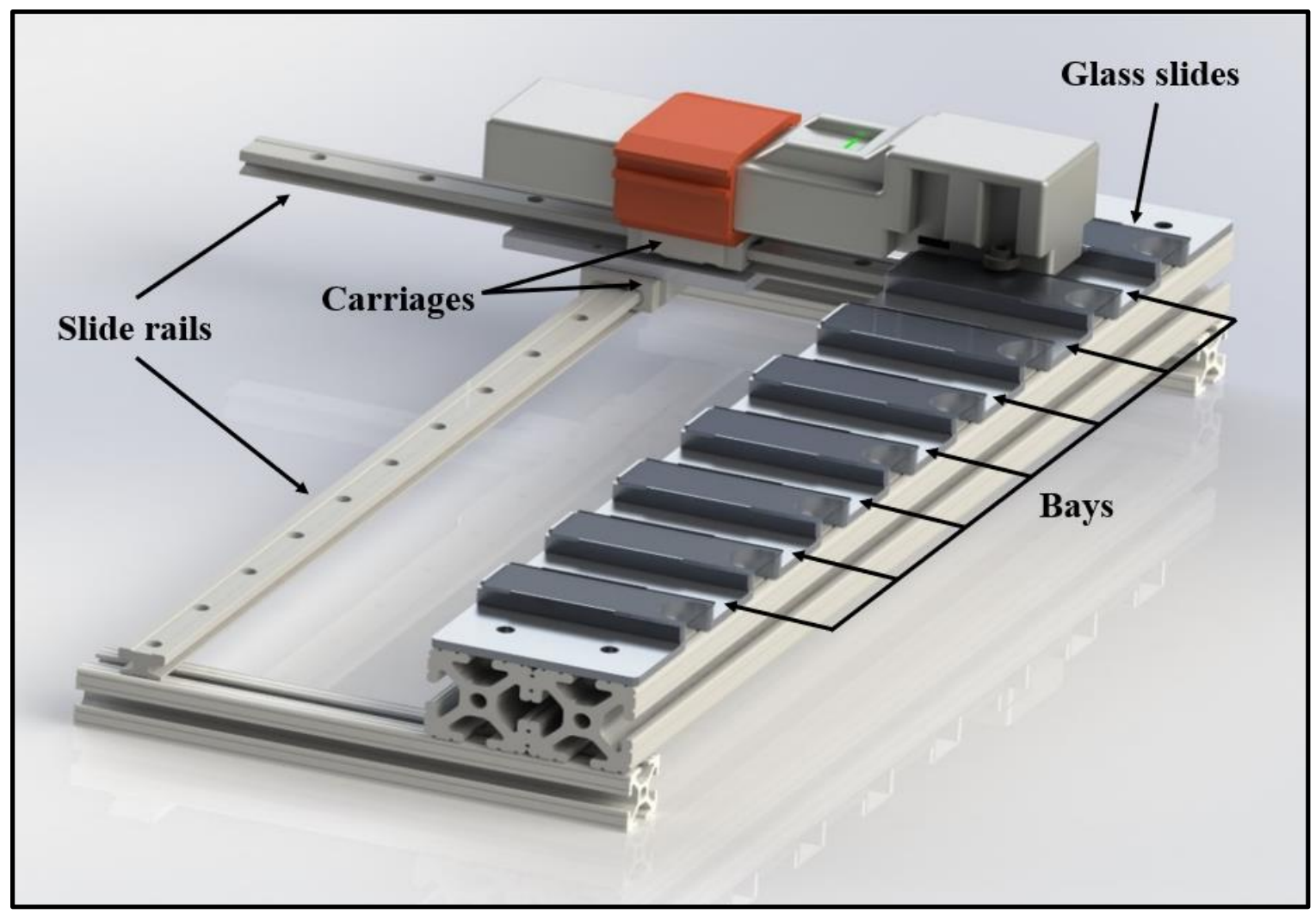

Figure 6. Rendered slide rail concept for semi-automation of whiskey web fabrication.

The design used few components, making it robust and easy to assemble. The printer moves in only a single plane, resembling a plotter, so the software to maneuver the printer would be trivial to code. The highest cost component of the concept was the aluminum tray on which the glass slides were nested, which as shown required the machining of an aluminum plate with the dimensions of $20 \times 3.0 \times 0.5$ inches. To take advantage of ports on both sides of the FCL Controller, the bays must be wide enough that the Controller could fit between them. Unfortunately, for the printheads to align with the glass slides the Controller only had 3/100ths of an inch between itself and each bay wall depicted in Figure 7. Any skew in the slide rails could have resulted in the FCL Controller ramming the bays and damaging the device. 


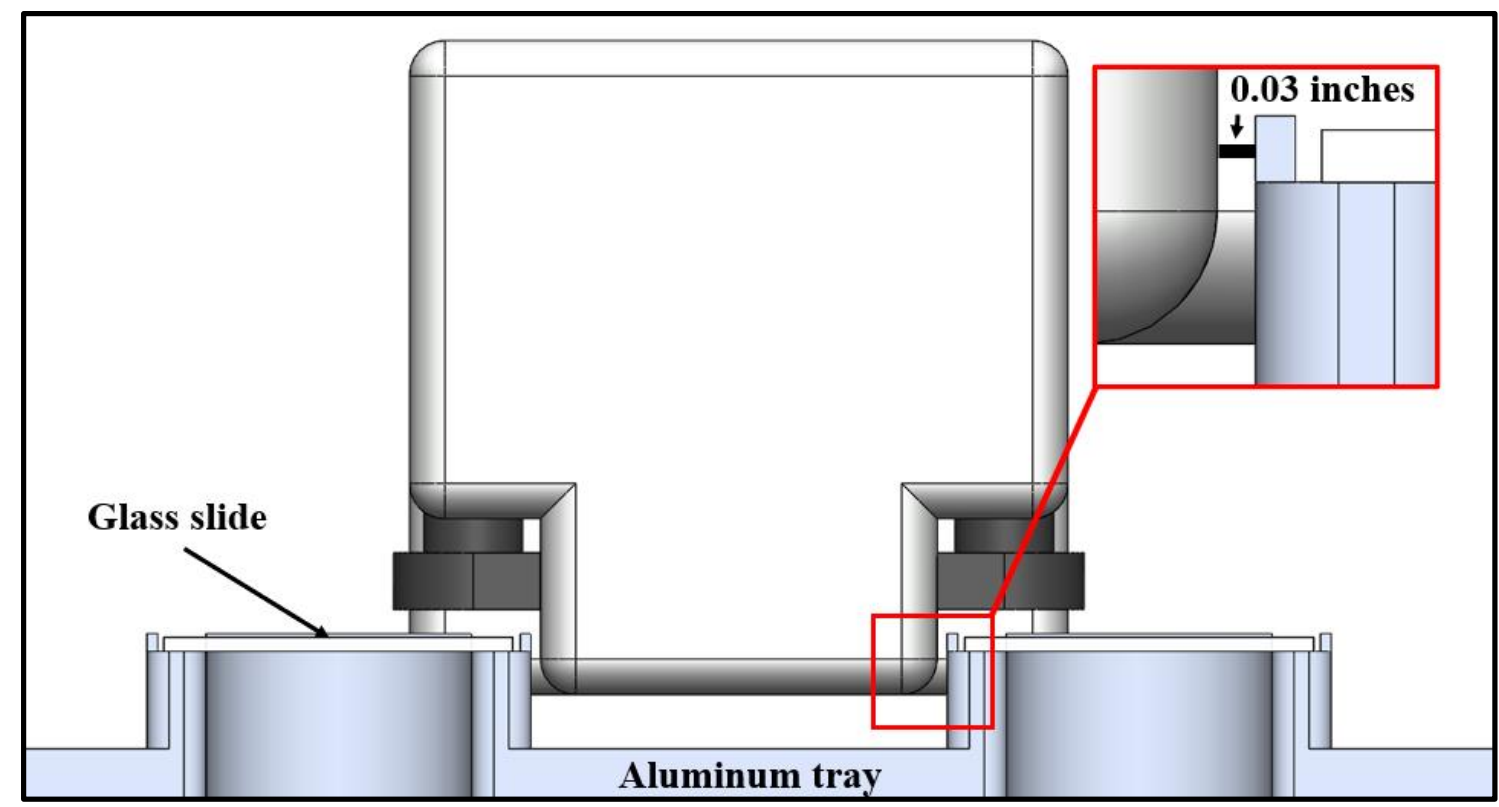

Figure 7. Front view of slide rail concept, depicting narrow gaps between walls of aluminum tray and FCL Controller.

Although simple and likely inexpensive to fabricate, the major drawback of the slide rail design was that it only semi-automated the necessary data collection. Firstly, the droplets were not automatically imaged after evaporating. Secondly, the glass slides must be refilled as the Controller progresses. Assuming a minimum distance of 4.0 millimeters between droplet centers, each glass slide could hold 32 droplets (the two outermost glass slides would only hold 16 droplets each). Therefore the slide rail concept depicted in Figure 6 could accommodate 224 droplets per test. Since machine learning can require thousands of images from a single class for accurate labelling, this design was determined to be inefficient.

A second design was devised as a means by which to fully automate the fabrication and imaging of whiskey webs; inspired by old-school film cameras. As shown in Figure 8, a reel-to-reel design used a pinch roller and capstan to pull a film beneath the FCL 
Controller printheads for droplet deposition, over a Digital Single Lens Reflex (DSLR) camera for imaging, and then onto an uptake reel. The reel of film was fifty feet long, so assuming the same 4.0 millimeter spacing between droplets, a single roll of film could test over 3500 droplets before needing to be replaced. The reel-to-reel design was certainly more complex than the slide rail design but allowed for full automation of data collection so that larger datasets could be produced with less human intervention. For this reason, the reel-to-reel concept was chosen for further development.

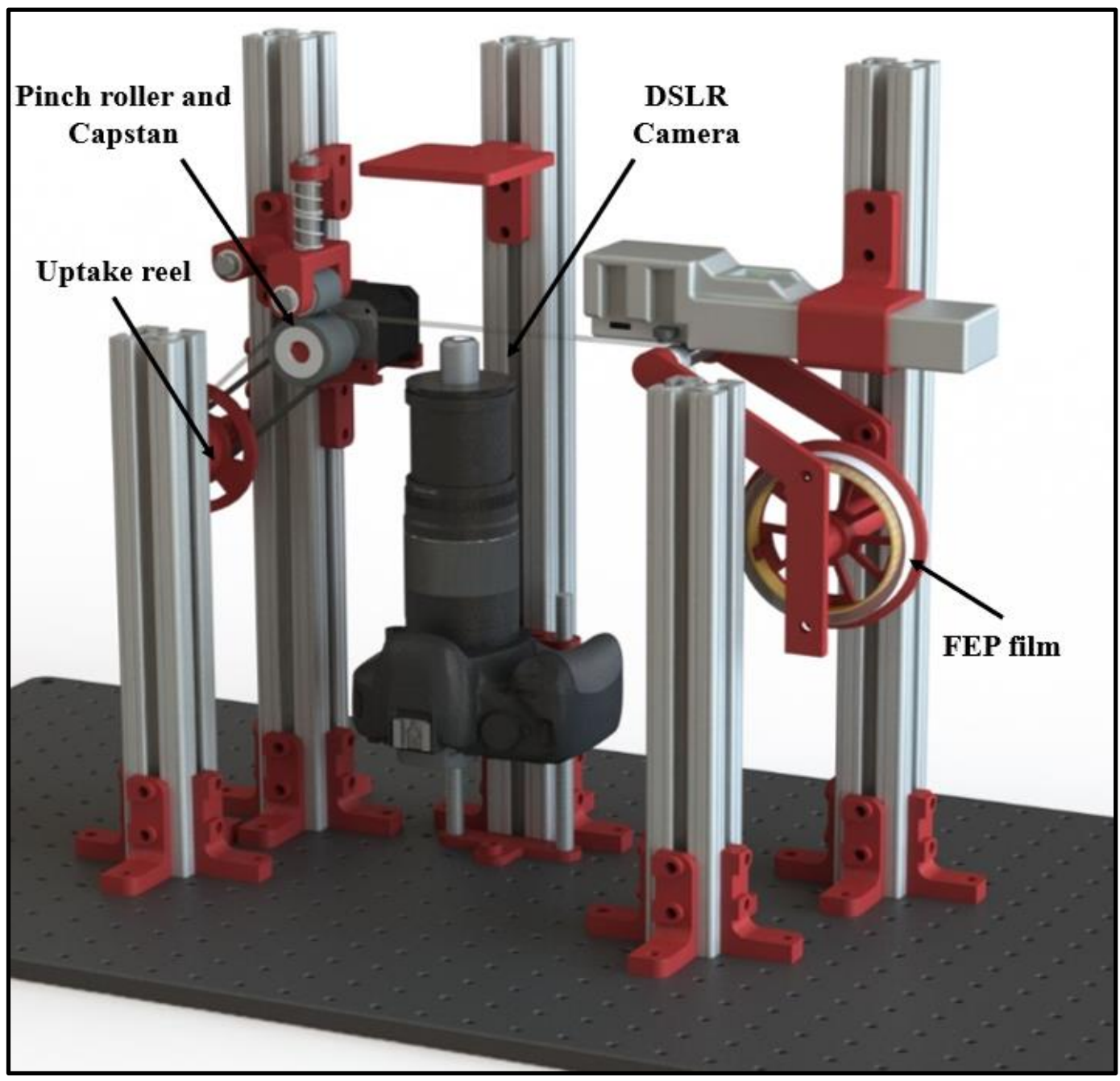

Figure 8. Rendered reel-to-reel concept for full automation of whiskey web fabrication and data collection. 
Design of the reel-to-reel apparatus (RRA) can be broken into four main subsystems: printing, tape transport, imaging, and electronics. The design of each subsystem is detailed below. Detailed assembly instruction can be found in Appendix C.

\section{Printing Subsystem}

A handheld development printer called the "FCL Controller" was provided by Funai Lexington Technology Corporation (FLTC). The Fluidic Control Language (FCL), instructions for use, and several empty printer cartridges were also provided. As shown in Figure 5, the printer had ports for two cartridges on each side of the device. While only one side of the device was used in the current design, it is conceivable that both sides of the device may be used to increase the rate of testing in future prototypes. It was recommended by FLTC that the printhead be positioned 1.5 millimeters above the substrate. A gap greater than 1.5 millimeters decreases the positional accuracy of dispensed droplets, resulting in irregularly shaped droplet profiles.

FLTC provided two variations of a single type of cartridge for testing. Visually,

one cartridge was short while the other was tall, as shown in Figure 9. The short cartridge did not have a filter between the reservoir and nozzles. The taller cartridge included a large body to increase the maximum volume, a stainless-steel mesh filter between the reservoir and nozzles, a felt filter, and a cap. The printhead chipset on each cartridge had 128 nozzles, broken into 8 primitives of 16 nozzles, each of which dispensed 24 picoliter droplets. 


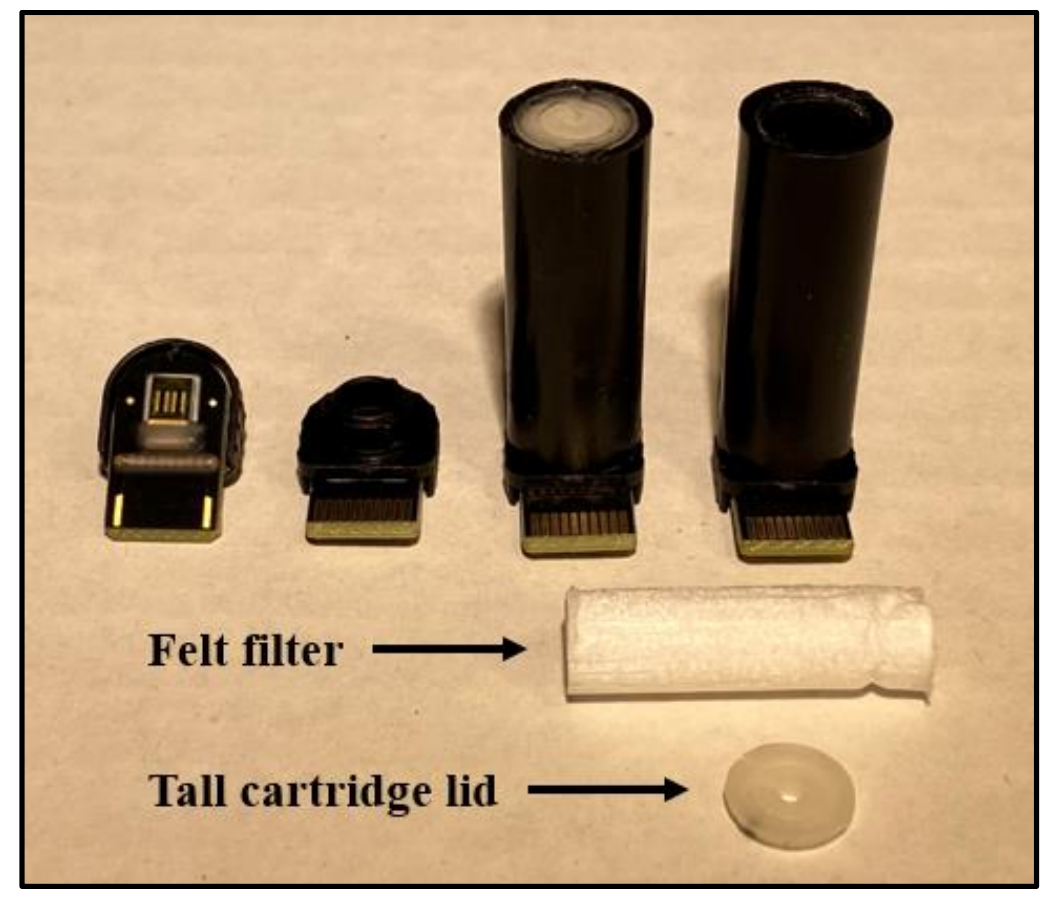

Figure 9. Short and tall printer cartridges provided by FLTC.

Rightmost cartridge has felt filter removed.

The FCL allowed editing of several printing parameters, the most important of which are shown in Table 1. The dose was the amount of fluid (defined in microliters) that was dispensed for a single run command. The preheat temperature was the temperature to which the entire chipset was heated before printing. The preheat temperature decreased the thermal delta between the sample fluid and its nucleation temperature. Previous studies showed whiskey webs do not form when the sample is subjected to high temperatures, therefore the preheat temperature was set to its lowest recommended value of $40^{\circ} \mathrm{C}$.

The design of the cartridge chipset allowed for the programming of individual nozzles to jet fluid. Figure 10 shows a simplified mockup of the chipset and how nozzles 
were addressed. Each chipset had a total of 128 nozzles which were divided equally between eight zones referred to as primitives $(\mathrm{P})$. Each nozzle in a primitive was further defined by a specific address (A), one through sixteen, so that individual nozzles could be chosen for dispensing. The incremental address determined the number of addresses to increment between fires. For example, an incremental address of 3 means every third nozzle in a primitive will be fired in a loop until the total volume of fluid is dispensed. An incremental address of 16 means only one nozzle will fire for the duration of the dispense.

The remaining parameters given in Table 1 must be designed for each liquid to ensure optimal dispensing. Although not optimized for whiskeys, FLTC was able to provide settings for the frequency, voltage, and fire pulses for a general solution containing $25 \%$ alcohol by volume. The frequency is primarily dependent on the viscosity of the fluid, as the nozzle chambers need time to refill between successive fires. The voltage, pre-fire, delay, and main fire are all a function of the nucleation temperature of a fluid.

Table 1. Printing parameters for FCL Controller.

\begin{tabular}{|l|c|c|}
\hline Printer Parameter & Default & Recommended \\
\hline Dose $(\mu \mathrm{L})$ & 1.0 & 1.0 \\
\hline Preheat temp $\left({ }^{\circ} \mathrm{C}\right)$ & 45 & 40 \\
\hline Primitive & $1-8$ & $1-8$ \\
\hline Address & 1 & $1-16$ \\
\hline Incremental Address & 3 & 16 \\
\hline Frequency $(\mathrm{Hz})$ & 1000 & 3000 \\
\hline Voltage $(\mathrm{V})$ & 9 & 10 \\
\hline Pre-fire $(\mathrm{ns})$ & 100 & 150 \\
\hline Delay $(\mathrm{ns})$ & 300 & 1000 \\
\hline Main fire $(\mathrm{ns})$ & 800 & 660 \\
\hline
\end{tabular}




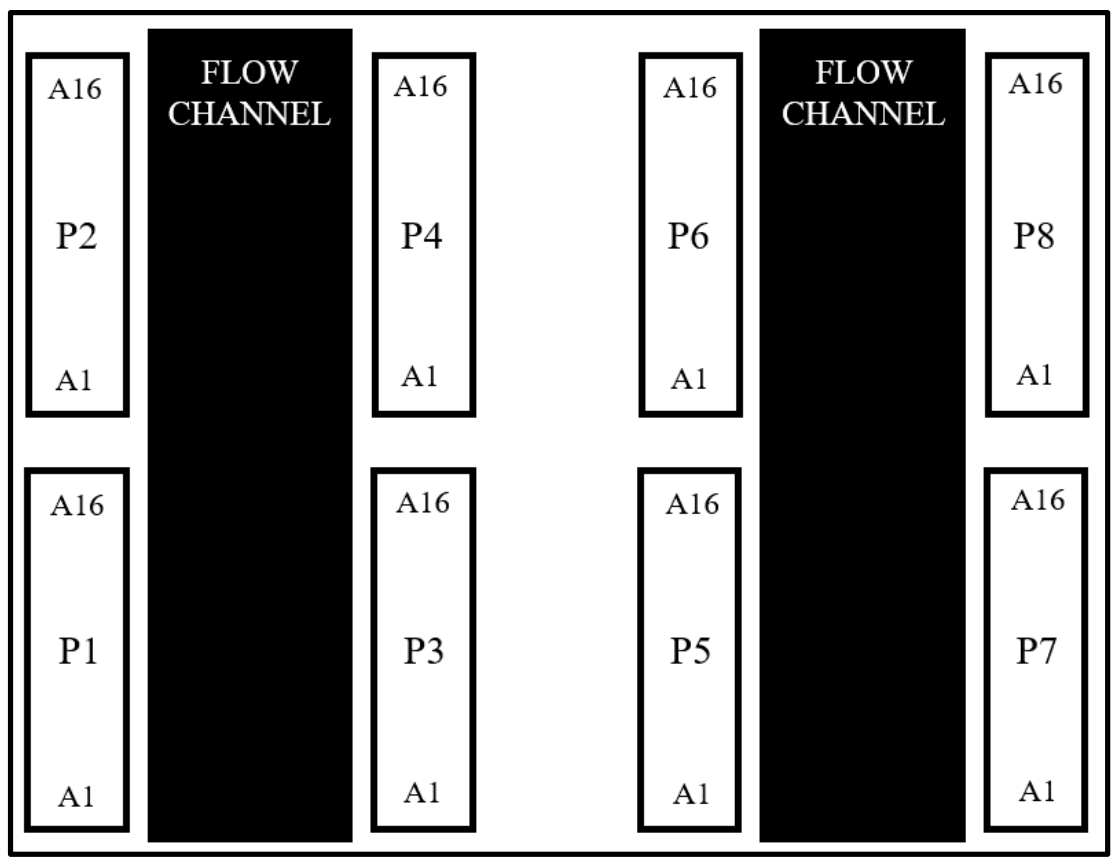

Figure 10. Mockup of nozzle placement on printer cartridge provided by FLTC.

\section{Tape Transport Subsystem}

In previous work (Carrithers \& Brown et al., 2020), glass slides with polytetrafluoroethylene (PTFE) printed wells were chosen as the substrate for testing. The PTFE walls of the wells provided a hydrophobic barrier to ensure a consistent profile of the droplets. In selection of a film for the transport system of the RRA, optical transparency and hydrophobicity were the greatest considerations. Fluorinated Ethylene Propylene (FEP) is another fluoropolymer which is similar in structure to PTFE and has similar material properties. In addition to hydrophobicity and optical transparency, FEP is available as a thin film, making it an excellent candidate for the RRA. A 50-foot roll of FEP film 0.5 inches wide and 5 mils thick was used for testing. The film was purchased from CS Hyde Company, with a special request for the roll to be air-cut. The standard 
cutting method for the film involved a cutting oil which left a residue on the outer edges of the film that negatively impacted the formation of whiskey webs.

The assembly was designed around the premise of transporting droplets from a droplet dispensing system to an imaging system. Film must be pulled from a supply reel, directed through the apparatus, and finally wound onto an uptake reel. Initially the concept used a stepper motor to drive the uptake reel itself. However, the effective radius of the uptake reel would increase as film accumulated, thereby varying the distance the film was pulled with each step and complicating the alignment of droplets with the camera lens. The solution to this issue was to use a capstan and pinch roller, as commonly found in cassette tape players, discussed below. A front view of the proposed tape transport system using a capstan and pinch roller is shown in Figure 11.

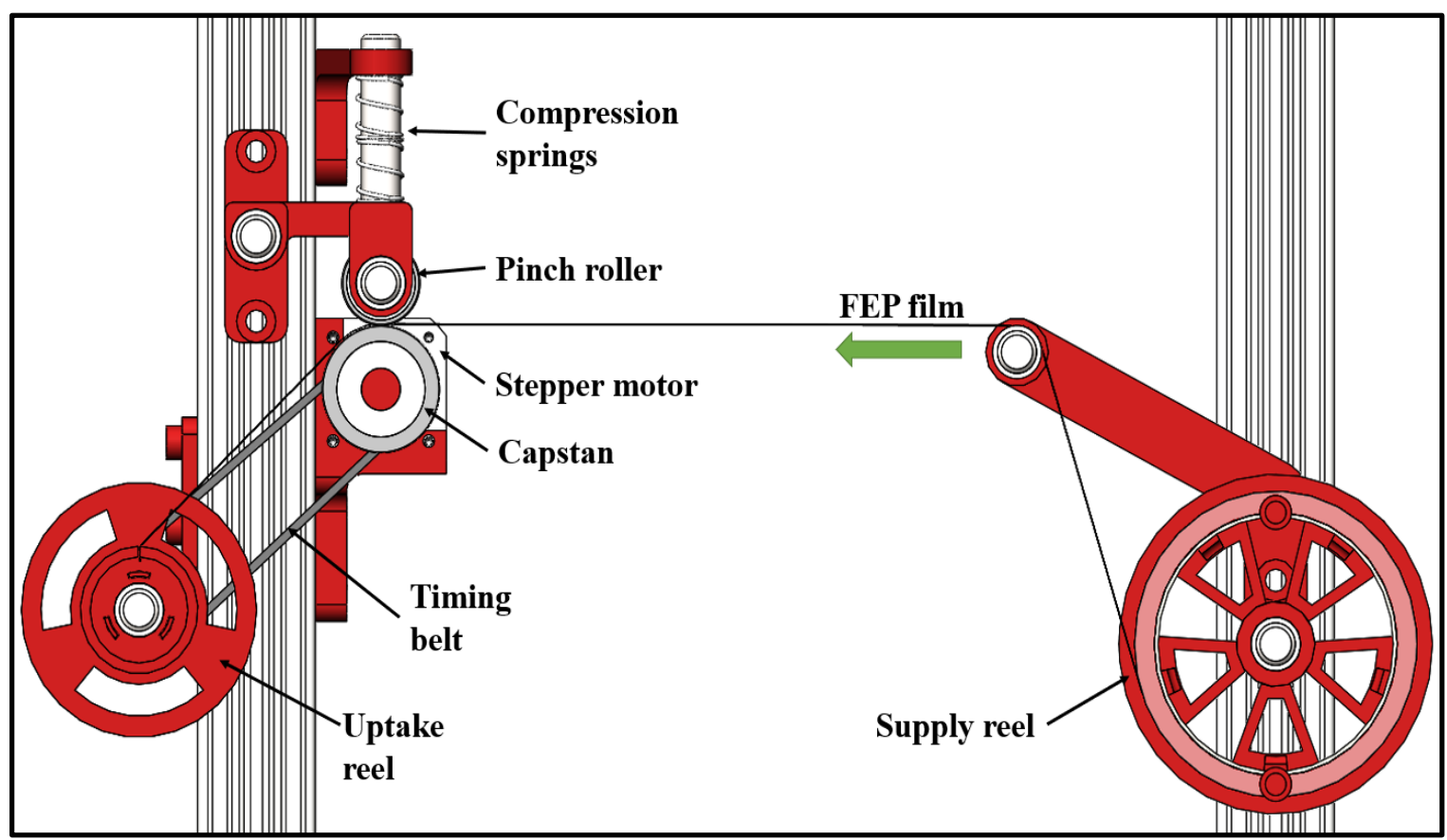

Figure 11. Front view of tape transport subsystem. 
The capstan was placed downstream of the supply reel (in direction of film motion) and was driven by a hybrid stepper motor. A stepper motor was chosen as opposed to a DC motor to allow for precise control of where each droplet was printed on the film, aiding in alignment with the camera. To ensure the film did not slip when the capstan turned, a normal force was applied to the film by a pinch roller which was held downward by compression springs. Once the film moved beyond the capstan it was wound onto an uptake reel which was also driven by the motor via a timing belt. The effective diameter of the uptake reel was equal to that of the capstan and was driven at a ratio of 15:17 to ensure the film was wound loosely, avoiding excessive downstream tension.

To maintain tension on the upstream side of the capstan, brakes were mounted against the supply reel, inducing drag. Figure 12 shows a close-up of the final prototype, displaying 10-millimeter diameter self-adhesive rubber bumpers adhered to 3D printed caps (supply reel brakes) which applied pressure to the FEP supply reel. The amount of the drag could be varied by adjusting the two bolts which were fastened to t-slotted framing, end-feed single nuts. The rubber bumper brakes were inset towards the center of the reelensuring pressure was maintained on the inner ring even as the supply of film dwindled. 


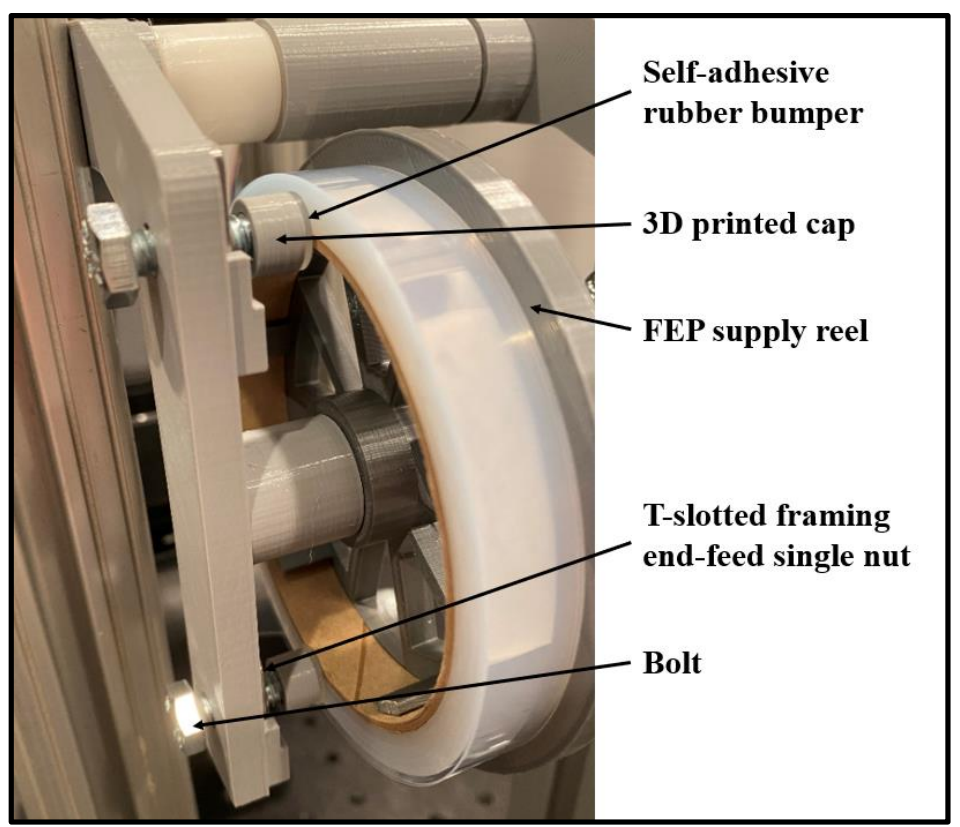

Figure 12. Close-up of prototype displaying two brakes inducing drag on the supply reel.

To compensate for misalignment, which may be inherent in a 3D printed system due to thermal warpage, it was desired the width of the capstan be greater than the width of the film. A neoprene driver roller with a 1.5-inch diameter and width of 1.0-inch acted as the capstan. The pinch roller was also one inch wide, but only 1.0 -inch in diameter. To reduce friction of the spinning parts, light duty dry-running nylon sleeve bearings were inserted into each part before being mounted to a 0.5 -inch diameter rotary shaft.

The parts in the tape transport system were oversized and could be made smaller in future prototypes. The neoprene drive roller was the major contributing factor to the size of the components - the smallest available 1-inch wide roller had a shaft diameter of 0.5 inches. To minimize the bill of materials, the 0.5 -inch diameter rotary shaft was used throughout the rest of the design, affecting the size of the pinch roller, sleeve bearings, compression springs, and various 3D printed components. 


\section{Imaging Subsystem}

In previous studies, whiskey webs were imaged using an inverted microscope (Nikon Ti-U). Since it was not practical to build the RRA apparatus around a large standalone microscope, the entire system was designed to mount directly to an optical bread board. As shown in Figure 13, the camera and ring light were mounted to a single piece of t-slotted framing rail which was mounted to the optical breadboard. The camera was mounted by attaching it to a standard square tripod mount which was then inserted into a 3D printed camera mount adapter. The adapter tightly slid into the t-slotted framing rail. Thru-holes were symmetrically located on each side of the adapter so that threaded rods could pass through the adapter and act as lead screws for coarsely adjusting the height of the camera.

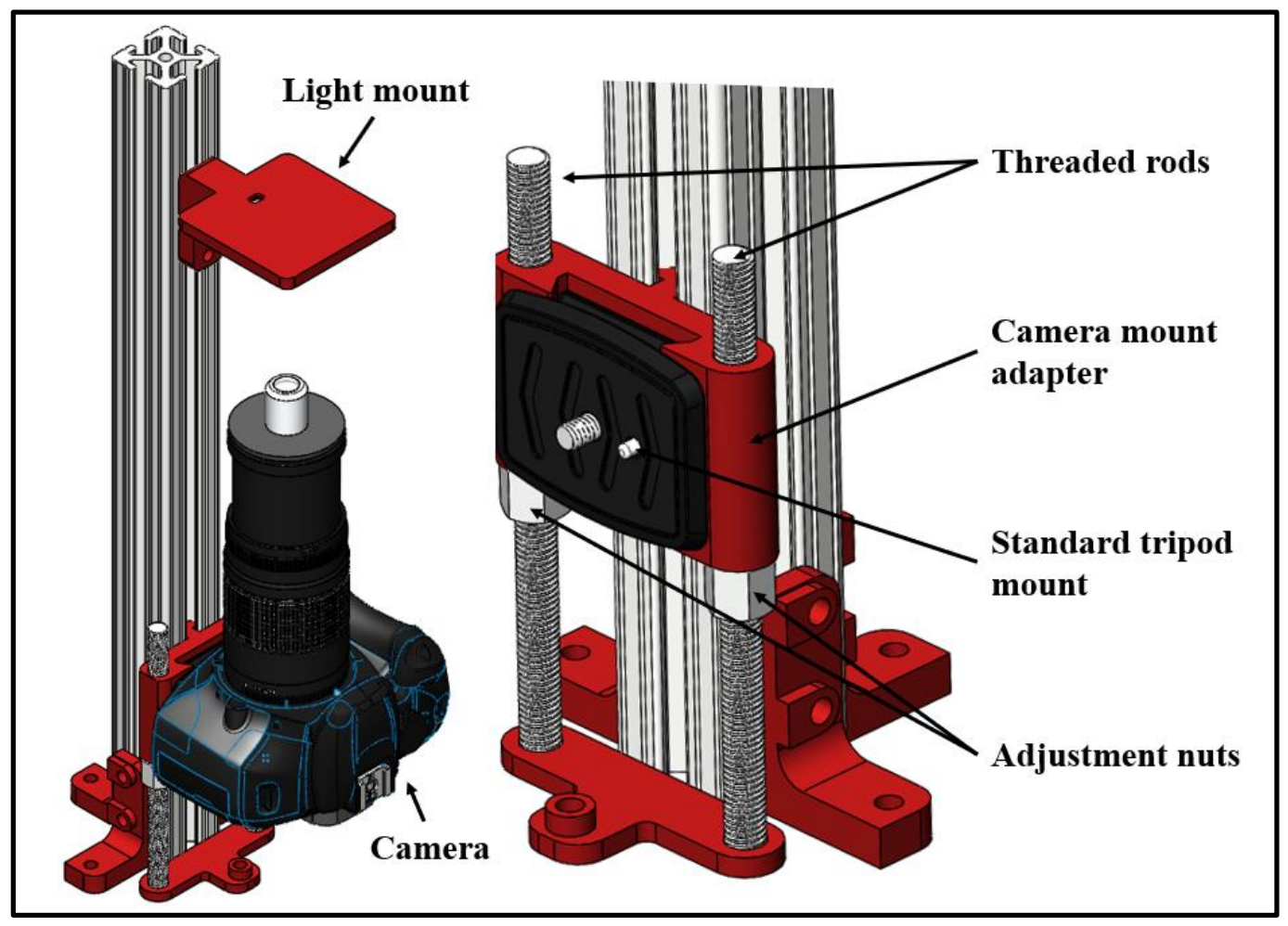

Figure 13. Imaging subsystem assembly. 
For proper magnification of the evaporated droplets, it was necessary to mount the objective lens to a camera lens with an appropriate focal length. The mechanical tube length of an optical microscope is the distance between the shoulder of the objective and where the image is being viewed. Today, tube length is standardized to 160 millimeters for finitecorrected transmitted light microscopes by the Royal Microscopical Society (RMS) typically denoted on the side of the objective (Abramowitz \& Davidson, 2020). The parfocal length, the distance from the shoulder of an objective to the focal plane, is also useful when attempting to focus the lens (Davison, 2020). For the RRA, an American Optical 4x infinity-corrected objective lens was mounted to a 55-250 mm telescopic lens. Since not otherwise noted, it was assumed that the focal length of the objective was standard. A Canon EOS 80D DSLR camera was used for imaging. The image sensor dimensions of a Canon EOS 80D are 22.5 x 15 millimeters and the image sensor pixel size is 3.7 micrometers.

\section{$\underline{\text { 5. Electronics Subsystem }}$}

Full operation of the RRA requires the following electronic functions: droplet deposition, stepper motor movement, imaging, illumination, and temperature/humidity sensing. Python, an open source object-oriented programming language, was used as the primary coding language for programming the hardware and properly sequencing the system events. Except for the FCL Controller, each electrical subsystem function was executed through operation of an Arduino Mega 2560. An Arduino is a microcontroller many hobbyists and DIY enthusiasts use due to its simple user interface and superb 
software support. Arduino uses a modified version of the $\mathrm{C}$ programming language and has its own Integrated Development Environment (IDE). The components operated via the Arduino are described below. Electronic components which could not interface directly with a breadboard were soldered using a lead-free soldering kit (Weller Electric) and insulated using liquid electrical tape (Performix).

\section{i. Stepper Motor}

A two-phase hybrid stepper motor (1.8 degree full-step) with 83.6 inch-ounces of torque was used for testing. A motor driver capable of excitation modes of 1, 2, 8, and 16 was used as the interface between the Arduino and stepper motor. Excitation modes allow stepper motors to be driven in step increments of less than one; with an excitation mode of 2, 8, or 16 corresponding to half stepping, eighth stepping, or sixteenth stepping, respectively. The motor used in this design was rated at 2 Amps. An Arduino Mega 2560 based on the Atmega2560 chipset has a recommended maximum output of 20 milliamp per input/output (I/O) pin. A motor driver and external 2 Amp power supply were used to power the motor.

A concern with stepper motors is the amount of heat generated while stationary. To precisely maintain their position, stepper motors always pull maximum current. It is not uncommon for stepper motors to reach temperatures of $100-110^{\circ} \mathrm{C}$. In anticipation of overheating, the 3D printed motor mount was designed so that the body of the motor abutted the aluminum framing rail to act as a heat sink, as shown in Figure 14. It was found during testing that the internal resistance of the stepper motor was great enough to maintain 
the tension in the film even when the stepper motor was off. Turning the motor off between droplets helped conserve energy and reduce the amount of heat generated. The motor's specification sheet can be found in Appendix D.

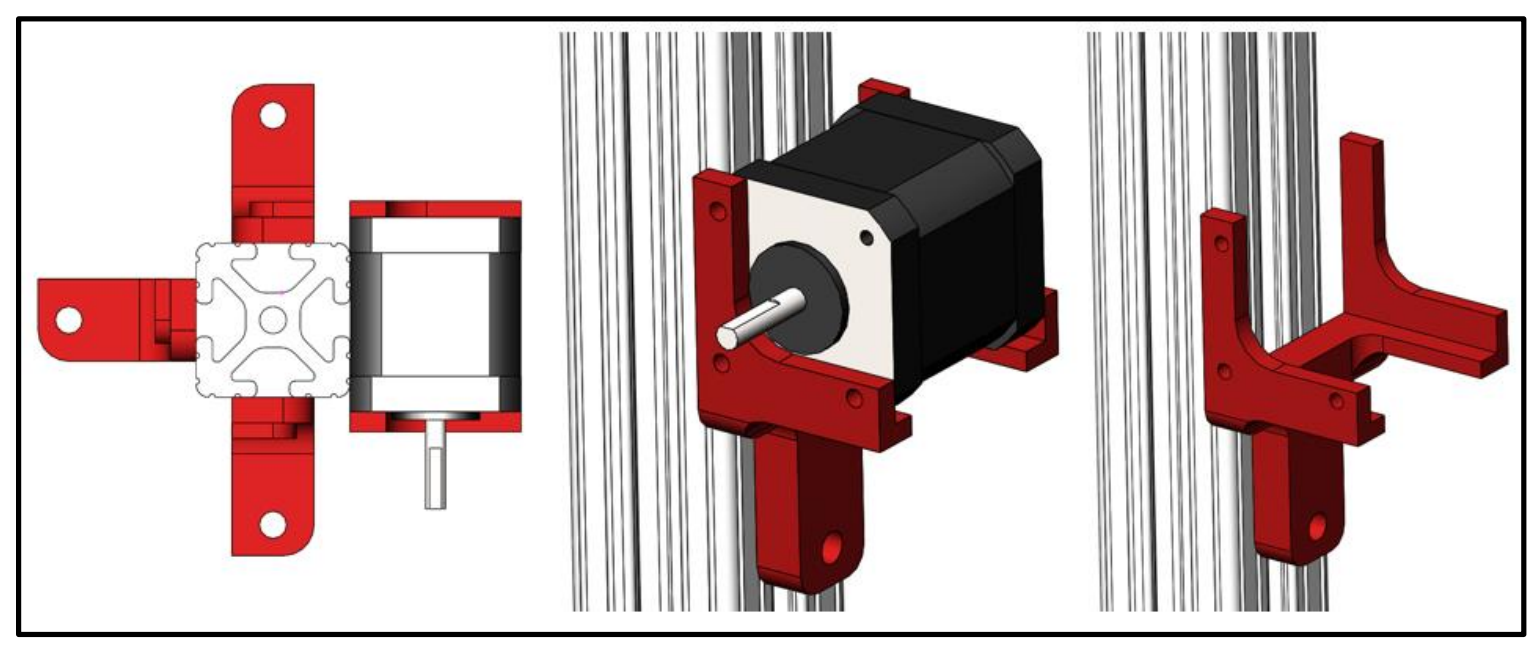

Figure 14. Motor mount designed to provide conductive heat transfer between aluminum mount and motor body.

\section{ii. Imaging}

As stated, a Canon EOS 80D was used to capture images of the droplets once evaporated. Canon's EOS Utility software was used to aid in focusing of the lens and for manually triggering the camera's shutter for remote shooting. To automate the imaging of the system, it was necessary for the camera shutter to be triggered by the Arduino via an intervalometer. An intervalometer functions by completing a circuit within the camera which triggers the shutter to open and close. The circuit is completed using a 2.5 -millimeter Tip-Ring-Sleeve (TRS) plug. As shown in Figure 15, the tip of the TRS plug corresponds 
to the camera's shutter release, the ring corresponds to the camera's autofocus, and the sleeve corresponds to the ground/common.

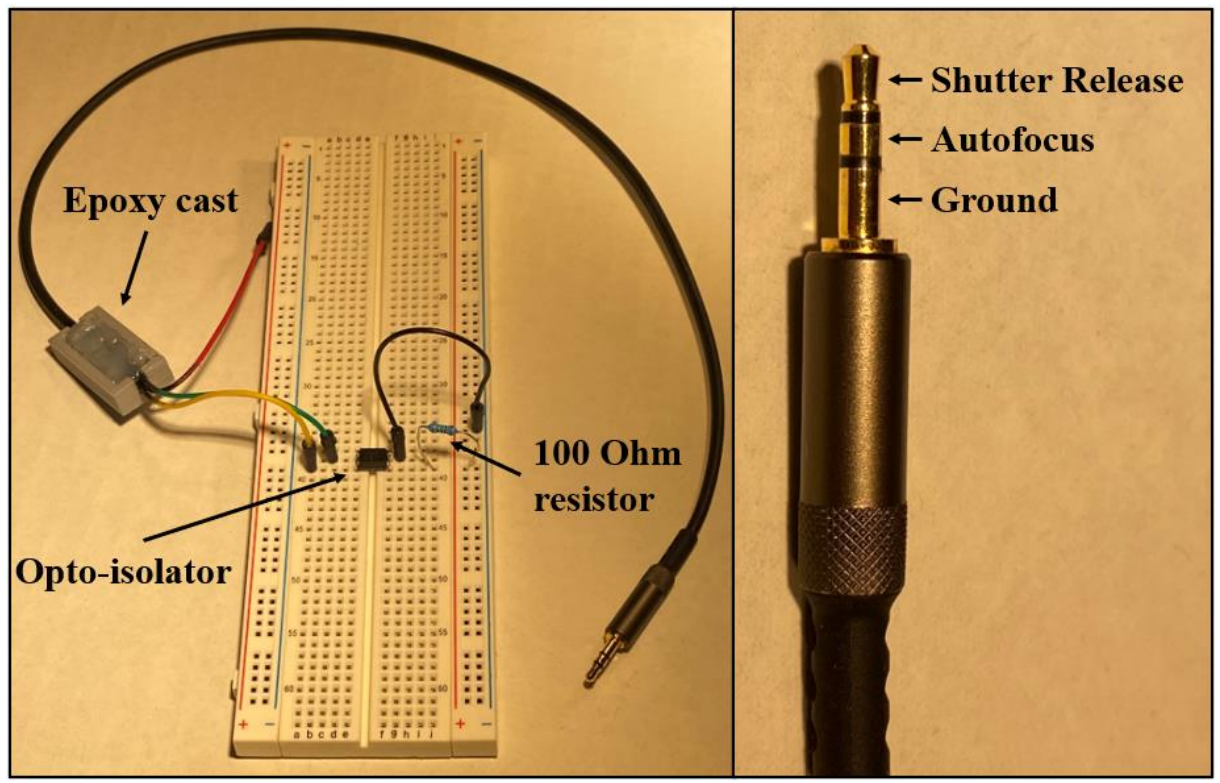

Figure 15. (Left) Do-it-yourself intervalometer prepared for connection to Arduino.

(Right) Pinout for the 2.5-millimeter TRS plug.

For this application, one end of a 2.5-millimeter TRS plug cord was stripped and carefully soldered to male-end jumper wires. The TRS wires were fragile, so each solder was covered in liquid electrical tape and then placed in an epoxy cast for protection. The green wire corresponded to the sleeve of the TRS plug (ground), the red wire corresponded to the ring (auto-focus), and the yellow wire corresponded to the tip (shutter release). Since microscope objectives do not have auto-focus capabilities, the red wire was unused.

To protect the camera's sensitive electronics from the Arduino, an opto-isolator (PC817) was used to isolate the electrical systems. An opto-isolator works much like a relay but uses a LED and phototransistor to complete an adjacent circuit instead of a mechanical switch. A 100 Ohm resistor was used to protect the opto-isolator from being 
overpowered. Not shown in Figure 15, the positive power rail was connected to an Arduino logic pin, and the negative power rail was connected to the Arduino's ground pin.

\section{iii. Illumination}

A ring light was made by adhering LED strip lights to the bottom of the 3D printed light mount, as shown in Figure 16. The LED strip lights were cut into sections three diodes in length, placed along the sides of the light mount, and soldered together at the terminals. Specifications for the LED light strip were unavailable. It was presumed that the LEDs would require a power output greater than what could be supplied by the Arduino. A relay was used so that the lights could be powered from the motor's 2-amp power supply while still being controlled by the Arduino.

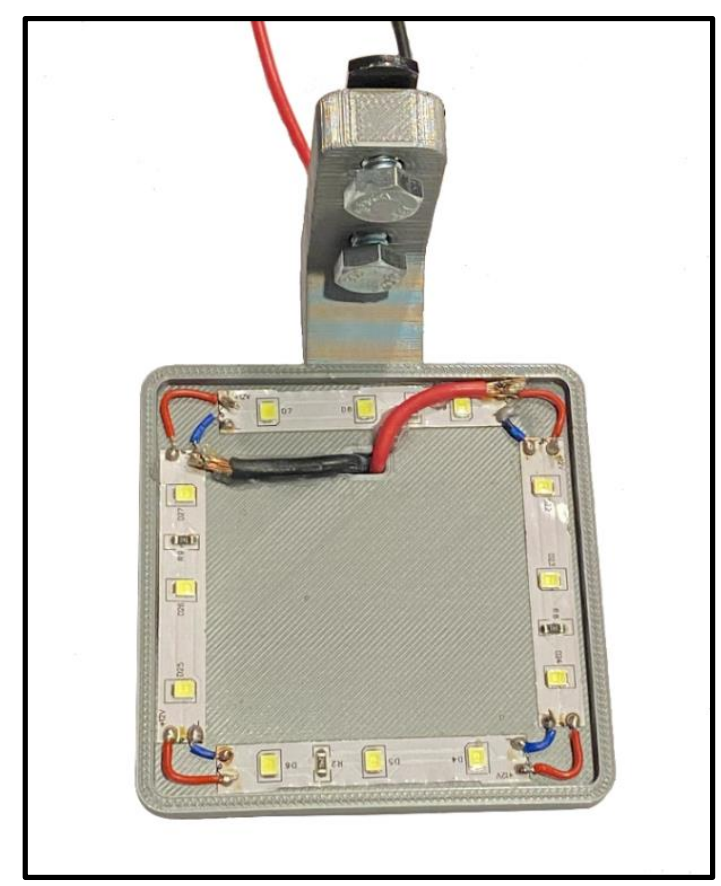

Figure 16. DIY light ring made by adhering LED strips to a 3D printed holder and soldering terminals together. 


\section{iv. Temperature and Humidity Sensing}

Previous studies indicated that temperature and humidity play a critical role in the formation of whiskey web patterns (Carrithers et al., 2018). Temperature and humidity affected the rate of evaporation of the droplets, therefore changing the fluid mechanics and monolayer collapse mechanisms which result in the web formations. A DHT22 digital temperature and humidity sensor was selected to record environmental conditions during testing. The DHT22 used an AOSONG AM2302 chipset capable of measuring a temperature range of $-40{ }^{\circ} \mathrm{C}$ to $80{ }^{\circ} \mathrm{C}\left( \pm 0.5^{\circ} \mathrm{C}\right)$ and relative humidity $(\mathrm{RH})$ range of $\sim 0 \%$ to $100 \%( \pm 2 \%)$. The sensor was controlled via the Arduino. The sensor's positive lead voltage has a min/max of $+3.3 /+6 \mathrm{~V}$ DC so it was powered by the Arduino's $5 \mathrm{~V}$ output pin.

\section{$\underline{\text { 6. Coding and Device Timing }}$}

For the RRA to function as desired it was necessary for each electronic component to operate in a specific sequence with appropriate timing. As currently described, two separate programs were used to control the RRA: the Arduino IDE and an FCL executable. To ensure commands were executed at the appropriate time, a top-level "master code" communicated between both the FCL Controller and the Arduino. The master code was written using Python 3.8.2.

The Arduino was not directly driven by the Python code, but instead through a series of serial commands that the Python code sent to the Arduino IDE. Using this method, it was necessary to have two sets of code: functions written using the $\mathrm{C}$ programming language in the Arduino IDE and calls for these functions in the Python master code. 
To demonstrate, Figure 17 and Figure 18, respectively, show the Arduino and Python code necessary for Python to command the Arduino to switch an LED light on and off. The Python code starts by initializing a serial connection with the Arduino. Once the Arduino turns on, the serial monitor begins and waits for user input. The Arduino code contains two functions: one for turning on and another for turning off the LED. In this example, the character " $\mathrm{L}$ " is the command for turning the light on and the character " $\mathrm{D}$ " is the command for turning the light off. The Python code also contains two functions for controlling the LED: to turn the light on it sends the character "L" to the Arduino as a byte, and to turn the light off it sends the character " $D$ " as a byte. The while loop at the end of the Python code calls both functions with a delay of one second in between, resulting in the light flashing on and off. Using the described method, each component controlled by the Arduino was called using Python.

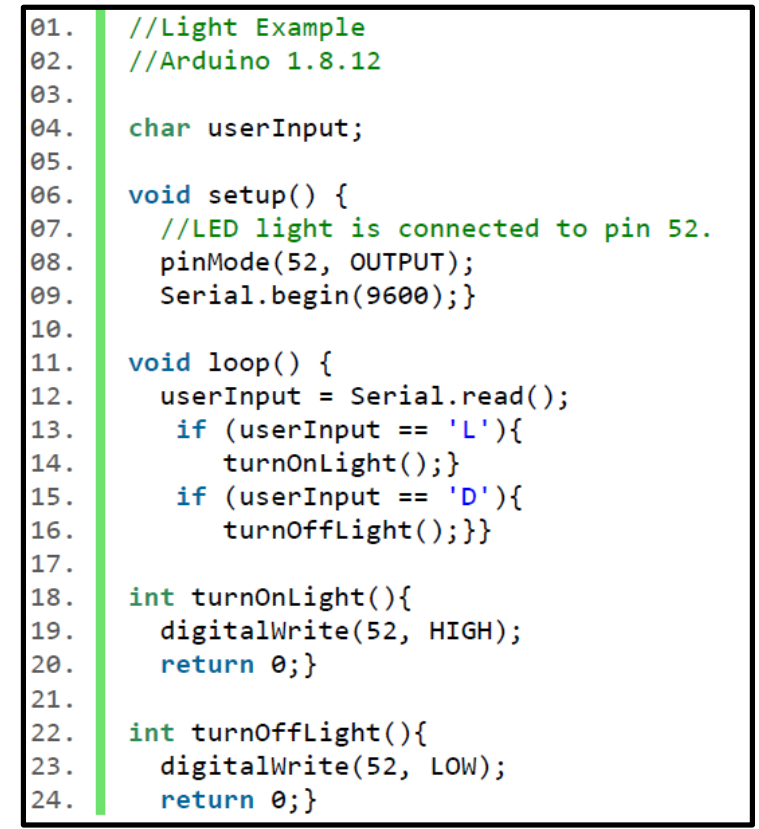

Figure 17. Example Arduino code to demonstrate a serial connection with Python. Code waited for commands from Python and then executed the called function. 


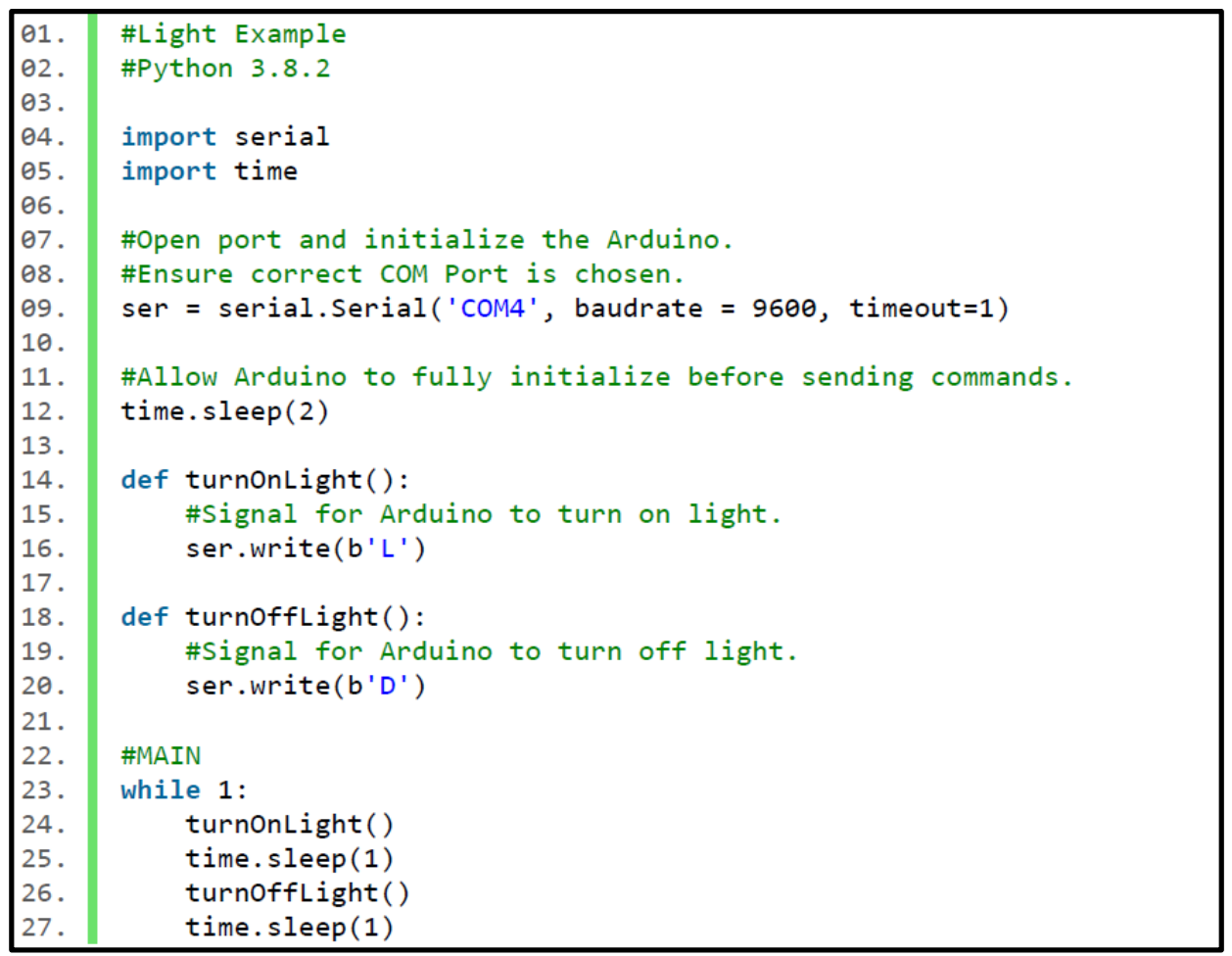

Figure 18. Example Python code to demonstrate serial connection with the Arduino.

Code sends commands to Arduino which are then executed.

A Python module called "subprocess" was used to aid in communication with the FCL Controller. The subprocess module provided the ability to spawn new processes and connect to their input/output/error pipes. Example code for running the printer using the subprocess module is found in Figure 19. The code starts by opening the FCL's Python code and allowing the Controller to initialize. The command for the FCL Controller to dispense a droplet, as coded in the FCL, is "run". This text command must be converted to UTF-8 (8-bit Unicode Transformation Format) to be sent to the FCL program. Once the code is sent, the subprocess closes and the Controller dispenses a droplet. 


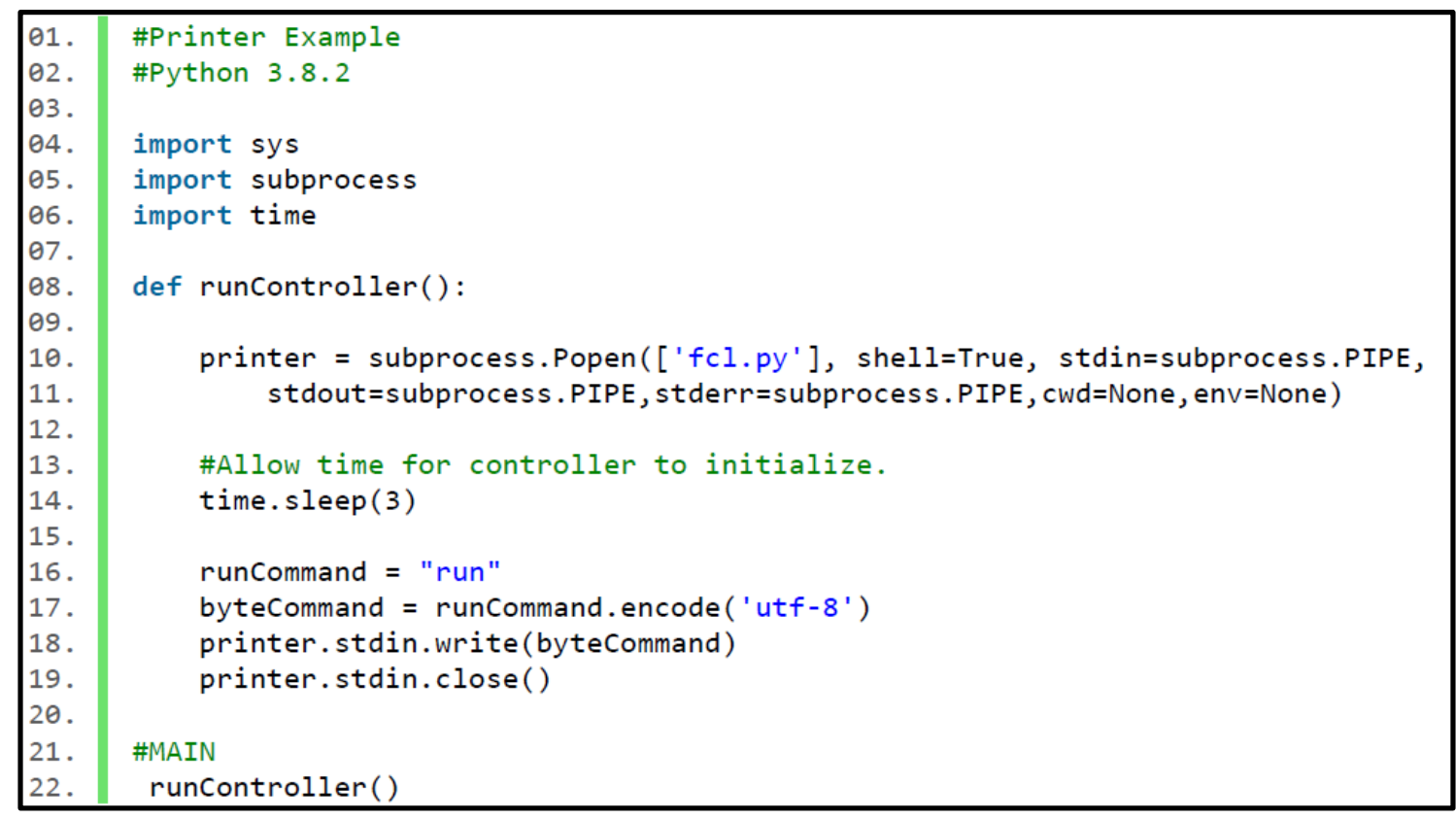

Figure 19. Python example code for using subprocess module to control FCL controller.

Using the serial library and subprocess library, it was possible to create the previously mentioned master code. The master code contained the functions necessary to control both the Arduino and FCL Controller in the proper sequence. The master code and Arduino code are found in Appendix E and Appendix F, respectively. Once the functions were written and communication between devices established, attention was brought to the timing of the code. The proper sequence of events was as follows:

1. Turn the motor $x_{\text {step }}$ number of steps to move the film forward.

2. Dispense a new droplet.

3. Turn on the LED light ring.

4. Capture image of a previously dispensed, evaporated droplet.

5. Turn off the LED light ring.

6. Record environmental conditions.

7. Pause until $t_{\text {next }}$ is reached. Repeat. 
In continuous operation, the sequence of events repeated indefinitely. However, some variations of the sequence were made to account for startup and shutdown conditions. The master code asked the user to input a desired number of droplets to be dispensed and then looped through modified sequences until all the drops were imaged. Figure 20 demonstrates the three dispensing conditions which must be accounted for by altering the code sequence.

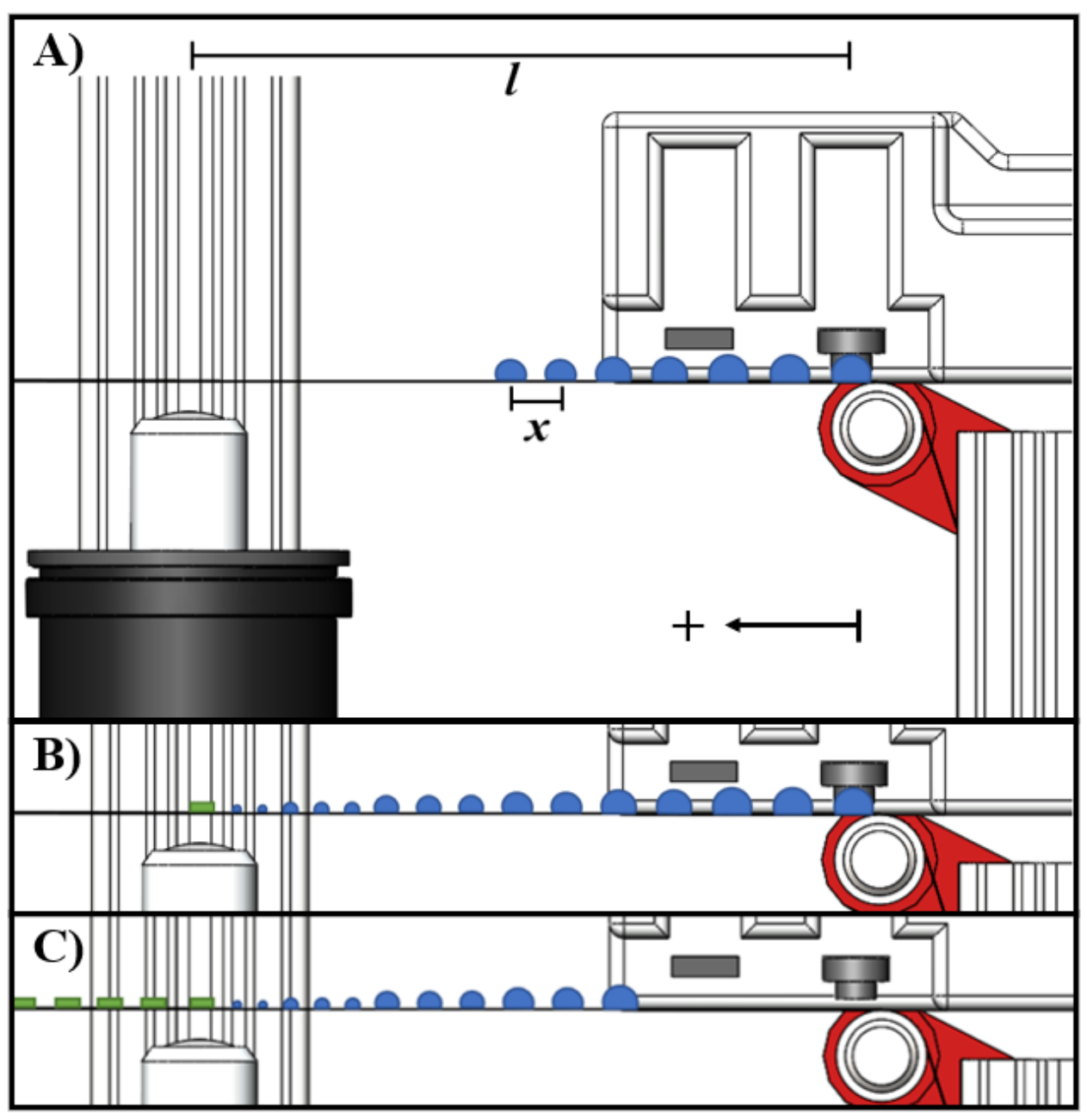

Figure 20. Three states of the dispensing process: A) Droplets have been dispensed but have not yet reached the camera for imaging. B) Droplets have reached the camera for imaging and are still dispensing. C) All droplets have been dispensed but not all imaged. 
When the droplets first began dispensing and had not yet reached the camera, the startup sequence was used to avoid capturing blank images. Once the droplets reached the camera, all seven steps of the sequence were repeated - this was the steady state sequence of the apparatus. Finally, once the desired number of droplets was dispensed, the printing function of the sequence was skipped, and the new shutdown sequence repeated until all droplets were imaged. Timing of the functions within each sequence is shown in Table 2.

Table 2. Timing of functions for startup, steady state, and shutdown sequences for a travel distance of 115.5 millimeters, 7 millimeters between droplet centers, and assumed droplet evaporation time of 15 minutes.

\begin{tabular}{|c|c|c|c|}
\hline \multirow{2}{*}{ Function } & \multicolumn{3}{|c|}{ Function Timing (s) } \\
\cline { 2 - 4 } & $\begin{array}{c}\text { Startup } \\
\text { Sequence }\end{array}$ & $\begin{array}{c}\text { Steady State } \\
\text { Sequence }\end{array}$ & $\begin{array}{c}\text { Shutdown } \\
\text { Sequence }\end{array}$ \\
\hline Turn Motor & 0.19 & 0.19 & 0.19 \\
\hline Pause & 2.00 & 2.00 & 0.00 \\
\hline Print & 9.00 & 9.00 & 0.00 \\
\hline Light On & 0.00 & 0.00 & 0.00 \\
\hline Pause & 0.00 & 2.00 & 2.00 \\
\hline Cam Shutter & 0.00 & 0.15 & 0.15 \\
\hline Pause & 0.00 & 2.00 & 2.00 \\
\hline Collect Ambient & 0.30 & 0.30 & 0.30 \\
\hline Light Off & 0.00 & 0.00 & 0.00 \\
\hline Time until Next & 44.76 & 40.61 & 51.61 \\
\hline Total & $\mathbf{5 6 . 2 5}$ & $\mathbf{5 6 . 2 5}$ & $\mathbf{5 6 . 2 5}$ \\
\hline
\end{tabular}


The total time between drops was a function of droplet evaporation time, the distance between the camera objective and printhead, and the distance between droplet centers, as depicted in Figure 20. The maximum number of droplets that fit on the film between the cartridge printhead and objective lens is found using

$$
n=\left\lfloor\frac{l}{x}\right\rfloor
$$

where $l$ is the distance between the center of the objective lens and center of the FCL Controller cartridge, and $x$ is the distance between droplet centers. The total waiting time between consecutively dispensed droplets is given by the equation

$$
t_{\text {next }}=\frac{t_{\text {evap }}}{n}
$$

where $t_{\text {evap }}$ is the total time for a droplet to evaporate.

The distance between droplet centers, $x$, must be carefully selected to ensure minimal positional error between an evaporated droplet and the objective lens. The stepper motor has a finite resolution by which the distance $l$ may not be divisible; therefore, the positional error between successive droplets accumulates and results in the droplet becoming misaligned with the objective lens. With the current code, it was not possible to correct for overall positional error by altering the number of steps between successive droplets. By carefully selecting $x$, the total positional error may be remedied by slight alterations to the RRA. Finding the optimal distance between droplet centers is as follows: 
The distance travelled per stepper motor step is calculated using

$$
m=\frac{\pi D \theta_{m}}{360 E}
$$

where $D$ is the diameter of the capstan, $\theta_{m}$ is the full step size of the stepper motor in degrees, and $E$ is the excitation mode. The distance between the center of the objective lens and the center of the cartridge printhead can be converted to steps using

$$
l_{\text {step }}=\frac{l}{m}
$$

and the number of steps between drops using

$$
x_{\text {step }}=\frac{l_{\text {step }}}{n}
$$

It should be noted that due to the floor function in Equation 1,

$$
x_{\text {step }}=\frac{l_{\text {step }}}{n} \neq \frac{x}{m}
$$

Since the stepper motor can only step in integer values, $x_{\text {step }}$ must be rounded so that

$$
r_{\text {step }}=\left\lceil\frac{\left\lfloor 2 x_{\text {step }}\right\rfloor}{2}\right\rceil
$$

Finally, the total positional error of the motor can be calculated using

$$
\varepsilon=\left(r_{\text {step }}-x_{\text {step }}\right) * n m
$$


If a droplet's final position lies beyond the the objective lens, Equation 7 yields a positive number. If a droplet's final position is short of the objective lens, Equation 7 yields a negative number. Using Equation 7, plots of the motor's theoretical positional error for each excitation mode were generated and are found in Figure 21.

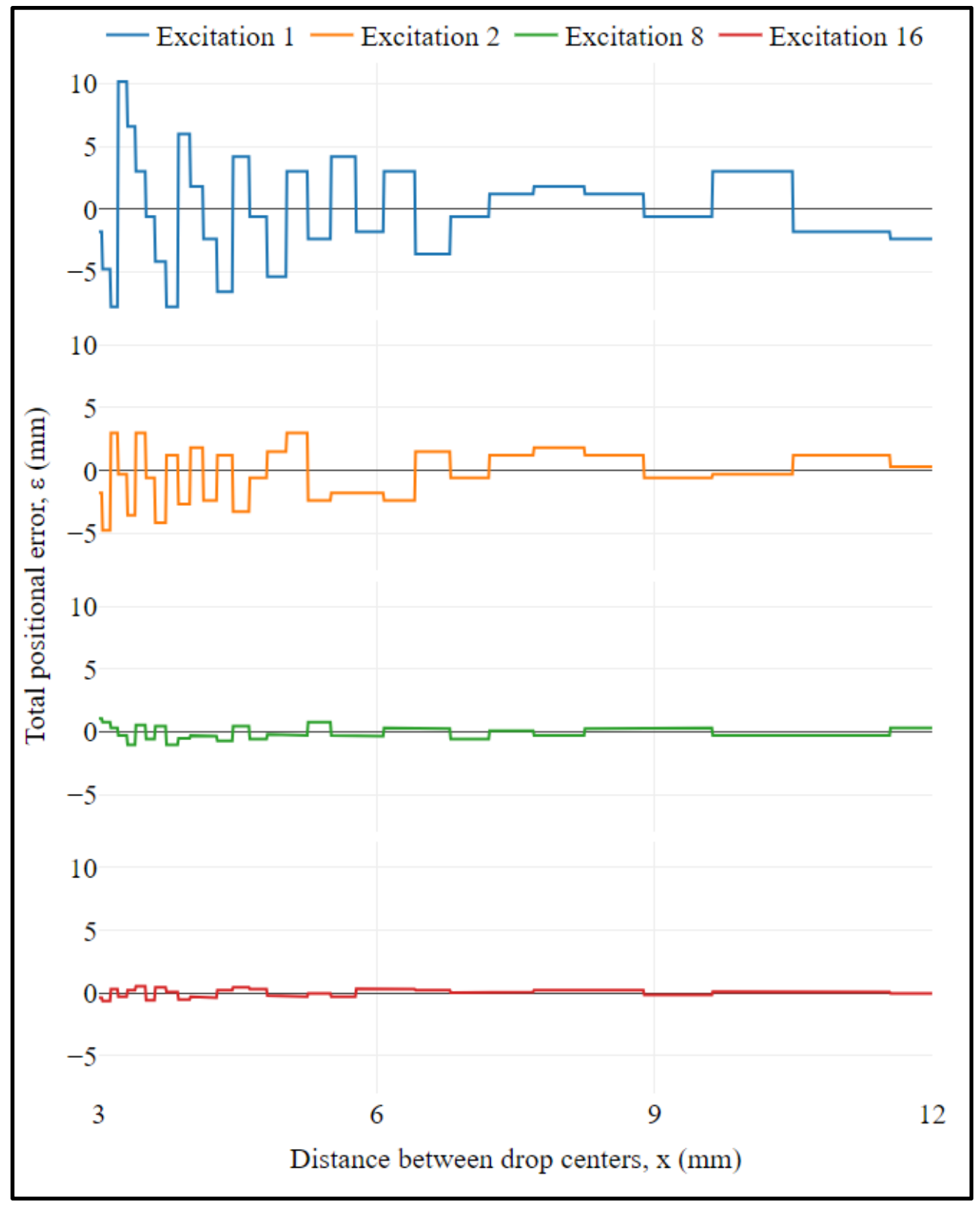

Figure 21. Motor's total positional error for an $l$ of 115.5 millimeters, $D$ of 38.1 millimeters and $\theta_{m}$ of 1.8 degrees. 
As seen in Figure 21, the total positional error of the droplet in relation to the objective lens decreased with increasing excitation. Using these plots, it was possible to select an $x$ value to minimize the positional error. In this case, using an excitation of 16, an $x$ between 6.80- and 7.21-millimeters yielded a positional error of only 0.005 millimeters.

Another consideration for picking $x$ is the maximum number of droplets between the objective lens and printhead. Using the previously mentioned $x$ values, $n$ was 16 drops. Depending on time constraints, it may be beneficial to have a greater number of droplets if positional error is negligible (i.e., the droplets remain in the camera's field of view). As shown in Figure 22, the time to dispense a given number of droplets is inversely proportional to the maximum number of drops on the film, $n$. For this reason, instead of picking a $x$ with the smallest $\varepsilon$, it may be beneficial to choose a smaller $x$ and then correct the error by modifying the test apparatus. It is recommended to use an $x$ greater than 4.0 millimeters to prevent overlap of droplets, which are, depending on wettability of the surface, approximately 1.5 millimeters in diameter for 1.0 microliter droplets and 2.0 millimeters in diameter for 2.0 microliter droplets. 


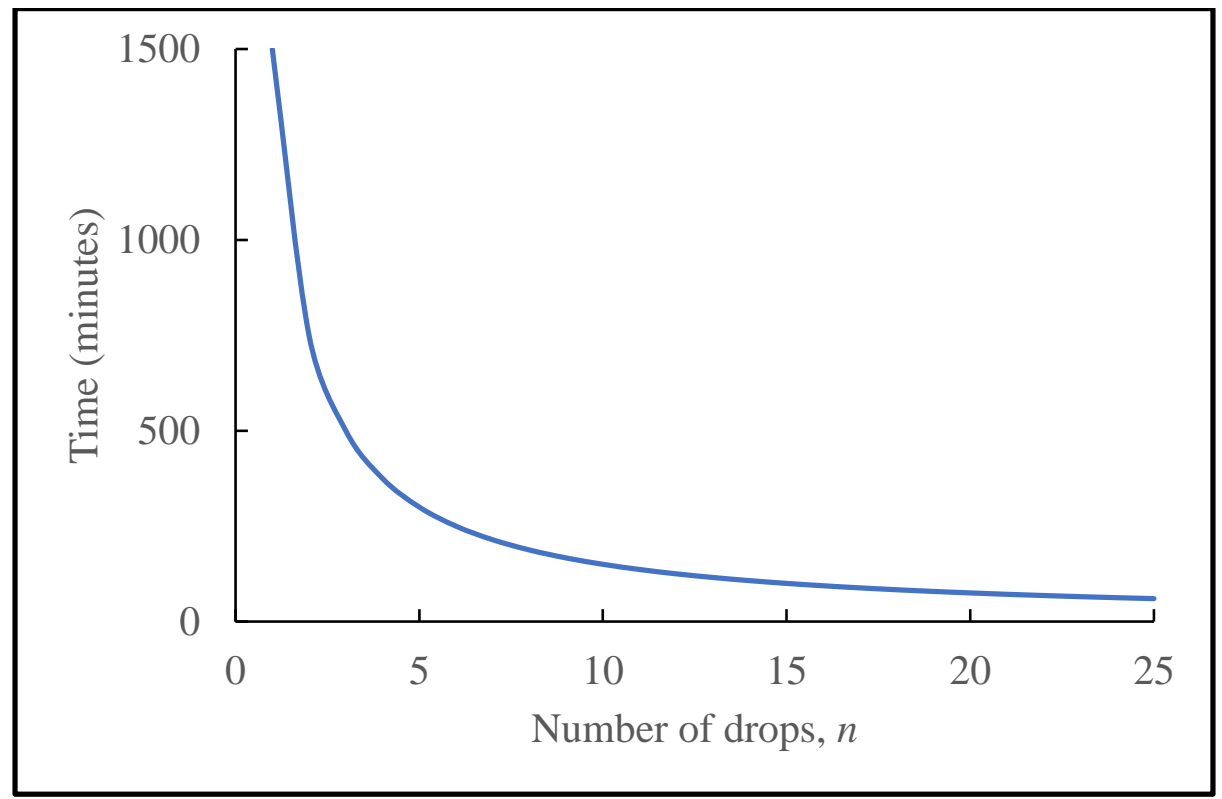

Figure 22. Time to dispense and collect 100 droplets during continuous operation.

Assuming a droplet evaporation time of 15 minutes.

Once a proper $x$ value was selected, Equation 6 was used to determine the number of steps to be taken between successive droplets. The correct number of steps must be changed in the Arduino code before beginning the experiment. Equation 2 must also be used to determine the time between successive droplets, $t_{\text {next }}$, and the "Time until Next" must be updated for each sequence in the Python master code. 


\section{Whiskey Sample Analysis}

Testing was conducted using commercially purchased Buffalo Trace Kentucky Straight Bourbon Whiskey (BT), 45\% alcohol by volume (ABV). As shown in previous work (Williams et al., 2019), whiskey webs only formed in a certain range of ABV, so the sample was diluted to $25 \% \mathrm{ABV}$ using deionized (DI) water. The volume of DI water necessary to dilute a whiskey to a desired ABV is found using

$$
V_{\text {water }}=\frac{A B V_{d}}{A B V_{o}} * V_{f}
$$

where $\mathrm{ABV}_{\mathrm{d}}$ is the desired $\mathrm{ABV}, \mathrm{ABV}_{\mathrm{o}}$ is the original $\mathrm{ABV}$, and $\mathrm{V}_{\mathrm{f}}$ is the final volume of solution. The amount of whiskey sample needed can then be found using

$$
\mathrm{V}_{\text {whiskey }}=\mathrm{V}_{\mathrm{f}}-\mathrm{V}_{\text {water }}
$$

Performance of the Controller is determined not only by the code parameters and chipset (nozzles/heaters), but also by the physical properties of the liquid being printed. The most important properties to ensure compatibility with the Controller cartridge are surface tension, viscosity, and $\mathrm{pH}$ of the test sample. The static surface tension of the sample was measured using a Kibron AquaPi portable micro-tensiometer, the viscosity was measured using a Nametre Viscoliner, and the $\mathrm{pH}$ was tested using a HANNA edge $\mathrm{pH}+$. Values for each are shown in Table 3. Interestingly, the diluted sample of Buffalo Trace had a lower $\mathrm{pH}$ than the undiluted sample, which was likely due to impurities in the DI water. 
Table 3. Surface tension, viscosity, and pH for Buffalo Trace Kentucky Straight Bourbon Whiskey.

\begin{tabular}{|c|c|c|}
\cline { 2 - 3 } \multicolumn{1}{c|}{} & Undiluted (45\% ABV) & Diluted (25\% ABV) \\
\hline $\begin{array}{c}\text { Surface tension } \\
\text { (millinewtons/meter) }\end{array}$ & 31.02 & 38.51 \\
\hline Viscosity (centipoise) & 2.77 & 2.19 \\
\hline pH & 4.09 & 3.90 \\
\hline
\end{tabular}

\section{E. Device Testing}

\section{Cartridge Selection}

To test the viability of thermally jetting whiskey webs using the FCL controller, a short and tall cartridge were both filled with the sample and printed onto the FEP film. The short cartridge held approximately 60 microliters without overfilling. The tall cartridge held 750 microliters without overfilling. Each cartridge was placed into a port on the FCL Controller and dispensed five 1.0 microliter droplets using the default Controller settings found in Table 1 . Ambient conditions were $21^{\circ} \mathrm{C}$ and $21 \%$ relative humidity. After the droplets evaporated, they were examined using an inverted microscope (Nikon Ti-U). The droplets dispensed by the short cartridge formed webs for $80 \%$ of the droplets while the tall cartridge did not produce webs.

Previous studies demonstrated filtration of American whiskeys has an impact on the web coverage of evaporated droplets (Boone, 2019). It was suspected that either the felt filter or the stainless-steel mesh were preventing the constituents necessary to form a 
whiskey web from being dispensed. The felt filter was removed from two of the tall cartridges and placed in a vial with five milliliters of diluted sample. After soaking for 24 hours, the felt filters were removed from the vial and the liquid contents of each were wrung into a separate vial. Five 1.0 microliter droplets of the separated liquid were dispensed onto the FEP film using a pipette, with similar ambient conditions. None of the five droplets from the sample soaked in felt produced whiskey webs.

To test if the stainless-steel mesh also prevented webs from forming, a tall cartridge with the felt removed was filled with 60 microliters of the diluted sample. The tall cartridge could not be filled to the top without the felt. The felt created a backpressure which prevented the nozzles from drooling; with the felt removed, the head pressure of the full cartridge made the nozzles drip. Five 1.0 microliter droplets were printed at similar ambient conditions and none of the droplets formed webs. It was unclear whether the filters were actively filtering out the necessary particles or if chemical reactions between the whiskey and the filters were responsible for the lack of web formations.

While the felt could be easily removed from the tall cartridge, the stainless-steel mesh could not be removed without destroying the cartridge. Since the stainless-steel mesh could not be removed, only short cartridges were used in full-system testing. Unfortunately, only two of the short cartridges were available, so they were cleaned and reused several times during preliminary trials and final tests. 


\section{Image Focus and Digital Analysis}

The LED ring light was turned on and positioned approximately two inches above the FEP film. The camera was connected to a computer via USB and Canon's EOS Utility software was used for a live viewing, remote shooting mode. A pipette was used to deposit a 1.0 microliter droplet onto the FEP film above the camera objective.

Originally the design did not account for the mechanical tube length of the objective lens. As shown in Figure 23, a Nikon 4x objective was mounted directly to the camera body using an appropriate adapter. Even when increasing the magnification with a Nikon 10x objective, the droplets appeared unexpectedly small compared to the inverted microscope used in previous studies. Droplets imaged using a $4 \mathrm{x}$ and 10x objective mounted directly to the camera body are shown in Figure 24 .

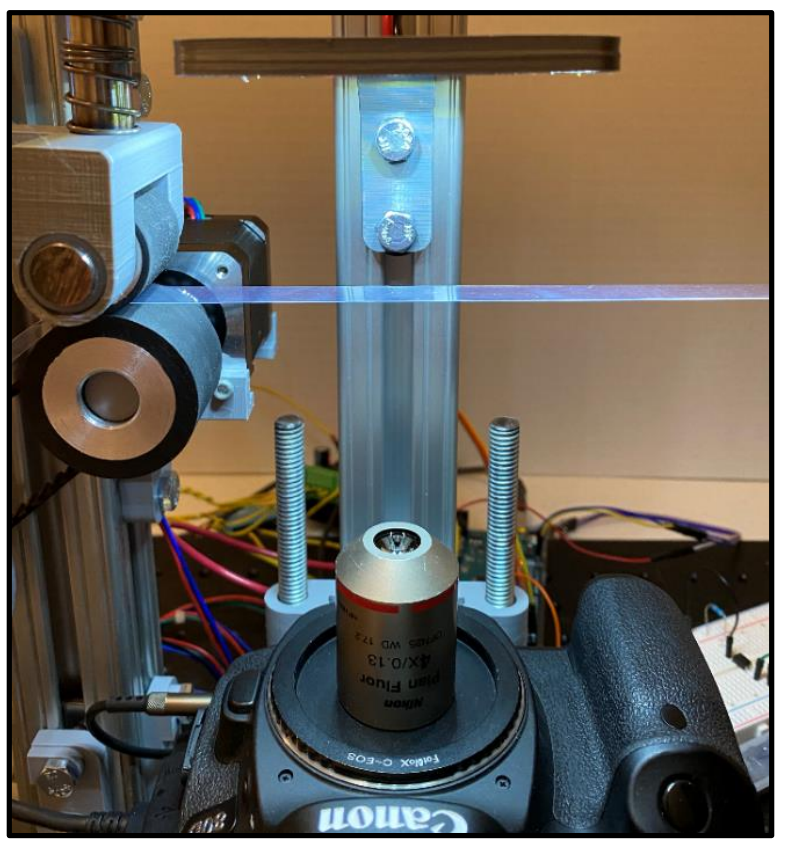

Figure 23. Nikon objective mounted directly to Canon EOS 80D camera body. 


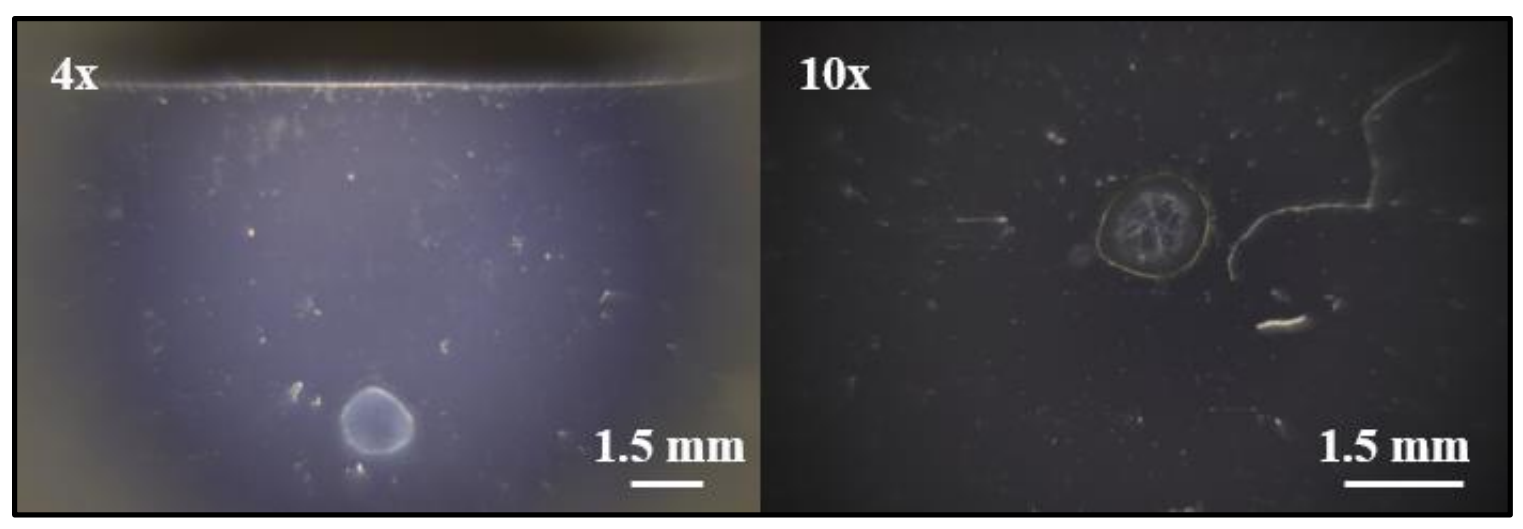

Figure 24. Uncropped images of droplets using a 4x objective lens (left) and 10x objective lens (right) mounted directly to camera body.

The length of an object in an image captured using an infinity corrected objective lens can be estimated using

$$
l_{\text {digital }}=\frac{p_{\text {sensor }}}{L_{m a g}} * l_{p x}
$$

where $p_{\text {sensor }}$ is the size of the physical pixels on the camera's image sensor, $L_{\text {mag }}$ is the magnification of the objective, and $l_{p x}$ is the length of the object measured in pixels. A Canon EOS 80D has an image sensor pixel size of 3.7 micrometers. GIMP, an open source raster graphics editor, was used to measure the number of pixels across the diameter of the 4x-magnified droplet shown in Figure 24. The diameter of the droplet was measured as approximately 615 pixels. Using Equation 10, the diameter of the droplet was estimated to be approximately 0.57 millimeters in diameter. The true diameter was measured using digital calipers (Mitutoyo Absolute Solar Digimatic) to be approximately 1.54 millimeters. The discrepancy in the estimated and measured diameters was attributed to overlooking the geometrical optics of the imaging system. As previously described, infinity-corrected 
microscopes have a predefined reference focal length which must be accounted for by an optical tube. Since the objective was mounted directly to the camera body, the image projected onto the image sensor was smaller than anticipated.

To improve image resolution and provide a means by which to measure droplet size based on digital measurements, a telescopic lens was mounted between the objective and camera body, as shown in Figure 25. As previously mentioned, the telescopic lens provided the mechanical tube length necessary for the image to be correctly projected onto the image sensor. The Nikon lenses were too large to be mounted atop the telescopic lens with the available adapters, so an American Optical 4x infinity-corrected objective lens was used instead. There were no markings on the objective indicating the mechanical tube length, so a tube length of 160 millimeters was assumed. The telescopic lens was set to approximately 160 millimeters and a new droplet was imaged. A reiterative process of increasing the focal length and digitally measuring the droplet diameter was used until calibrated to within approximately one percent of the actual droplet diameter, as depicted in Table 4. An image of the corrected 4x magnification is also shown in Figure 25 demonstrating a significant increase in imaged droplet size. 
Table 4. Estimated droplet diameter vs. measured droplet diameter using objective mounted to telescopic lens. Focal length of lens was finely adjusted until droplet size was calibrated.

\begin{tabular}{|c|c|c|c|c|}
\hline $\begin{array}{c}\text { Focal } \\
\text { Length }\end{array}$ & Diameter (px) & $\begin{array}{c}\text { Estimated } \\
\text { Diameter (mm) }\end{array}$ & $\begin{array}{c}\text { Actual } \\
\text { Diameter (mm) }\end{array}$ & $\begin{array}{c}\text { Percent } \\
\text { Difference }\end{array}$ \\
\hline 154 & 1512.7 & 1.40 & 1.57 & $-10.88 \%$ \\
\hline 163 & 1573.9 & 1.46 & 1.57 & $-7.27 \%$ \\
\hline 171 & 1625.3 & 1.50 & 1.57 & $-4.24 \%$ \\
\hline 179 & 1656.3 & 1.53 & 1.57 & $-2.42 \%$ \\
\hline 187 & 1715.1 & 1.59 & 1.57 & $1.05 \%$ \\
\hline
\end{tabular}

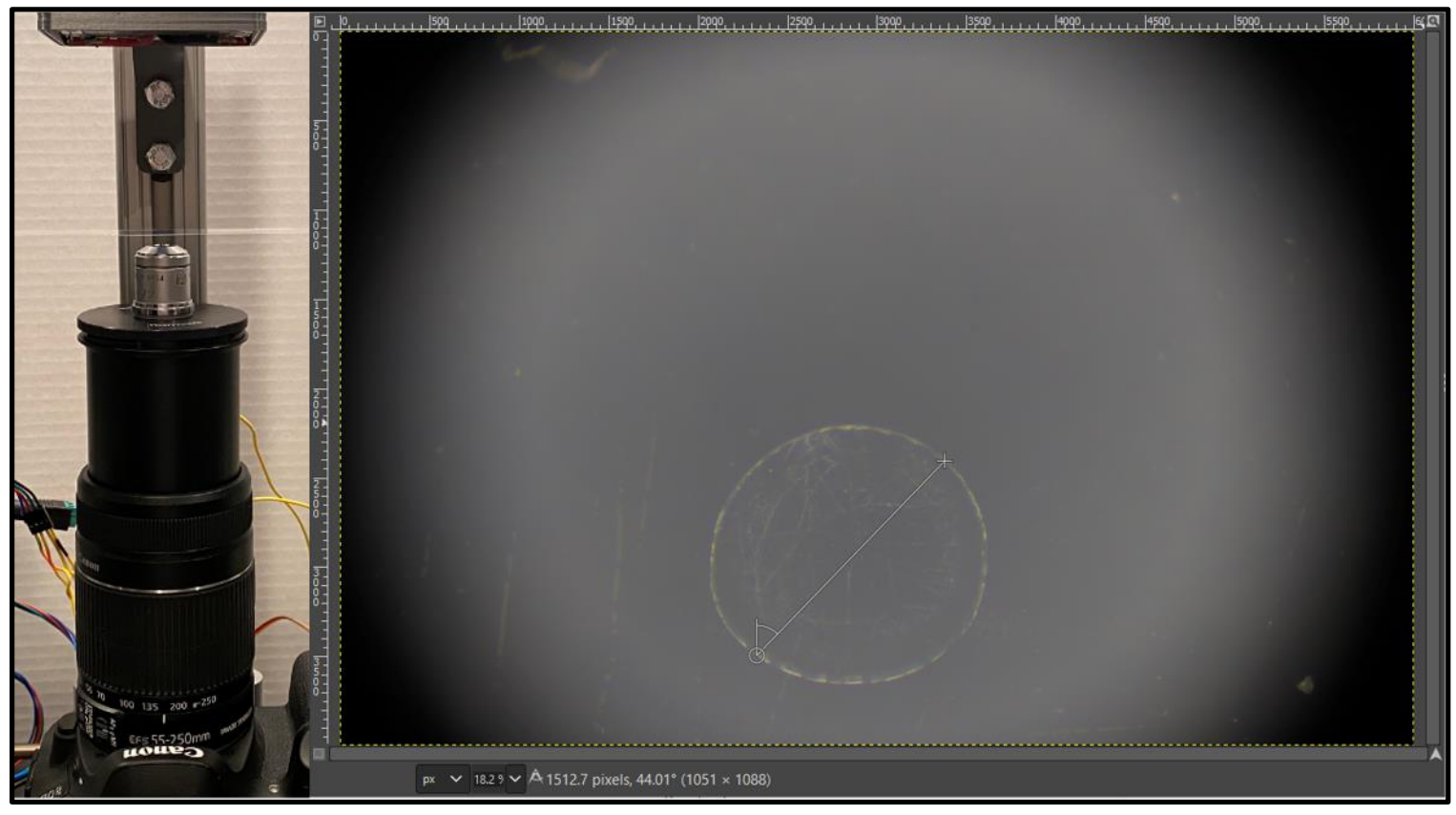

Figure 25. Uncropped image of droplet using 4x objective lens mounted to a telescopic lens to account for mechanical tube length of objective. 


\section{Droplet Illumination}

In photography, there are three complementary settings used to optimize the clarity and focus of a photo: ISO, aperture, and shutter speed. ISO is a camera's sensitivity to light. The aperture is a diaphragm inside the lens that can adjust the amount of light that is captured. The shutter speed is how long a camera allows light to hit the image sensor.

A high ISO results in brighter yet grainier images. To minimize the graininess of the droplet images, the ISO of the camera was held constant at its lowest setting of 100 . To maximize the detail captured in an image, the aperture should be at its lowest setting. For this lens, a f-stop of 5.6 was the lowest. Holding the ISO and aperture constant, the shutter speed was the only variable to optimize. For constant lighting conditions, a slow shutter speed yields a brighter image while a fast shutter speed yields a darker image.

To visualize whiskey webs, it is necessary to use scattered light. The height at which the LED ring light is above the FEP film affected the angle at which the light hit the evaporated droplet. The effectiveness of the ring light was tested at four different heights, as shown in Figure 26. The shutter speed was adjusted so that each image provided similar brightness/contrast. It was qualitatively determined that the optimal positioning of the light ring was 4 inches above the film. At 4 inches there was no reflective glare, and finer web

details were perceivable. For optimum brightness and contrast, a shutter speed of $1 / 20^{\text {th }}$ of a second was chosen. 


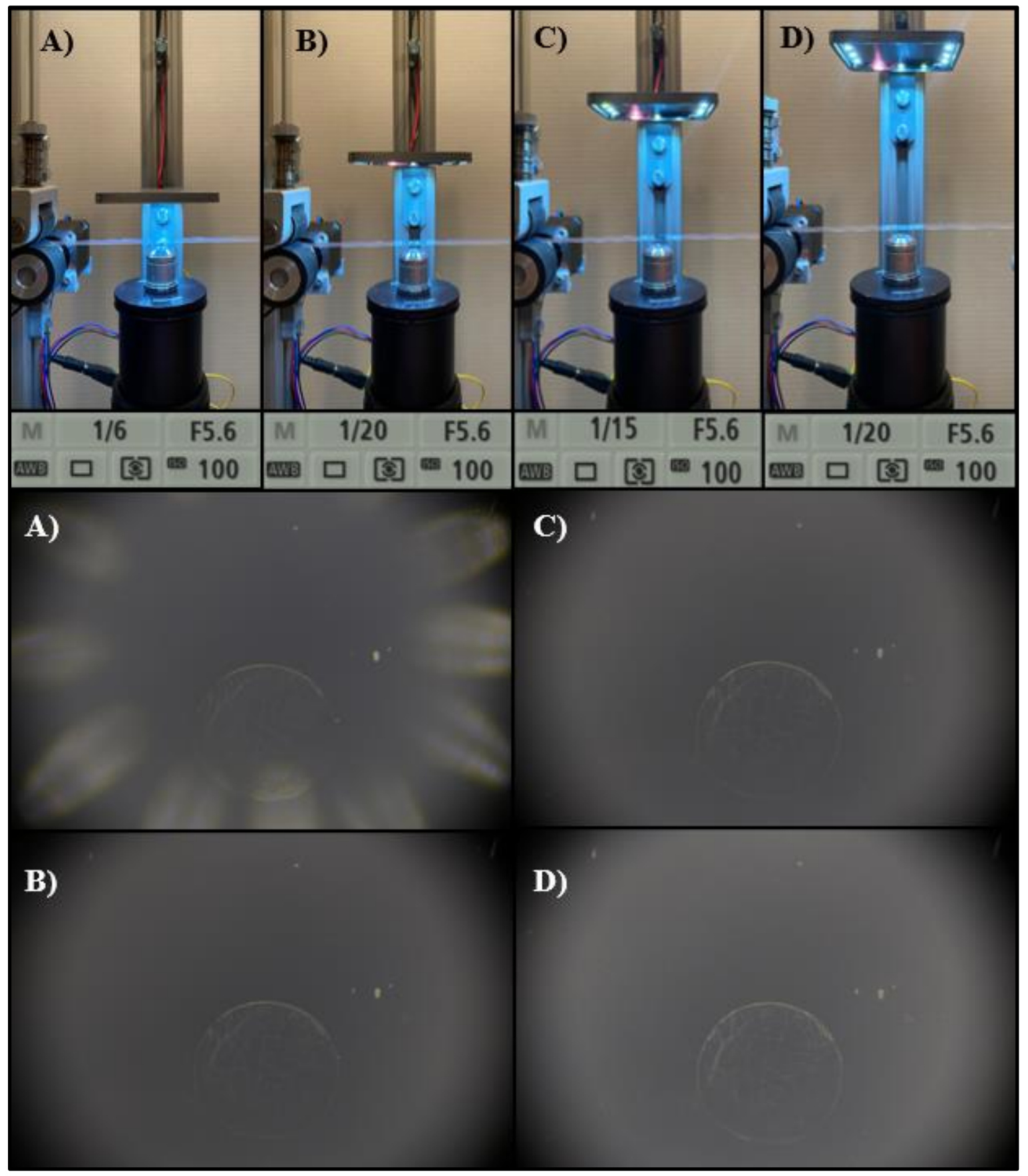

Figure 26. Images produced by adjusting camera shutter speed and position of LED ring light above the film at A) 1-inch, B) 2-inches, C) 3-inches, and D) 4-inches. 


\section{Temperature and Humidity Sensor Validation}

A ThermoPro indoor humidity and temperature monitor (Model No. TP-55) was chosen as the device to validate the DHT22 sensor readings. The ThermoPro claimed a thermal accuracy of $\pm 1.0{ }^{\circ} \mathrm{C}$ and a relative humidity measurement accurate to $\pm 3 \%$.

\section{Stepper Motor Accuracy Validation}

Repeatable and consistent alignment of evaporated droplets with the objective lens was crucial for successful data collection. Since the motor was directly responsible for the alignment of the droplets, the accuracy of its rotation was tested. Figure 27 shows the setup for the positional accuracy testing of the stepper motor. A datum was 3D printed and mounted to the center column of the RRA. Blue masking tape was aligned with the edge of the datum and adhered to the film. Using an excitation mode of 16, the stepper motor was programmed to take 193 steps, equivalent to 7.0 millimeters. The distance between the

datum and the masking tape was measured using digital calipers and repeated for a theoretical total of 70 millimeters of travel. 


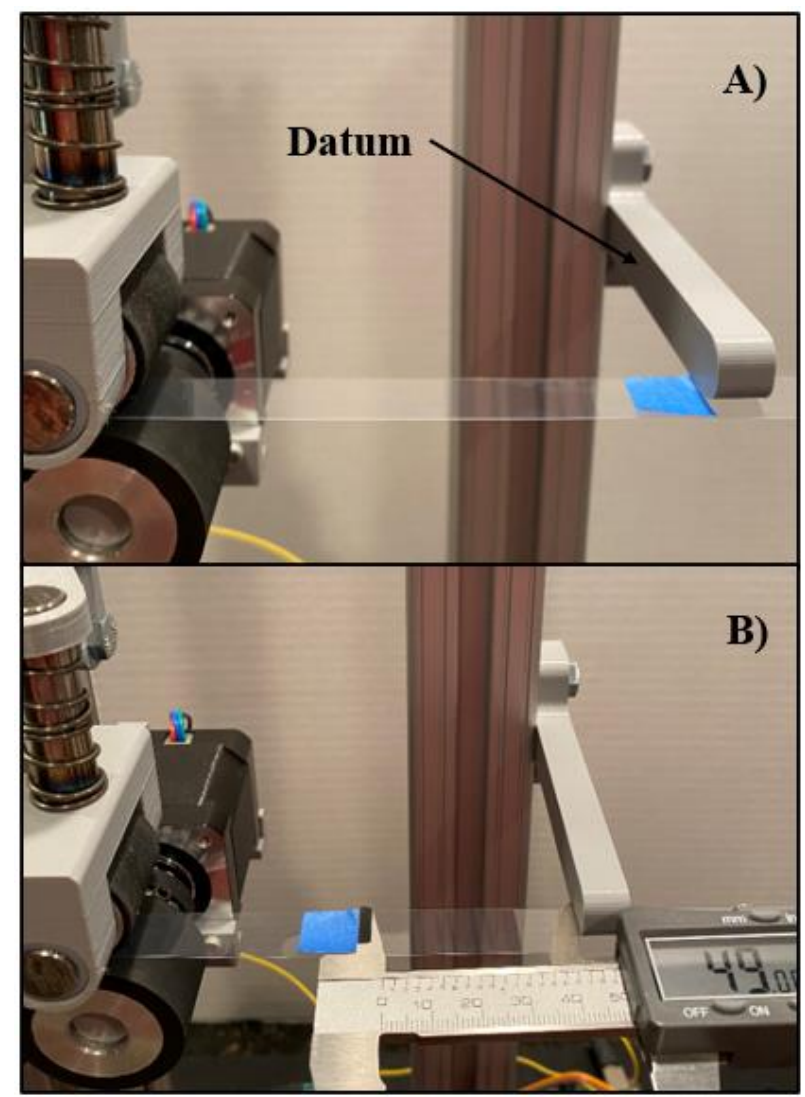

Figure 27. Stepper motor positional accuracy test setup. A) Start of test, tape aligned with datum. B) Several runs into test, tape is pulled forward by motor and the distance from the datum measured with digital calipers.

\section{$\underline{\text { 6. Film Tension Test }}$}

To ensure the maximum holding torque of the motor was not exceeded, a test was devised to measure the tension in the film so that the static torque applied to the motor could be calculated. As shown in Figure 28, a weight was suspended at the center of the film between the capstan and the supply reel holder. 


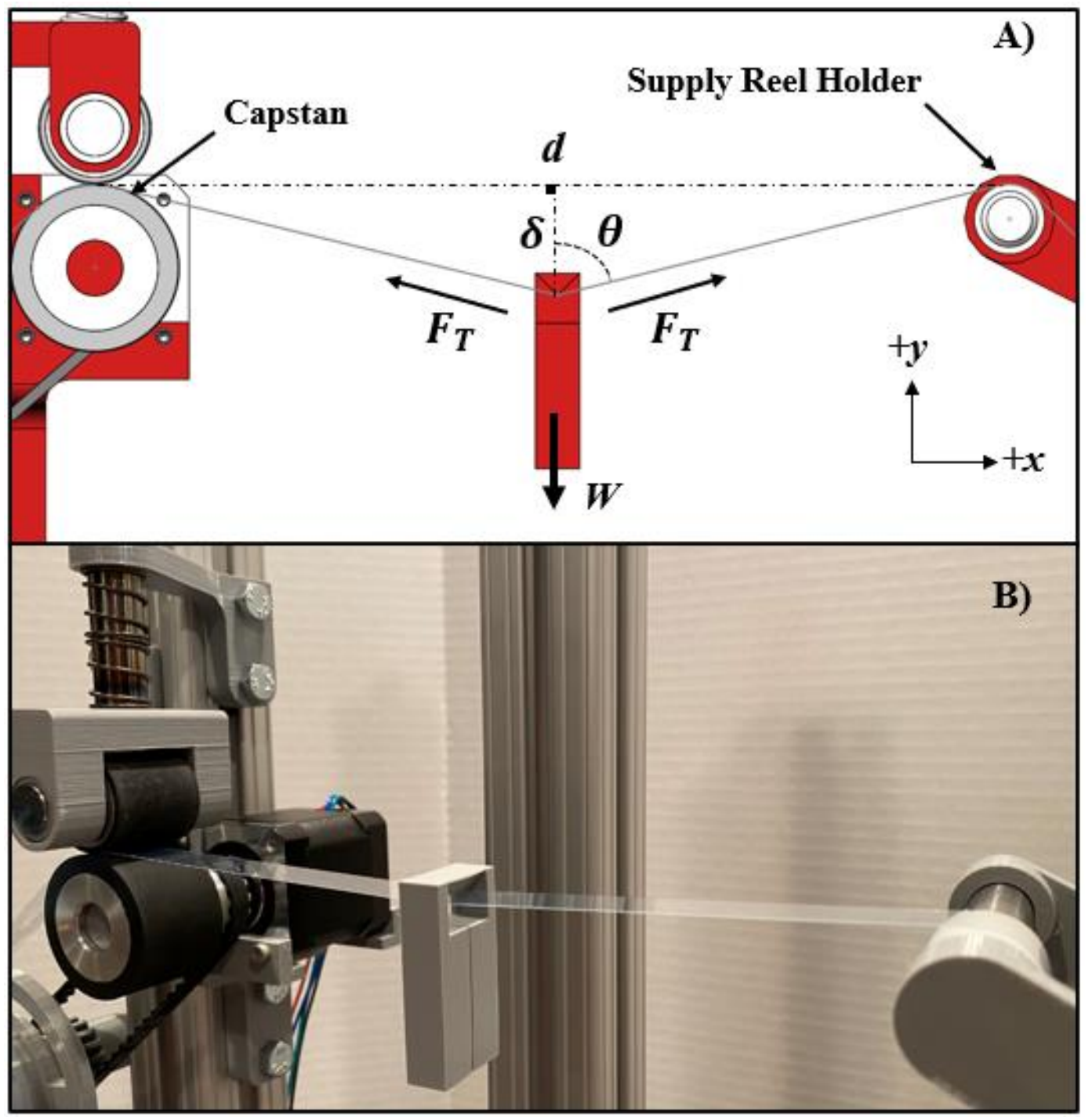

Figure 28. A) Free body diagram of tension being measured by suspending a known weight from center of film. B) Weight being suspended from film in actual testing.

The deflection of the film caused by the weight, $W$, was measured and the tension in the film was calculated using the force balance equation

$$
2 F_{T} \cos \theta=W
$$


where $\theta$ is the angle between the $y$-axis and the film. Using trigonometric identities, Equation 11 was simplified to

$$
F_{T}=\frac{W}{2 \delta} \sqrt{\delta^{2}+\frac{d^{2}}{4}}
$$

where $\delta$ is the deflection of the film and $d$ is the distance between the capstan and the supply reel holder.

Polylactic acid (PLA), the filament used to 3D print the custom parts for the RRA, has a density of 1.94 grams/cubic centimeter (Granta Design Limited). The weight shown in Figure 28 was designed to have a volume of 8.07 cubic centimeters, equating to approximately 10 grams. The weight had a slot through the base to allow for it to be hung without removing the film from the assembly. The part was printed using $100 \%$ infill and weighed using a digital scale (Sartorius BP211D). The actual weight was 9.61832 grams, or approximately 0.34 ounces. Assuming no slippage, the torque applied on the motor can be calculated using

$$
T=F_{T} r
$$

where $r$ is the radius of the capstan. 


\section{Printhead Failure Analysis}

Images of evaporated droplets were assessed and deemed successful if a web pattern formed. Droplets that did not form a web pattern were deemed failures. The success rate of a testing method was evaluated as the percentage of droplets that formed a web pattern. In assessing the cause of web formation failure or droplet abnormalities, it was necessary to analyze the printhead for modes of failure.

Printheads experience an increasing failure rate - the longer they are used, the more likely they are to fail. There are two common printhead failure modes which can be examined using a microscope: kogation and throat blockage. Kogation is the gradual degradation of printhead heaters due to impurities in the jetting fluids. The impurities in the jetting fluid bake onto the heating elements of the printhead, causing surface roughness and reducing the heat transfer into the jetting fluid. If kogation is serious enough the buildup of impurities on the heater can lead to unstable bubble formation, or prevent nucleation altogether (Shirota, Shioya, Suga, \& Eida, 1993). Throat blockage occurs from contaminants blocking the flow of fluid from the reservoir to the individual nozzles.

After testing, the cartridge was removed from the FCL controller and without being cleaned, examined under a microscope (Nikon MM-60) using various magnifications. The printhead was imaged and then cleaned using a solution of $95 \%$ ethanol. The reservoir was filled completely with the ethanol and the bottom of the chip was dabbed using a cotton swab until the ethanol had completely evaporated from the reservoir. The printhead was then reexamined to determine if the kogation or blockages had been removed, and imaged. 


\section{$\underline{\text { F. Droplet Testing }}$}

Droplet testing can be broken into three main testing procedures: Manual Drop Testing, Continuous Drop Testing, and Single Drop Testing. Throughout testing, the FCL Controller parameters were altered to be a variation of the parameters given in Table 1 . The specific parameters for each test will be given in the results and discussion. All tests were conducted using the BT sample diluted to $25 \% \mathrm{ABV}$.

New cartridges do not require cleaning before use. Cartridges being reused should be cleaned with the method described in Printhead Failure Analysis. When a cartridge is first filled, it must be primed to ensure air bubbles have not prevented fluid from filling each nozzle. The cartridge can be primed by gently dabbing the printhead with a lint free towelette such as a Kimtech wipe. Once primed, a cap should be placed on the cartridge to help prevent evaporation, as shown in Figure 29, and then inserted into the FCL Controller.

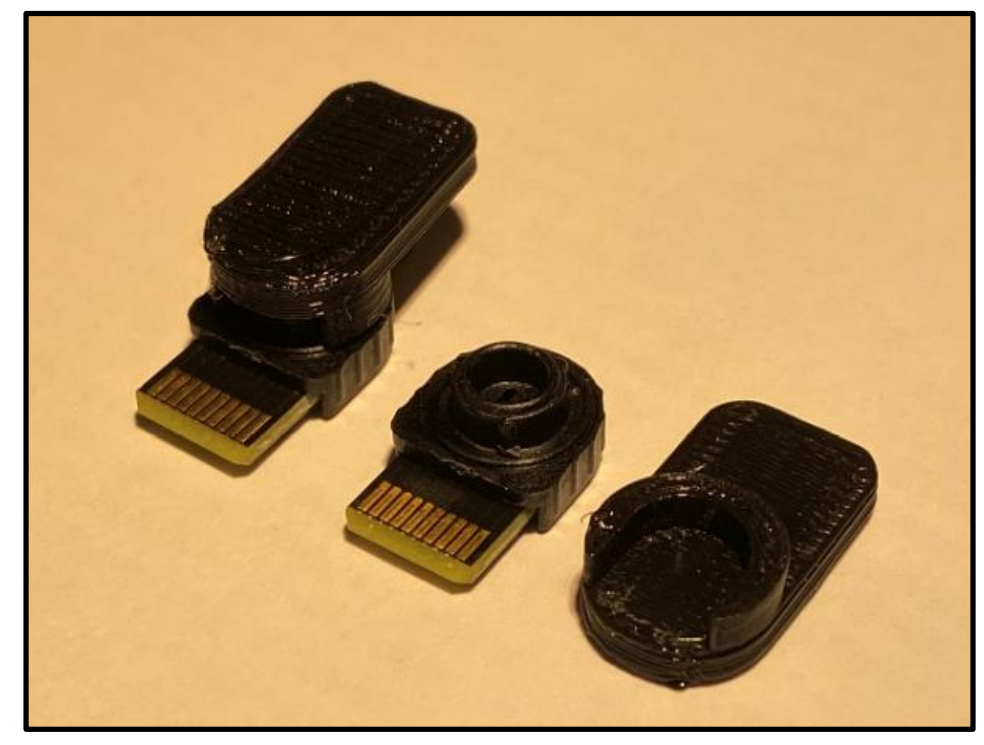

Figure 29. Caps 3D printed to reduce evaporation from cartridge reservoir. 


\section{Manual Droplet Testing}

Manual Droplet Testing (MDT) was used as the control group in this study. MDT mimics the method used in previous studies of using a pipette to manually deposit 1.0 microliter droplets onto a substrate (Carrithers et al., 2020). The substrate used in this study

was the FEP film. The FCL Controller was removed from the RRA and twenty 1.0 microliter droplets were deposited onto the FEP film using a manual pipette (Eppendorf Research plus 0.1-2.5 microliters). Temperature and humidity conditions were recorded using a ThermoPro monitor at the dispense of each droplet. Once the droplets evaporated, they were individually aligned with the camera objective by manually turning the capstan. The LED ring light was turned on and the EOS Utility Live View shooting mode was used to manually record the images. Evaporation time of each droplet was recorded.

\section{Continuous Droplet Testing}

Continuous Droplet Testing (CDT) functions as the device was conceived, with droplets being dispensed and moved along before completely evaporated, akin to continuous flow manufacturing. The motor was driven using an excitation mode of 16 with a distance between droplet centers, $x$, of 7 millimeters. Seven millimeters of travel equates to 193 sixteenth steps and results in a total positional error of 0.005 millimeters. The maximum number of droplets on the film, $n$, is 16 . Droplet evaporation time was assumed to be 15 minutes, so the master code was edited to have a $t_{\text {next }}=56.25$ seconds. 
One short cartridge was cleaned and then filled with 60 microliters of the diluted BT sample. Once capped, the cartridge was inserted into the rightmost port of the FCL Controller. Twenty 1.0 microliter droplets were requested to be dispensed by the Controller using the master code. Every ten droplets the cap was removed, and the cartridge was refilled with approximately 20 microliters of the sample. Temperature and relative humidity were recorded using the DHT sensor, and images were captured automatically. Evaporation times were not recorded due to difficulty in monitoring multiple droplets simultaneously.

\section{Single Droplet Testing}

It was hypothesized that movement of the stepper motor may cause the FEP film to vibrate and disrupt the monolayer collapse mechanisms that result in whiskey web patterns. Single Drop Testing (SDT) was devised to isolate film vibration as a potential source of error in web pattern repeatability. In SDT the motor did not move until the dispensed droplet was fully evaporated, so that any vibrations in the tape transport system did not potentially affect the collapsing mechanisms of the droplet's monolayer.

Allowing a droplet to fully evaporate before continuing greatly increased the time of the test - being equivalent to an $n$ equal to 1 in reference to Figure 22. To decrease the testing time, two ports of the FCL Controller were utilized. The reel-to-reel system was originally designed to use only one port on the FCL Controller. As shown in Figure 30, the ports of the FCL Controller were spaced at their centers by a distance of 25.4 millimeters,

which was not divisible by the resolution of any of the motor's excitation modes. This 
indivisibility meant the droplets would become offset, making it impractical to use for continuous dropping. However, manually turning the capstan allowed for droplets to be aligned with the camera objective.

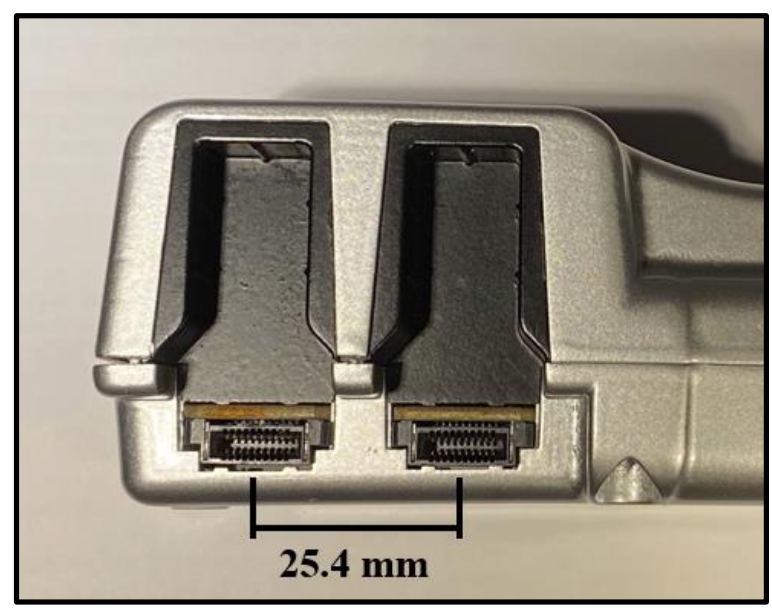

Figure 30. Spacing of ports on FCL Controller.

Two short cartridges were cleaned and then filled with 60 microliters of the diluted BT sample. Once capped, each cartridge was inserted into a port of the FCL Controller. Every 10 droplets the caps were removed, and 20 microliters of the sample were added to refill the well. The FCL Controller was controlled manually using the FCL program provided by FLTC. Temperature and humidity conditions were recorded using the ThermoPro monitor at the dispense of each droplet. Upon the evaporation of each droplet, the capstan was turned by hand so that each droplet advanced by approximately 5 millimeters. Once the droplets from the rightmost port reached the leftmost port, the film was advanced until empty film was available for deposition. The droplets were manually aligned with the camera, the LED ring light turned on, and the Canon EOS Utility Live View shooting mode was used to manually record the images. A total of 32 droplets (16 from each cartridge) and their evaporation times were recorded. 


\section{Single Droplet Testing: Single Nozzle Variant}

The Single Nozzle Variant (SNV) of Single Droplet Testing was devised to analyze the dispensing properties of individual nozzles. Just as the standard SDT, the Single Nozzle Variant waited for full droplet evaporation before movement of the FEP film to eliminate film vibrations as a variable for error. SNV differentiates from the SDT in that it only used a single nozzle for deposition of an entire 1.0 microliter droplet instead of all 16 nozzles in the primitive. A single Primitive of the FCL Controller was chosen and the incremental address was set to 16 so that only one nozzle was used. The address started at 1 and was incremented by 1 for each droplet until all 16 addresses were used. Evaporation time was recorded, and temperature/humidity measurements were recorded from the ThermoPro monitor at the dispense of each droplet. The Canon EOS Utility Live View shooting mode was used to manually record the images. 


\section{CHAPTER VI. RESULTS AND DISCUSSION}

Because the printheads experience an increasing failure rate and the same two cartridges were used throughout testing, the results gathered are not an accurate representation of the capabilities of the FCL Controller. While the results discussed below do not have statistical significance, they do provide insight on how the apparatus may be improved in future prototypes.

\section{A. Temperature and Humidity Sensor Validation}

Measurements were taken from both the ThermoPro indoor humidity and temperature monitor and the DHT22 sensor in two different environments and the results recorded, as shown in Table 5. For both temperature and relative humidity, higher values resulted in larger percent errors. For consistency between sets of data, it was decided the ThermoPro would be used to manually record the environmental conditions in all tests.

Table 5. Relative humidity and temperature readings made by the ThermoPro and DHT22 sensor in two separate environments.

\begin{tabular}{|c|c|c|c|c|c|c|}
\hline \multirow{2}{*}{ Environment } & \multicolumn{3}{|c|}{ Temp $\left({ }^{\circ} \mathbf{C}\right)$} & \multicolumn{3}{c|}{ RH (\%) } \\
\cline { 2 - 7 } & ThermoPro & DHT22 & \% Error & ThermoPro & DHT22 & $\%$ Error \\
\hline $\mathbf{1}$ & 22.2 & 22.2 & $0.00 \%$ & 40 & 43.3 & $8.25 \%$ \\
\hline $\mathbf{2}$ & 25.4 & 25.5 & $0.39 \%$ & 35 & 35.7 & $2.00 \%$ \\
\hline
\end{tabular}




\section{B. Continuous Drop Test I}

Continuous Drop Test I (CDTI) was conducted in the aforementioned large sized room using the default Controller parameters listed in Table 1, with Primitive 6 selected randomly. The big room did not have special measures taken to control the environmental conditions. Temperature and relative humidity were constant throughout the experiment at $22.30{ }^{\circ} \mathrm{C}$ and $51.90 \%$, respectively. Sixteen droplets dispensed before the cartridge ran out of fluid, prematurely ending the test. In this first test, it was unexpected for the sample to evaporate from the reservoir so quickly, so a cap had not been placed on the cartridge. This result led to the caps being developed and 3D printed for future tests.

The sixteen droplets were imaged, and none were found to have successfully formed webs. It was suspected that motor vibrations were the cause for lack of web formation, but it was acknowledged that the environmental conditions were not ideal. Since the single drop tests were expected to perform better than the continuous drop tests, the continuous drop tests were tabled until the viability of the system proven. Variation in the distance between droplets was noticed so attention was brought to the rotational accuracy of the stepper motor. 


\section{Stepper Motor Accuracy Validation}

The variation in distance between droplets in CDT1 led to an investigation of the positional accuracy of the stepper motor. The stepper motor accuracy validation test was repeated three times. Figure 31 shows the percent error of the theoretical position versus the measured position for each trial. The positional percent error of the motor was determined between successive measurements, so the results were not compounded. While the percent error was within the manufacturer's specification of $\pm 5 \%$ accuracy, this is not accurate enough to produce reliable alignment of the droplets with the objective lens.

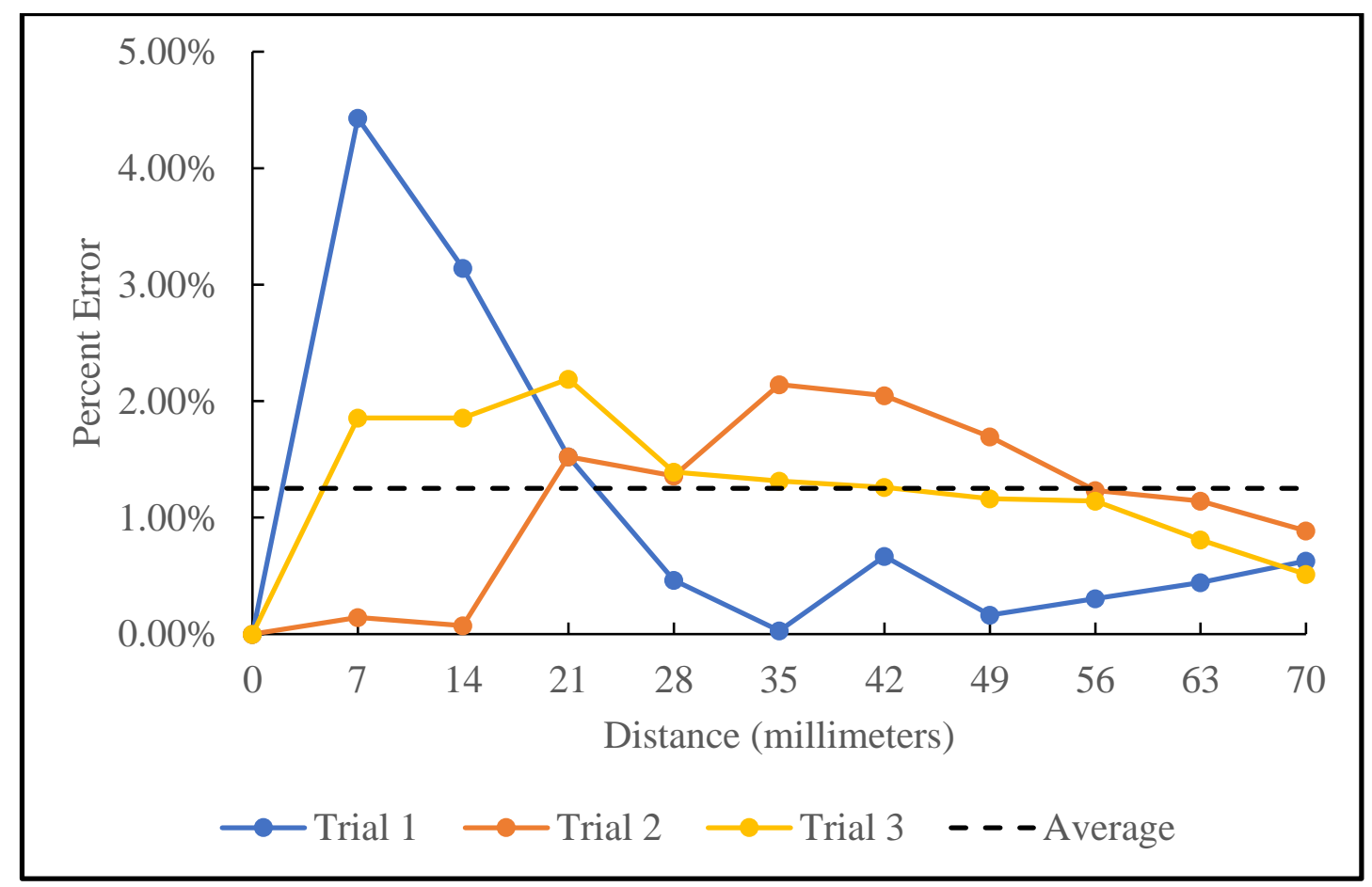

Figure 31. Positional error of stepper motor for 10 run commands. 


\section{Film Tension Test}

To ensure the maximum holding torque of the motor was not exceeded, a test was devised to measure the tension in the film so that the static torque applied to the motor could be calculated. The distance between the capstan and the supply reel holder was measured using a tape measure (Craftsman) to be 8.25 inches. The deflection was measured using digital calipers to be 0.185 inches. Using Equation 12, the tension in the film was calculated to be approximately 3.79 ounces.

The radius of the capstan was 0.75 inches. Equation 13 was used to calculate the film exerted 2.85 ounce-inches of torque on the motor. The motor's stall torque was rated 83.6 ounce-inches - making the motor significantly overpowered.

\section{E. Single Drop Test I}

The apparatus was moved to the small sized room into which a dehumidifier was placed to decrease the relative humidity. Single Drop Test I (SDTI) was conducted using the default Controller parameters listed in Table 1, with Primitive 2 selected randomly. Results for SDTI were recorded for each cartridge individually and are shown in in Figure 32. Filled markers indicate that the droplet resulted in the formation of a web pattern, while the unfilled markers indicate that a web pattern did not form. Ambient temperature increased throughout the test due to a warm body constantly monitoring the system in a small, thermally insulated room. As expected, the time to evaporate decreased with increasing temperature and decreasing humidity. It was not evident if chip degradation over the course of the test affected the droplet mass and, therefore, evaporation time of the droplets. 


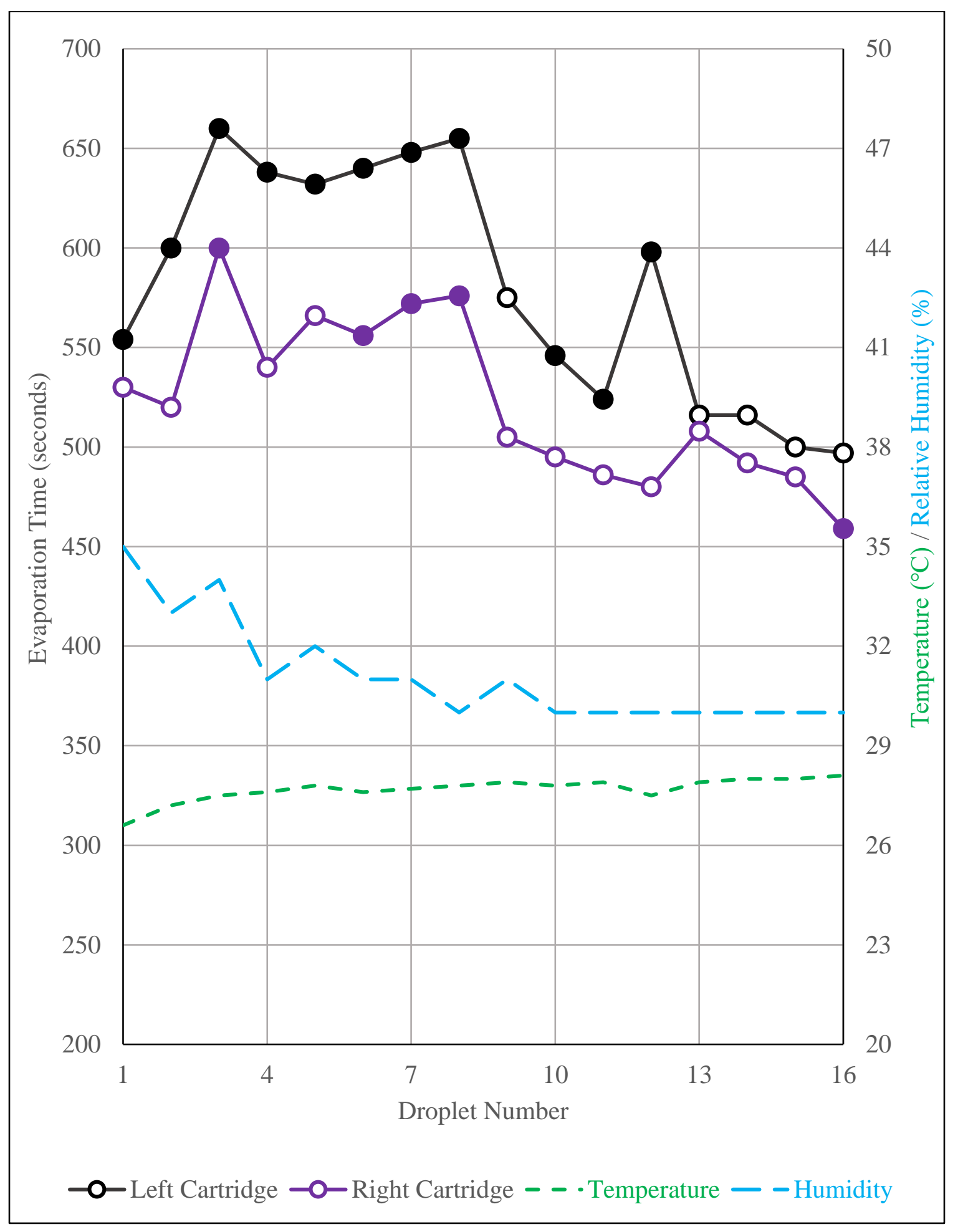

Figure 32. SDTI. Filled markers indicate a whiskey web pattern formed. Unfilled markers indicate whiskey web pattern did not form. 
Unsurprisingly, successful web formations decreased with increasing temperature. It appeared that for a relative humidity of $30 \%$, web formations were significantly hindered at $27.9^{\circ} \mathrm{C}$, as shown in Table 6. Previous studies demonstrated increased humidity resulted in greater web density, as shown in Figure 33, but the droplets took longer to evaporate (Carrithers et al., 2018). The previous studies also demonstrated that elevated ambient temperatures could prevent web formations but did not provide a conclusive critical temperature. The information gathered during SDTI should be used in the design of future temperature studies. It is apparent from Table 6 that the left cartridge had a higher success rate than the right cartridge, likely indicating a cleaner chip.

Table 6. SDTI web formation success rates.

\begin{tabular}{|c|c|c|}
\hline Success Rate & Left Cartridge & Right Cartridge \\
\hline Overall & $69 \%$ & $31 \%$ \\
\hline Below $27.9^{\circ} \mathrm{C}$ & $100 \%$ & $40 \%$ \\
\hline Above $27.9^{\circ} \mathrm{C}$ & $17 \%$ & $17 \%$ \\
\hline
\end{tabular}

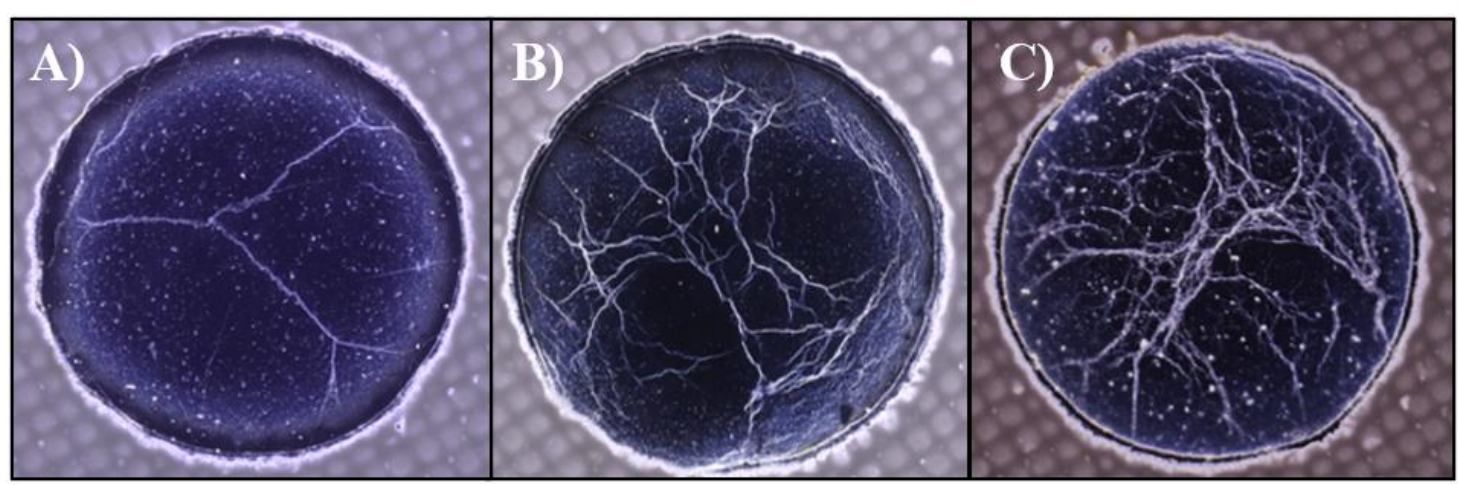

Figure 33. Effect of humidity on web formation. 2-millimeter wells. Woodford Reserve Double Oaked. A) 25\% RH, B) 45\% RH, C) 71\% RH. 


\section{F. Single Drop Test II}

The apparatus was moved to the medium sized room into which a dehumidifier was placed. The thermal equilibrium of the medium sized room occurred at a lower temperature while still maintaining a decreased relative humidity. Single Drop Test II (SDTII) was conducted using the same Controller parameters as SDT1. The cartridges were not cleaned between tests nor a different primitive chosen. Results are shown in Figure 34.

The left cartridge had a success rate of $75 \%$ and the right cartridge had a success rate of $44 \%$. The success rates in SDTII complement the success rates in SDTI in that the left cartridge again dispensed more droplets yielding successful web formations. There was an apparent downward trend in the evaporation time of droplets throughout the experiment, despite nearly constant environmental conditions. The decrease in evaporation time was likely due to decrease in droplet mass. 


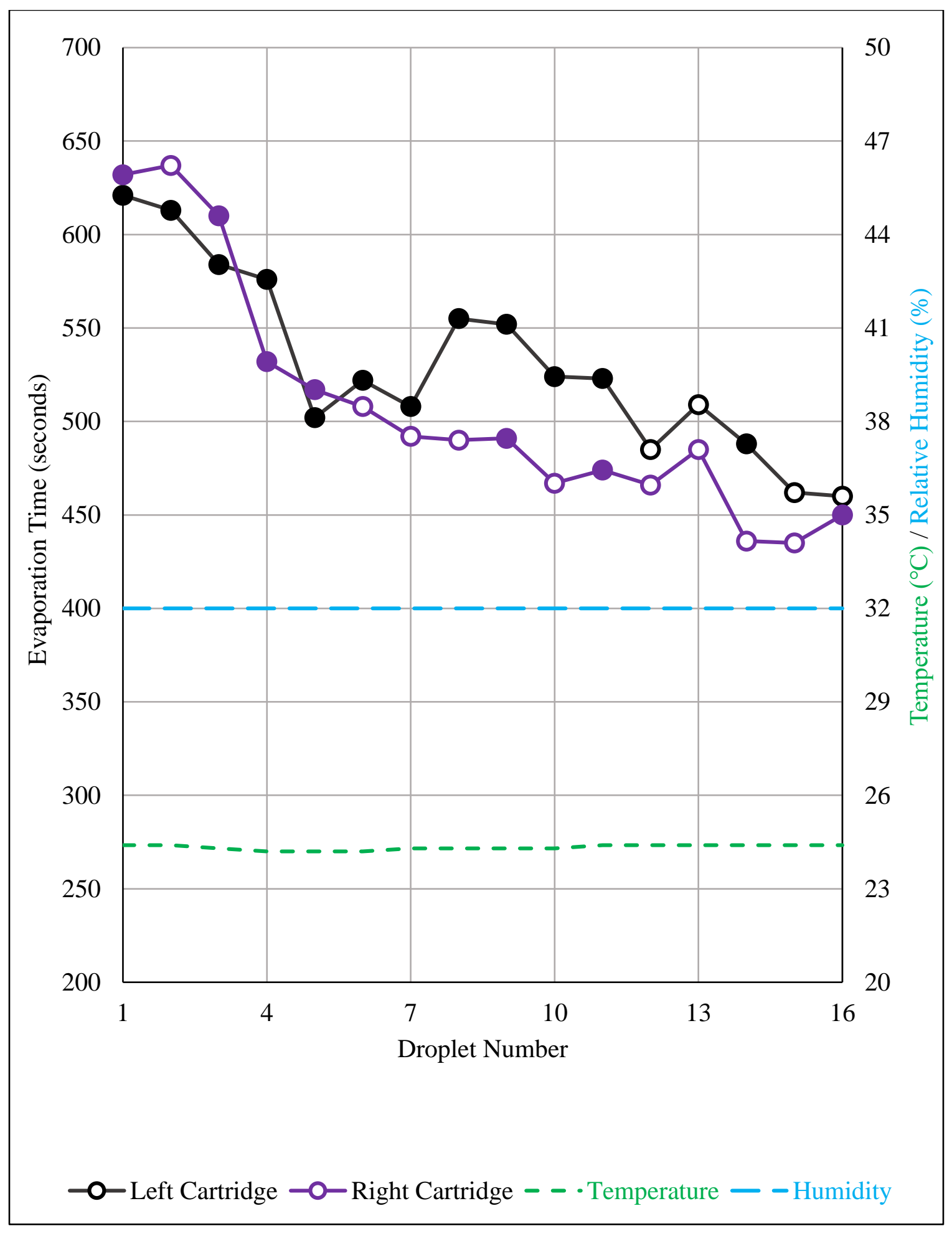

Figure 34. SDTII. Filled markers indicate a whiskey web pattern formed. Unfilled markers indicate whiskey web pattern did not form. 


\section{G. Cartridge Failure Analysis}

The decrease in droplet mass in SDTII indicated the printhead was failing, likely due to either kogation or channel blockage. Both cartridges were removed from the FCL Controller and examined for cause of failure.

Figure 35 depicts $2.5 x$ magnification and 20x magnification of the printhead chip on the left cartridge. It is apparent that most of the chip's nozzles are blackened, indicating kogation. At 20x it is also apparent that various contaminants are blocking the throat through which fluid travels to each nozzle chamber. When a contaminant impedes fluid from entering a chamber, the nozzle is unable to dispense full-sized (if any) droplets, reducing the overall drop mass. Typically, the felt and stainless-steel mesh filters would filter out most the contaminants found in the jetting fluid. However, as previously discussed, the contaminants that the filters are meant to remove are the same components from which whiskey webs are derived.

The chips were cleaned and reexamined. The printhead chip after cleaning of the left cartridge is shown in Figure 36. The blackening of the nozzle heaters was noticeably diminished after cleaning. However, from Figure 36B, it is apparent that there is still residual kogation on the heaters. For some nozzles, such as those in Primitives 2 and 4, the kogation was not removable. It is likely these primitives were used more heavily in preliminary testing. Large contaminants also remained stuck between the pillars and in the throat of several nozzles. It was expected that the droplets would continue to be inconsistent for the remainder of the experiments. For repeatable results, it is recommended the cartridges be replaced periodically, but more research is required to determine the frequency. 


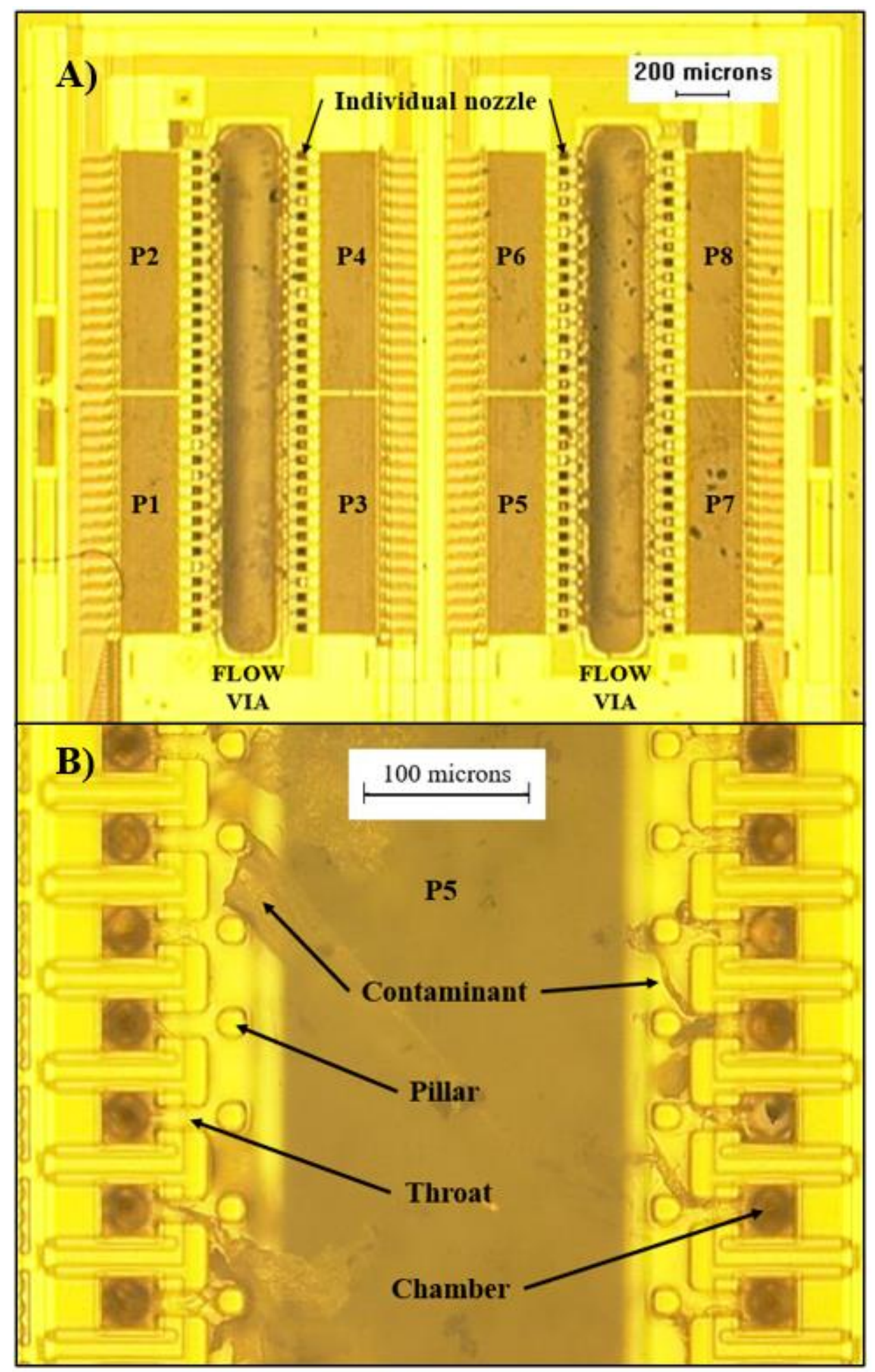

Figure 35. A) 2.5x magnification and B) 20x magnification of left cartridge chip after completion of SDTII. 


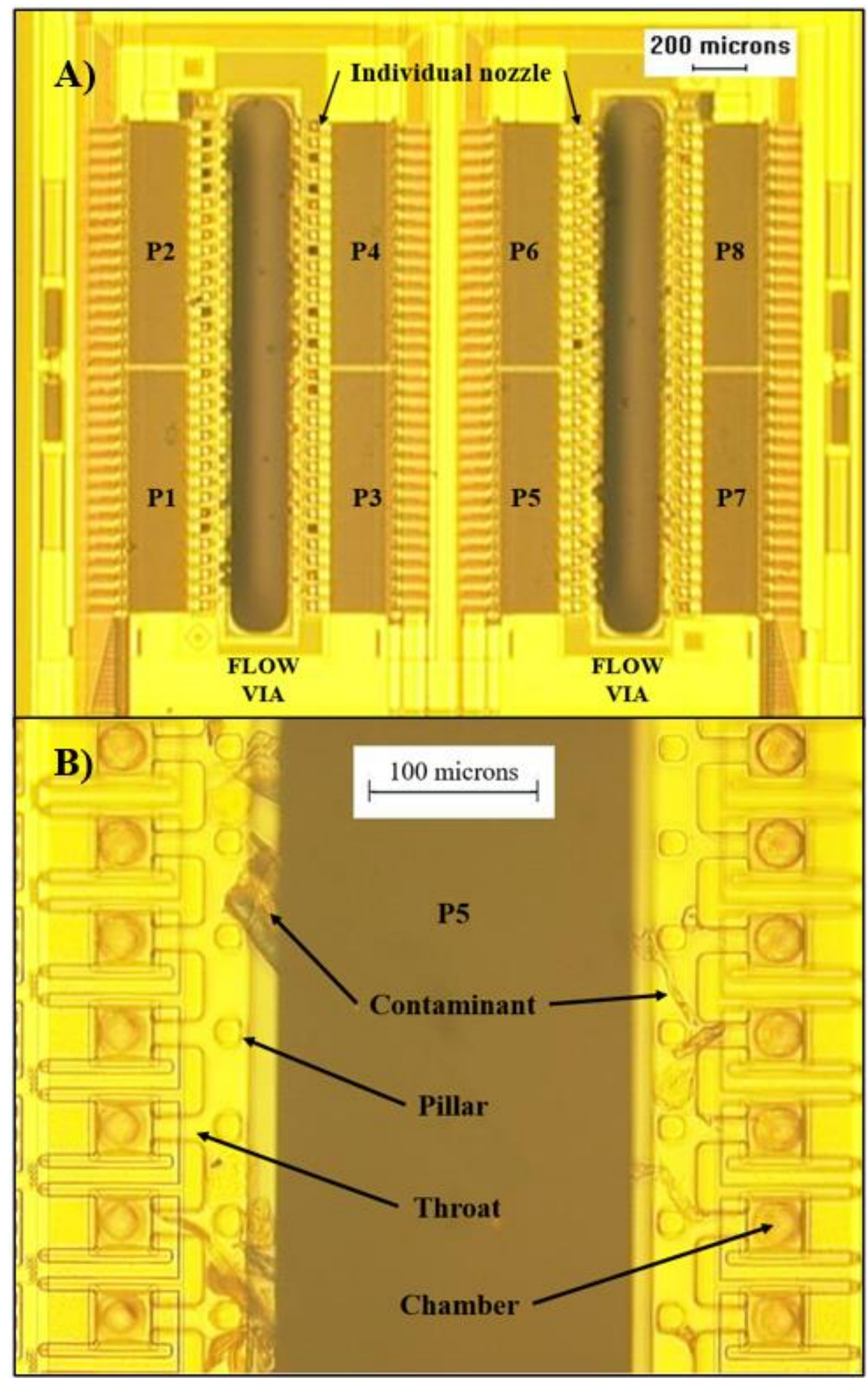

Figure 36. A) 2.5x magnification and B) 20x magnification of left cartridge chip after cleaning with 95\% ethanol and cotton swab. 


\section{$\underline{\text { H. Single Droplet Testing: Single Nozzle Variant }}$}

The Single Nozzle Variant of SDT was used to evaluate individual nozzle performance after cartridge failure analysis. Primitive 8 from the cleaned left cartridge was chosen for testing because it appeared to have the least amount of kogation and blockage after cleaning. Temperature was consistent at $24.4{ }^{\circ} \mathrm{C}$ and $\mathrm{RH}$ was constant at $34 \%$. Figure 37 demonstrates a positive correlation between a droplet's diameter and evaporation time, as expected. The droplets and their respective nozzles were sorted by ascending evaporation time. The web formation success rate was $88 \%$. Upon examination, it appeared droplet 10 failed due to contamination by dust or lint.

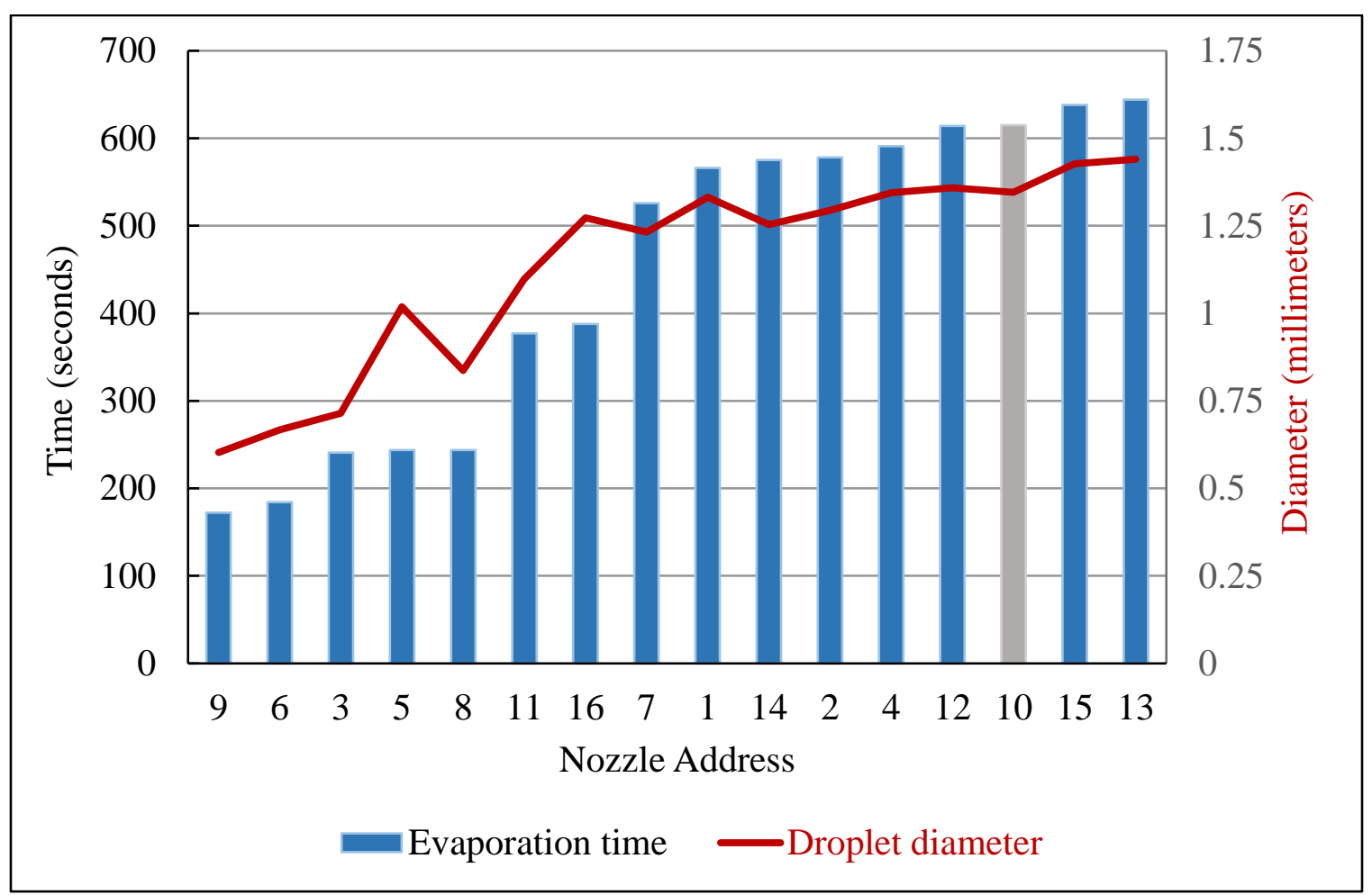

Figure 37. Evaporation time and diameter of droplets for each nozzle address in SNV.

Droplets were sorted by increasing evaporation time. Blue bars indicate a web pattern successfully formed while gray bars indicate a web pattern did not form. 
Assuming the droplet was hemispherical, and the outer ring of the evaporated droplet marks the initial pinning of the droplet's contact line, the volume of the droplet can be estimated using

$$
V=\frac{2 \pi r_{d r o p}^{3}}{3}
$$

where $r_{d r o p}$ is the radius of the evaporated droplet. The average droplet volume of the sixteen drops was 0.45 microliters with a standard deviation of 0.24 microliters. The minimum droplet volume was 0.06 microliters and the maximum droplet volume was 0.78 microliters. The webs produced in these tests could not be compared for consistency since the droplet sizes were inconsistent.

Although the droplet volumes were calculated assuming a hemispherical droplet, not all droplets left a circular profile. As shown in Figure 38, some of the more severely impeded nozzles, such as Nozzle 6, created satellites and irregularly shaped mother droplets. Ideally, dispensing from a single nozzle would result in almost perfectly round droplets, such as the one found in Figure $38 \mathrm{~B}$.

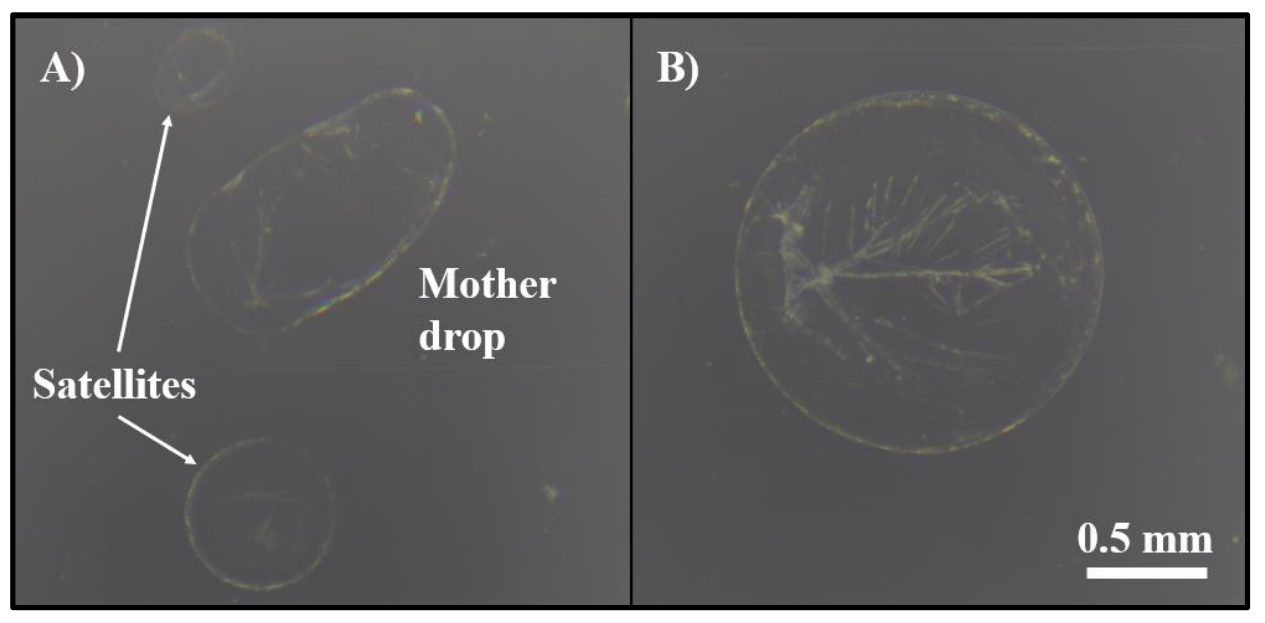

Figure 38. SNV evaporated droplets imaged for A) Nozzle 6, and B) Nozzle 13. 
The chip was reexamined after testing using the Nikon MM-60. From Figure 37, it was noted that the nozzles with the shortest evaporation time were Nozzles $9,6,3,5$, and 8, in order of increasing evaporation time. As shown in Figure 39, there is complete blockage between the pillar and throat of Nozzle 9. There is also a large contaminant blocking the throat of Nozzles 5 and 6 . Nozzle 8 does not show blockage, although the heater appears to be slightly darker than the those of the other nozzles, indicating kogation may be the cause of the decreased droplet mass.

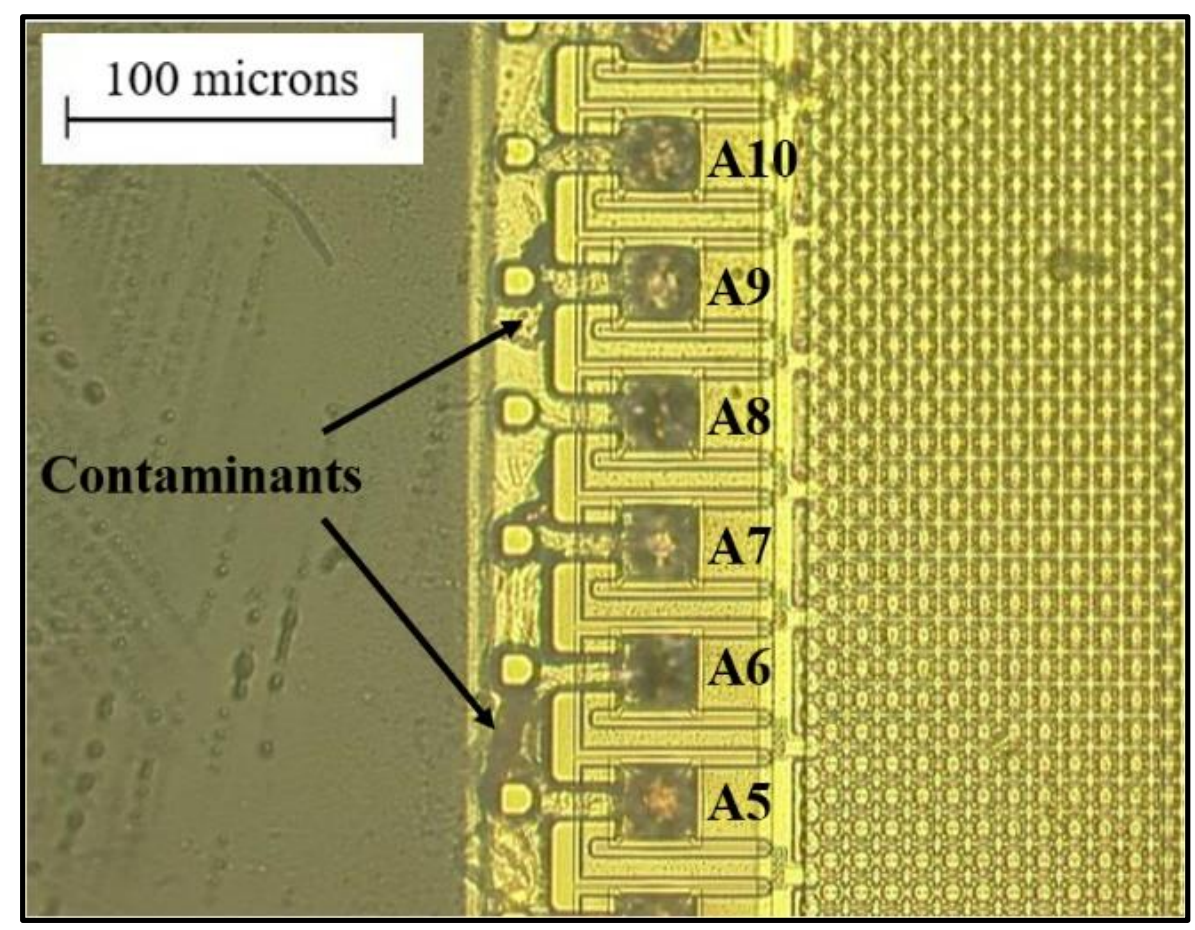

Figure 39. 20x magnification of Primitive 8, after SNV test. 


\section{$\underline{\text { I. Manual Drop Test }}$}

Temperature remained constant at $25.2^{\circ} \mathrm{C}$ and the relative humidity remained constant at $34 \%$ during testing. Figure 40 demonstrates, again, a positive correlation between the droplet diameter and evaporation time of the droplet. The average droplet volume was 1.13 microliters with a standard deviation of 0.17 microliters. The minimum droplet volume was 0.92 microliters and the maximum droplet volume was 1.31 microliters. Notably, every manually deposited droplet left a nearly circular profile once evaporated. The MDT resulted in $100 \%$ of the droplets forming web patterns.

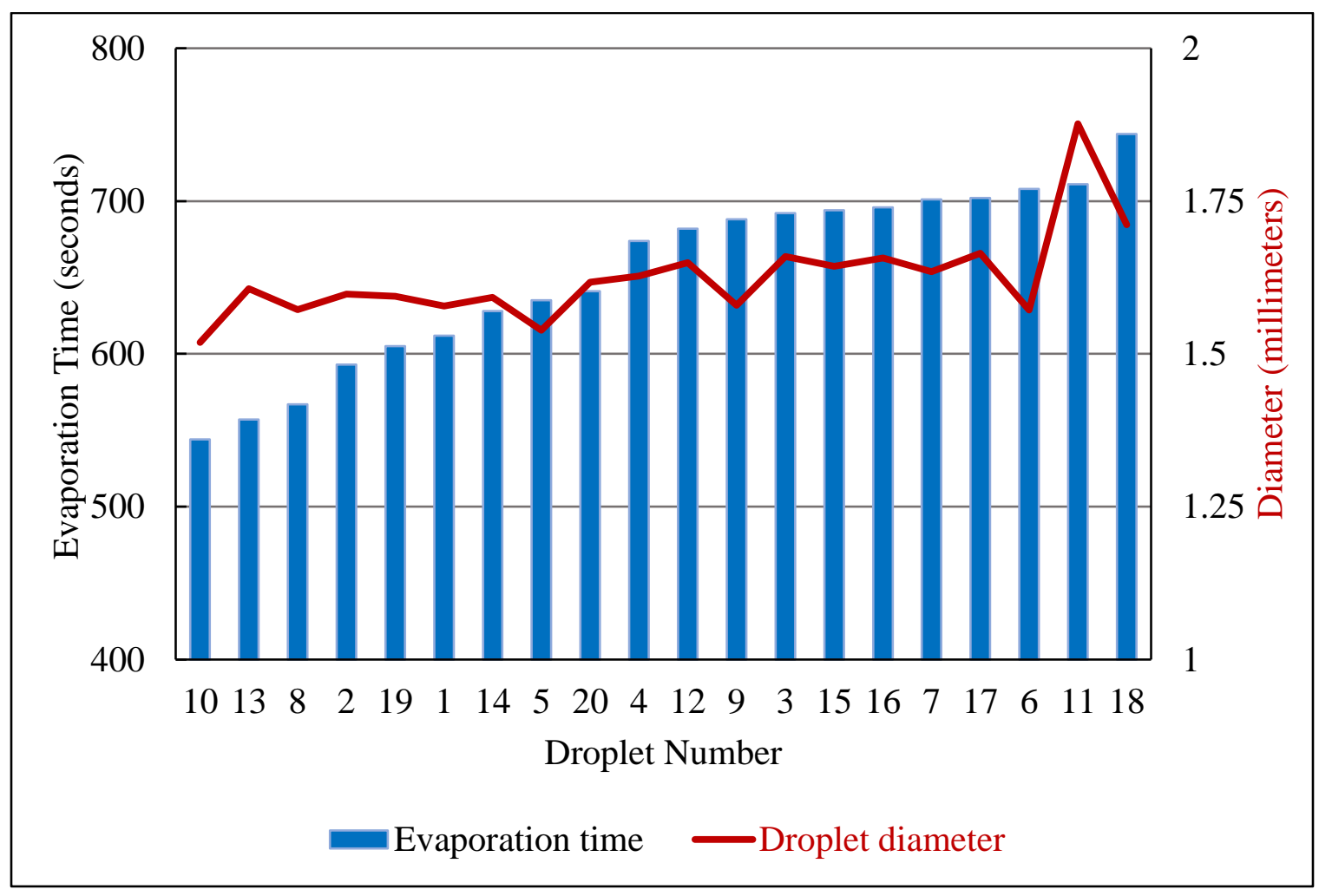

Figure 40. Evaporation time and diameter of manually deposited droplets. Droplets were sorted by increasing evaporation time. Blue bars indicate successful web formation. 


\section{$\underline{\text { J. Continuous Drop Test II }}$}

Lastly, a second continuous drop test (CDTII) was conducted in the new environmental conditions. The right cartridge, cleaned from printhead failure analysis, was used for testing. The test was conducted using the recommended parameters listed in Table 1, with Primitive 4 selected and an incremental address of 3. Using all sixteen nozzles had the drawback producing less circular droplet profiles but provided the benefit of more consistent droplet mass between droplets. The temperature was consistent at $24.5^{\circ} \mathrm{C}$ and the relative humidity was constant at $37 \%$. Evaporation times were not recorded, but droplet volumes were calculated using Equation 14 and compared across nozzles, shown in Figure 41. The success rate of web formation was 95\%.

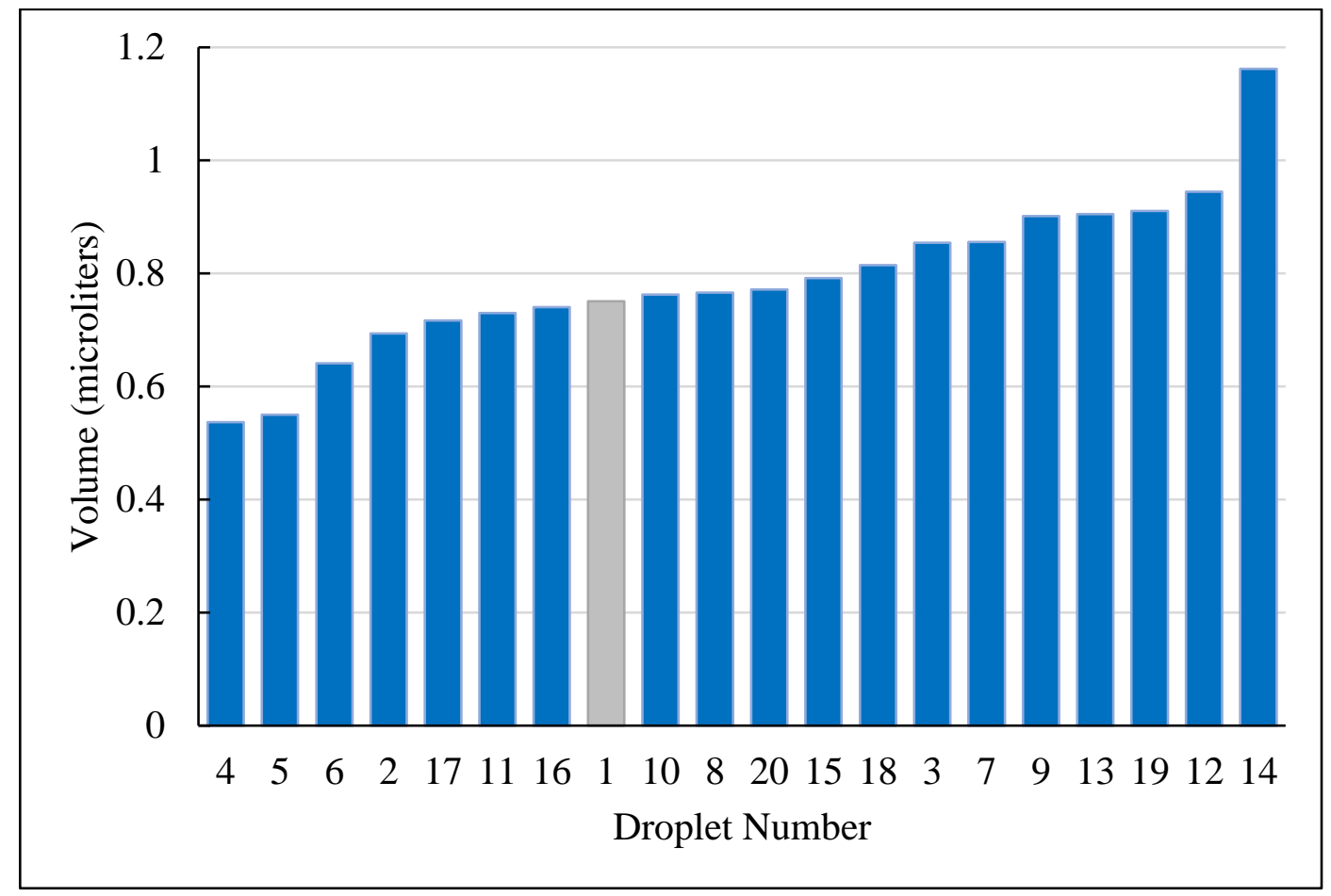

Figure 41. Droplet volumes of CTDII, sorted by increasing volume. Blue bars indicate a web pattern successfully formed while gray bars indicate a web pattern did not form. 
The average estimated droplet volume was 0.79 microliters with a standard deviation of 0.14 microliters. The minimum droplet volume was 0.75 microliters and the maximum droplet volume was 0.77 microliters. Of the twenty samples, only the first droplet did not form a web pattern. The first droplet's profile was that of a rounded square, while most droplets had an oblong profile, as depicted in Figure 42. The irregular shape of the first drop may be attributed to the printhead not being completely primed before it was fired. Since the droplet's profiles became more consistent after the initial droplet, it is likely that air bubbles were the cause of the chip not priming. To combat priming issues in the future, a sequence of code should be added to have the Controller dispense two or three test droplets that are not imaged to ensure fluid has filled all the chambers.

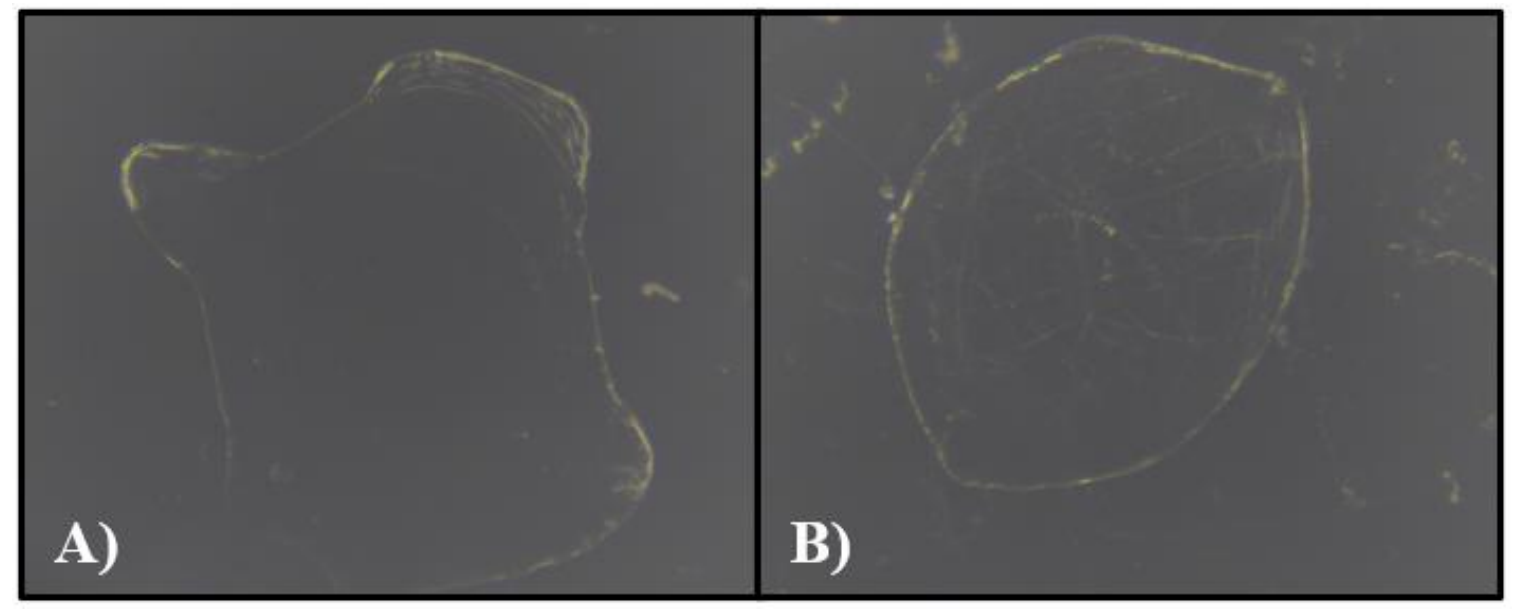

Figure 42. A) Droplet number 1 from CTDII test with a rounded square profile. B) Droplet number 10 from CTDII, representative of other droplets in the test. 


\section{$\underline{\text { K. Deposition Method Comparison }}$}

Four tests were compared to demonstrate the effect of dispensing methods on the consistency of the droplets: SDTII, SNV, CDTII, and MDT. Droplets representative of each batch of testing are shown in Figure 43. The Controller parameters for each of the automated tests are summarized in Table 7.

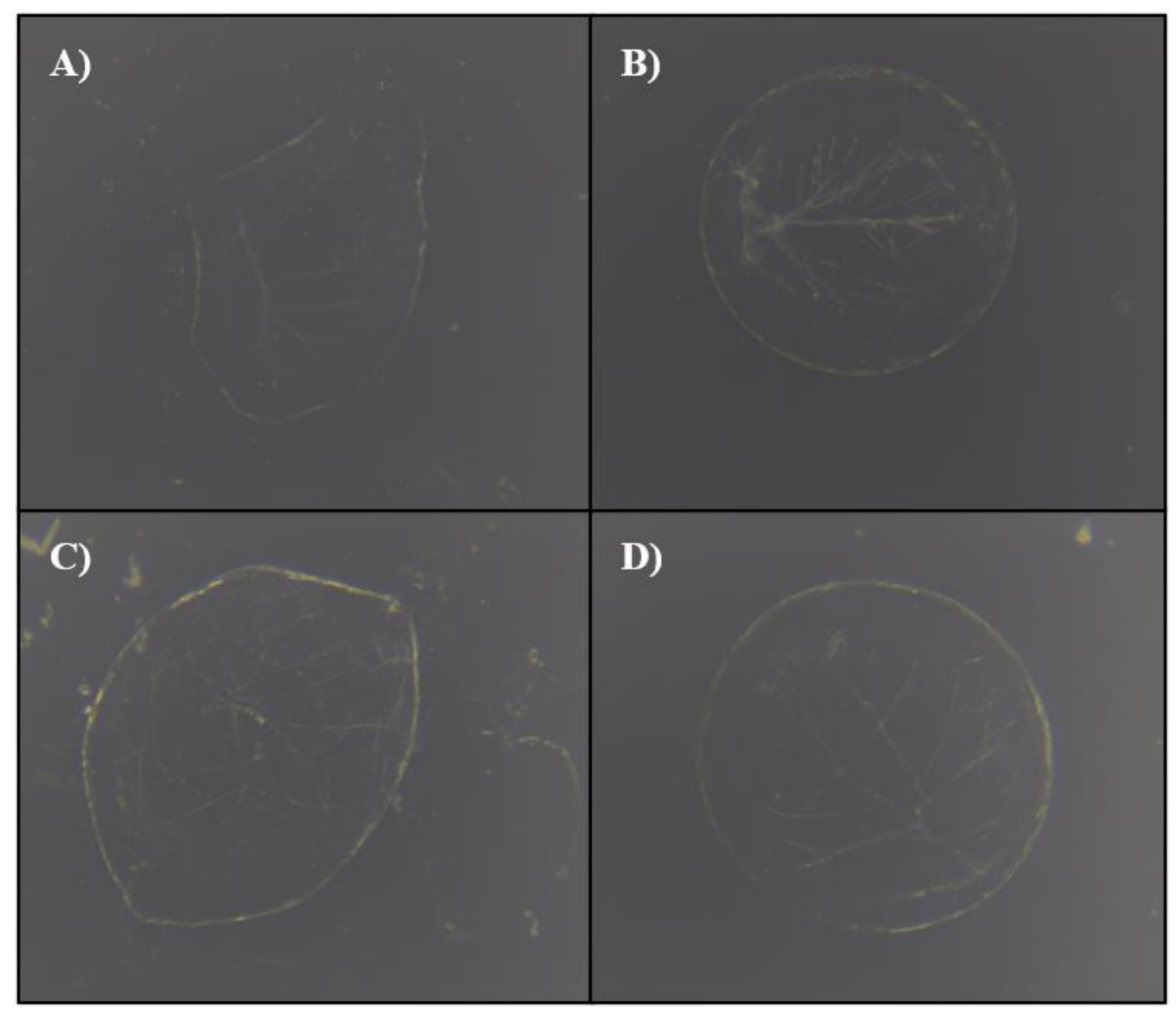

Figure 43. Droplet images representative of the tests:

A) SDTII, B) SNV, C) CDTII, D) MDT. 
Table 7. Controller parameters for SDTII, SNV, and CDTII.

\begin{tabular}{|l|c|c|c|}
\hline Parameter & SDTII & SNV & CDTII \\
\hline Dose $(\mu \mathrm{L})$ & 1 & 1 & 1 \\
\hline Preheat temp $\left({ }^{\circ} \mathrm{C}\right)$ & 45 & 40 & 40 \\
\hline Primitive & 2 & 8 & 4 \\
\hline Address & 26 & 13 & 10 \\
\hline Incremental Address & 3 & 1 & 3 \\
\hline Frequency $(\mathrm{Hz})$ & 1000 & 3000 & 3000 \\
\hline Voltage $(\mathrm{V})$ & 9 & 10 & 10 \\
\hline Pre-fire $(\mathrm{ns})$ & 100 & 150 & 150 \\
\hline Delay $(\mathrm{ns})$ & 300 & 1000 & 1000 \\
\hline Main fire $(\mathrm{ns})$ & 800 & 660 & 660 \\
\hline
\end{tabular}

The first and second single drop tests (SDTI and SDTII) were conducted using the default Controller parameters, which were not optimal for the jetting of this fluid. As shown Figure 43A, the typical droplet profile from these tests was highly irregular in shape. Comparing SDTII to CDTII illustrates how optimization of the FCL Controller's firing parameters can affect the regularity in shape of the droplets. Both SDTII and CDTII used an entire primitive for dispensing fluid, however CDTII used the settings recommended by FLTC. SDTII was conducted under different environmental conditions than CDTII, but this would not have affected the droplet's shape upon deposition. The firing parameters could likely be further optimized for diluted whiskies, but it is apparent that the default Controller settings should be avoided in future tests.

There is also considerable difference in the roundness of the droplet profile between CDTII and SNV. Because the nozzles of a primitive are arranged linearly, using all 16 nozzles results in the final droplet profile becoming elongated. Of the drops dispensed by the FCL Controller, the SNV droplet is most comparable in shape to the MDT. 
Table 8 provides a summarized comparison of the droplet size and consistency for manual deposition, continuous dropping using an entire primitive, and single dropping with a single nozzle.

Table 8. Comparison of droplet size and web formation success rate between the manual deposition technique, continuous dropping with an entire primitive, and single drop testing with a single nozzle.

\begin{tabular}{|c|c|c|c|c|}
\cline { 2 - 5 } \multicolumn{2}{c|}{} & MDT & CDTII & SNV \\
\hline \multirow{3}{*}{$\begin{array}{c}\text { Droplet Size } \\
\text { (microliters) }\end{array}$} & Minimum & 0.92 & 0.54 & 0.06 \\
\cline { 2 - 5 } & Maximum & 1.31 & 1.16 & 0.78 \\
\cline { 2 - 5 } & Average & 1.13 & 0.79 & 0.45 \\
\cline { 2 - 5 } & Std. Dev. & 0.17 & 0.14 & 0.24 \\
\hline \multicolumn{2}{|c|}{ Drop Profile } & Circle & Oblong & Circle \\
\hline \multicolumn{2}{|c|}{ Web formation } & $100 \%$ & $95 \%$ & $88 \%$ \\
\hline
\end{tabular}

CDTII, using an entire primitive, had a smaller standard deviation in droplet volume than the MDT despite suffering from kogation and blockage of the channels. For this chipset, the FCL Controller is capable of 24 picoliter resolution, so it is expected the Controller will far exceed the volume accuracy of the pipette when using a new cartridge. It is conceivable that a volume offset could be applied to the used cartridges to account for printhead degradation; although this method is not ideal if new cartridges are available.

Both the MDT and SNV resulted in circular droplet profiles. Although the MDT had a smaller standard deviation and average drop volume closer to desired, it is expected the SNV would have outperformed the pipette if new cartridges were available. 


\section{$\underline{\text { CHAPTER VII. SUMMARY }}$}

A reel-to-reel apparatus capable of automating the testing and imaging of whiskey webs was developed and fabricated. The system incorporated thermal inkjet technology which can provide much greater volumetric precision than traditional droplet dispensing techniques such as pipetting. Although more refinement is necessary, this system demonstrates the viability of a reel-to-reel assembly acting as the means for continuous, large-scale testing of whiskey webs. Methods for optimizing testing efficiency were provided so that the system can be scaled.

It has once again been shown that the formation of whiskey webs is sensitive to environmental conditions. While webs can form in a wide range of relative humidity, webs cannot form at some unknown critical temperature. In this study, the critical temperature at which webs could not form was $27.9^{\circ} \mathrm{C}$ at a relative humidity of $30 \%$.

Web formations can form despite vibrations of the substrate, although to what extent this affects the consistency of monolayer collapse mechanisms is unknown.

The components which form whiskey webs can be filtered out by standard inkjet cartridge filters. Due to the lack of filtering required to form whiskey webs, printheads are subject to increased degradation and ideally should be discarded between tests. Cleaning the chips provided some relief from kogation but did not remove contaminants blocking the channels to nozzles. 
The positional accuracy of the motor is critical to ensure evaporated droplets properly align with the camera objective for imaging. The current motor does not provide the accuracy necessary for reliable droplet imaging. The tension of the FEP film was measured and it was found the torque exerted by the film was significantly less than the stall torque of the motor.

Proper Controller firing parameters are necessary for regularly shaped and consistently sized droplets. Using a single nozzle results in a more circular droplet than using an entire primitive. Webs formed in circular droplet profiles can be more easily compared to other dispensing methods, such as pipettes. It is expected that for a new cartridge, SNV would provide the ideal conditions for consistently sized and shaped droplets - even outperforming manual deposition with pipettes. For a used cartridge, using all 16 nozzles of a primitive provided a better volumetric consistency compared to a single nozzle. While the firing parameters can likely be further optimized for whiskeys, the settings recommended by FLTC for a general solution of $25 \%$ ethanol is a good baseline for future tests.

Future investigations should be conducted to compare the consistency of web formations between thermal ink jetting and traditional pipetting techniques. This work has provided a necessary steppingstone towards mass data acquisition of whiskey webs to further enhance the statistical analysis of the unique features between each sample. 


\section{CHAPTER VIII. RECOMMENDATIONS}

First and foremost, the performance of the FCL Controller should be reevaluated with new cartridges once available. It is expected the single nozzle test will show improvement in droplet volume accuracy and standard deviation. To realize the effect of printhead chip degradation, tests should be conducted to determine how many droplets of whiskey sample can be dispensed with a single nozzle before decreased droplet volume occurs. It is suspected that even using new cartridges, using a full primitive for a single droplet will result in non-circular droplet profiles; nevertheless, this should also be confirmed. A hurdle to overcome in using only a single nozzle for each droplet is changing the nozzle address from the master code. Currently printer settings must be edited in the FCL program before testing commences.

Only 24 picoliter dispensing nozzles were found on the printheads used in this testing due to availability. Future studies could test printhead chipsets with larger nozzles and flow features, allowing for improved passing of web pertinent components. Finding a filter which can remove only the largest particles may also be valuable.

Temperature should be kept near room temperature. Although higher relative humidity has been shown to result in denser web formations, droplet evaporation time increases. A balance must be found to achieve consistent web patterns in reasonable amount of time. 
Although not discussed previously, the apparatus could be improved by implementing machined parts. Some of the larger 3D printed parts, such as the supply reel holder and supply reel cap, were prone to warpage. If the film were not carefully placed, it would twist as it was pulled by the capstan and pinch roller. The supply reel cap also seemed to suffer fatigue as result of applying pressure to the supply reel for braking, ultimately leading to reduced tension of the film throughout testing. Additional parts may be added to dampen vibrations in the film or help with alignment of the film with the objective lens.

It is recommended that a stepper motor with greater positional accuracy be chosen for future prototypes to ensure droplets are reliably imaged. There are stepper motors available with full-step resolutions of 0.9 degrees. A better full-step resolution equates to greater positional control of the film, allowing for a smaller distance between droplet centers and reducing the time of experiments. Using a stepper motor with a higher number of phases may also reduce the torque ripple and provide smoother movement. However, motors with more phases typically have a higher power consumption which equates to greater heat generation at low speeds.

Independent of motor precision, a motor with less torque should be considered for future prototypes. A stepper motor with a lower stall torque should be less expensive and likely reduce energy consumption, thus resulting in reduced heat generation while stationary. The rotor inertia of the current motor is $82 \mathrm{~g}-\mathrm{cm}^{2}$ and provides enough resistance that the film is held taut while the motor is turned off. A motor with less rotor inertia may need to be kept on continuously to provide the necessary tension in the film, meaning 
greater attention should be given to the motor's temperature. The torque applied to the motor by the film can be further reduced by using a smaller diameter capstan.

Vibration from the motor's acceleration and deceleration is also still a concern for the consistency of web patterns. It has not been proven that vibrations do not affect the formation of whiskey webs, only that the webs can form despite some vibration of the substrate. Acceleration of the motor can be adjusted by ramping the signal pulses from the Arduino. Future studies could include characterizing the effect of vibrations on web formation by vibrating glass slides while the droplets evaporate.

To aid in the positional error of the stepper motor, the distance between the printhead and camera objective, $l$, can be fine-tuned by mounting the FCL Controller to a horizontally mounted precision positioning slide and then adjusting the position of the Controller as needed. Figure 44 depicts two manually adjustable linear stages, either of which could be horizontally mounted to rightmost aluminum framing rail. Control of the distance $l$ would aid in optimizing the maximum number of droplets on the film.

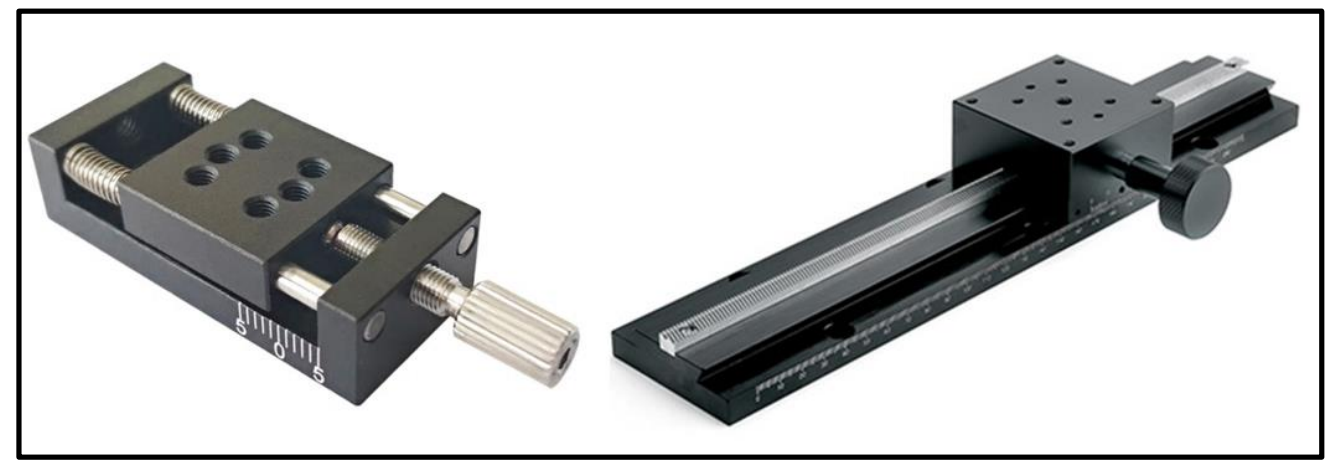

Figure 44. (Left) Screw driven, manually adjustable linear stage.

(Right) Rack and pinion manually adjustable linear stage. 
Since the filter was not used on the cartridges, there was no back pressure to prevent the printhead from drooling once the surface tension of the fluid is broken from the firing nozzles. Depending on the sample size, it may be necessary to provide a constant supply of the sample from some larger reservoir in large scale tests. This could likely be accomplished via feeding samples from a syringe pump to the cartridge at a constant rate.

Consideration should also be given to cleaning of the FEP film. The film was assumed clean in this study, although sometimes it was intentionally advanced to avoid oily residues. Either a standalone device could be used to clean the film, or a method could be incorporated into the current system thanks to the modularity of the optical breadboard.

If it is found in future testing that the droplets dispensed via the FCL Controller result in less consistent web patterns than manual deposition techniques, the methods and apparatus described herein could easily be converted to use a different dispensing technique. For example, a thin tube fed from a syringe pump could be mounted vertically several millimeters above the center of the film. Once a droplet forms at the tip of the tube, a motor could move the tube downwards so that the droplet is deposited onto the film. This method would not be affected by the filtering issues of the printheads but may present its own complications as fluid evaporates from the tube between droplets. 


\section{REFERENCES}

Abe, K., Suzuki, K., \& Citterio, D. (2008). Inkjet-Printed Microfluidic Multianalyte Chemical Sensing Paper. Analytical Chemistry, 80(18), 6928-6934. doi:10.1021/ac800604v

Allen, R. R., Meyer, J. D., \& Knight, W. R. (1985). Thermodynamics and Hydrodynamics of Thermal Ink Jets. Hewlett-Packard Journal, 36(5), 40.

Anyfantakis, M., Geng, Z., Morel, M., Rudiuk, S., \& Baigl, D. (2015). Modulation of the Coffee-Ring Effect in Particle/Surfactant Mixtures: the Importance of ParticleInterface Interactions. Langmuir, 31(14), 4113-4120. doi:10.1021/acs.langmuir.5b00453

Boone, K. (2019). Factors that Influence Whiskey Webs. Retrieved from

Bujake, J. E. (2007). Beverage Spirits, Distilled. In Kirk-Othmer Encyclopedia of Chemical Technology. New York.

Calvert, P. (2001). Inkjet Printing for Materials and Devices. Chemistry of Materials, 13(10), 3299-3305. doi:10.1021/cm0101632

Carrithers, A. D., Brown, M. J., Rashed, M. Z., Islam, S., Velev, O. D., \& Williams, S. J. (2020). Multiscale Self-Assembly of Distinctive Weblike Structures from Evaporated Drops of Dilute American Whiskeys. ACS Nano, 14(5), 5417-5425. doi:10.1021/acsnano.9b08984

Carrithers, A. D., Brown, M. J., \& Williams, S. J. (2018). Whiskey Webs: Analysis of Self-Assembled Microstructures from Evaporated Drops of Bourbon Whiskey. Paper presented at the American Physics Society Division of Fluid Dynamics, Atlanta, Georgia.

Chen, P.-H., Chen, W.-C., \& Chang, S. H. (1997). Bubble growth and ink ejection process of a thermal ink jet printhead. International Journal of Mechanical Sciences, 39(6), 683-695. doi:https://doi.org/10.1016/S0020-7403(96)00067-7

Coomes, P., \& Komstein, B. (2019). The Economic and Fiscal Impacts of the Distilling Industry in Kentucky. Kentucky Distillers' Association. 
Crampton, C. A., \& Tolman, L. M. (1908). A Study of the Changes Taking Place in Whiskey Stored in Wood. American Chemical Society, 30, 98-136.

Curak, M., Saranjam, N., \& Chandra, S. (2019). Colour variation in drying paint films. Progress in Organic Coatings, 136, 105173. doi:https://doi.org/10.1016/j.porgcoat.2019.06.019

Davison, M. W. (2020). Introduction to Microscope Objectives. Microscopy Basics. Retrieved from https://www.microscopyu.com/microscopy-basics/introduction-tomicroscope-objectives

Deegan, R. D., Bakajin, O., Dupont, T. F., Huber, G., Nagel, S. R., \& Witten, T. A. (1997). Capillary flow as the cause of ring stains from dried liquid drops. Nature, 389(6653), 827-829. doi:10.1038/39827

Girard, F., Antoni, M., Faure, S., \& Steinchen, A. (2008). Influence of heating temperature and relative humidity in the evaporation of pinned droplets. Colloids and Surfaces A: Physicochemical and Engineering Aspects, 323(1), 36-49. doi:https://doi.org/10.1016/j.colsurfa.2007.12.022

González-Gutiérrez, J., Pérez-Isidoro, R., \& Ruiz-Suárez, J. C. (2017). A technique based on droplet evaporation to recognize alcoholic drinks. Review of Scientific Instruments, 88(7), 074101. doi:10.1063/1.4991818

Hammond, D. L. (1984). Coping with Prior Invention. Hewlett-Packard Journal, 35(3), 44.

Hu, H., \& Larson, R. G. (2006). Marangoni Effect Reverses Coffee-Ring Depositions. The Journal of Physical Chemistry B, 110(14), 7090-7094. doi:10.1021/jp0609232

Interpol. (2017). Over $€ 100$ million worth of fake food and drinks seized in latest Europol-INTERPOL operation [Press release]. Retrieved from https://www.europol.europa.eu/newsroom/news/over-€100-million-worth-offake-food-and-drinks-seized-in-latest-europol-interpol-operation J. R. Piggott, R. Sharp, \& Duncan, R. E. B. (1989). The Science and Technology of Whiskies. New York: John Wiley \& Sons. 
Jr., R. A. F. (2016). The Whiskey Machine: Nanofactory-Based Replication of Fine Spirits and Other Alcohol-Based Beverages (IMM Report No. 47). Retrieved from http://www.imm.org/Reports/rep047.pdf

Kim, H., Boulogne, F., Um, E., Jacobi, I., Button, E., \& Stone, H. A. (2016). Controlled Uniform Coating from the Interplay of Marangoni Flows and Surface-Adsorbed Macromolecules. Physical Review Letters, 116(12), 124501. doi:10.1103/PhysRevLett.116.124501

Kjellander, B. K. C., Wiljan, T. T. S., Anthony, J. E., \& Gelinck, G. H. (2010). Inkjet printing of TIPS-PEN on soluble polymer insulating films: a route to highperformance thin-film transistors. Advanced Materials, 22(1521-4095 (Electronic)), 4612-4616.

Kuang, M., Wang, L., \& Song, Y. (2014). Controllable Printing Droplets for HighResolution Patterns. Advanced Materials, 26(40), 6950-6958. doi:10.1002/adma.201305416

Liao, H., Zhu, Z., \& Peng, Y. (2018). Potential Utility of Retinal Imaging for Alzheimer's Disease: A Review. Frontiers in Aging Neuroscience, 10(188). doi:10.3389/fnagi.2018.00188

Liebmann, A. J., \& Rosenblatt, M. (1943). Changes in Whisky While Maturing. Industrial and Engineering Chemistry, 35, 994-1002.

Mampallil, D., \& Eral, H. B. (2018). A review on suppression and utilization of the coffee-ring effect. Advances in Colloid and Interface Science, 252, 38-54. doi:https://doi.org/10.1016/j.cis.2017.12.008

Mortimer Abramowitz, M. W. D. (2020). Mechanical Tube Length. Anatomy of the Microscope. Retrieved from https://www.olympuslifescience.com/en/microscope-resource/primer/anatomy/tubelength/

Mueller, J. P., \& Massaron, L. (2016). Machine Learning for Dummies (K. Mohr Ed.). Hoboken, New Jersey: John Wiley \& Sons Inc.

Poulard, C., \& Damman, P. (2007). Control of spreading and drying of a polymer solution from Marangoni flows. Europhysics Letters (EPL), 80(6), 64001. doi:10.1209/0295-5075/80/64001 
Prince, S. J. D. (2012). Computer Vision : Models, Learning, and Inference. Cambridge, UNKNOWN: Cambridge University Press.

Russell, I. (2003). Whisky: Technology, Production, and Marketing (G. S. Charles W. Bamforth, Russell David Ed. 1 ed.): Academic Press.

Sefiane, K., Tadrist, L., \& Douglas, M. (2003). Experimental study of evaporating waterethanol mixture sessile drop: influence of concentration. International Journal of Heat and Mass Transfer, 46(23), 4527-4534. doi:https://doi.org/10.1016/S00179310(03)00267-9

Shirota, K., Shioya, M., Suga, Y., \& Eida, T. (1993). Kogation of Inorganic Impurities in Bubble Jet Ink. Paper presented at the IS\&T's Ninth International Congress on Advances in Non-Impact Printing Technologies, Yokohama, Japan. Www.imaging.org/site/PDFS/Papers/1996/RP-0-66/2161.pdf

Williams, M. A., McGowan, A. J., Cardwell, C. R., Cheung, C. Y., Craig, D., Passmore, P., . . McKay, G. J. (2015). Retinal microvascular network attenuation in Alzheimer's disease. Alzheimer's \& Dementia: Diagnosis, Assessment \& Disease Monitoring, 1(2), 229-235. doi:10.1016/j.dadm.2015.04.001

Williams, S. J., Brown, M. J., \& Carrithers, A. D. (2019). Whiskey webs: Microscale "fingerprints" of bourbon whiskey. Physical Review Fluids, 4(10), 100511. doi:https://doi.org/10.1103/PhysRevFluids.4.100511

Yunker, P. J., Still, T., Lohr, M. A., \& Yodh, A. G. (2011). Suppression of the coffeering effect by shape-dependent capillary interactions. Nature, 476(7360), 308311. doi:10.1038/nature 10344 
$\underline{\text { APPENDIX A }}$

Full text of:

Multiscale Self-Assembly of Distinctive Weblike Structures from Evaporated Drops of

Dilute American Whiskeys

Published in ACS Nano 2020, 14, 5, 5417-5425

DOI: https://pubs.acs.org/doi/full/10.1021/acsnano.9b08984
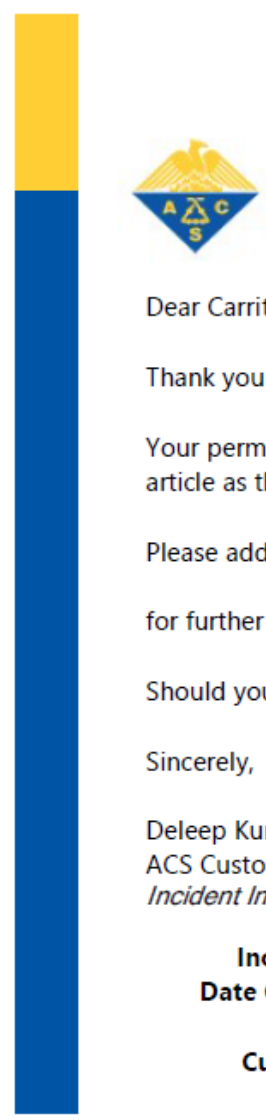

\section{ACS}

Chemistry for Life

Dear Carrithers,Adam David,

Thank you for contacting ACS Publications Help Desk.

Your permission requested is granted and there is no fee for this reuse. In your planned reuse, you must cite the ACS article as the source,

Please add this direct link https://pubs.acs.org/doi/full/10.1021/acsnano.9b08984

for further permissions related to the material excerpted should be directed to the ACS.

Should you have any questions or need further assistance, please feel free to contact me.

Sincerely,

Deleep Kumar

ACS Customer Services \& Information

Incident Information:

Incident \#: 3649233

Date Created: 2020-07-02T09:08:32

Priority: 3

Customer: Carrithers,Adam David

Title: Permission to use article in thesis 


\section{Multiscale Self-Assembly of Distinctive Weblike Structures from Evaporated Drops of Dilute American Whiskeys}

Adam D. Carrithers, ${ }^{\S}$ Martin J. Brown, VI, ${ }^{\S}$ Mohamed Z. Rashed, Sabina Islam, Orlin D. Velev, and Stuart J. Williams*

Cite This: ACS Nano 2020, 14, 5417-5425

Read Online

\begin{tabular}{|c|c|c|c|}
\hline ACCESSI & |lll Metrics \& More & 므 Article Recommendations & (a) Supporting Information \\
\hline
\end{tabular}

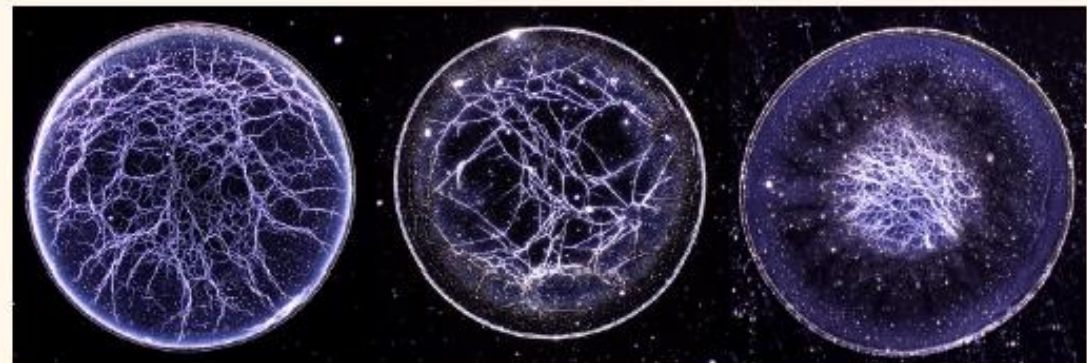

ABSTRACT: When a sessile droplet of a complex mixture evaporates, its nonvolatile components may deposit into various patterns. One such phenomena, the coffee ring effect, has been a topic of interest for several decades. Here, we identify what we believe to be a fascinating phenomenon of droplet pattern deposition for another well-known beverage-what we have termed a "whiskey web". Nanoscale agglomerates were generated in diluted American whiskeys (20-25\% alcohol by volume), which later stratified as microwebs on the liquid-air interface during evaporation. The web's strandlike features result from monolayer collapse, and the resulting pattern is a function of the intrinsic molecular constituents of the whiskey. Data suggest that, for our conditions (diluted $1.0 \mu \mathrm{L}$ drops evaporated on cleaned glass substrates), whiskey webs were unique to diluted American whiskey; however, similar structures were generated with other whiskeys under different conditions. Further, each product forms their own distinct pattem, demonstrating that this phenomenon could be used for sample analysis and counterfeit identification.

KEYWORDS: self-assembly, colloids, surface monolayers, Marangoni flow, droplet evaporation, whiskey

\begin{abstract}
A n evaporating droplet containing nonvolatile solutes leaves a deposit whose form is dependent on the intrinsic properties of the liquid,,$^{1,2}$ the nature of the solutes or particles, ${ }^{3,4}$ and the environmental conditions under which evaporation takes place. ${ }^{5,6}$ Understanding the deposition of such nonvolatile solutes is critical for engineering of coating and patterning processes. ${ }^{7}$ One common phenomenon, which can arise in these coating processes, is the coffee ring deposition effect where solute particles are transported to the pinned contact line via capillary flow. ${ }^{8}$ Suppression of the coffee ring effect has been extensively studied with various liquids," including Scotch whisky, which yielded nearly uniform particle deposition. ${ }^{10}$
\end{abstract}

Here, we demonstrate a self-assembled structure resulting from the evaporation of a volatile sessile drop of American whiskeys - depositing hierarchical weblike patterns that we have termed a "whiskey web". ${ }^{11}$ These webbed patterns were formed by evaporating a $1.0 \mu \mathrm{L}$ droplet of diluted American whiskey onto a clean glass coverslip. Results herein will

Received: November 12, 2019

Accepted: March 9, 2020

Published: March 25, 2020

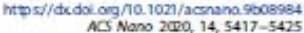



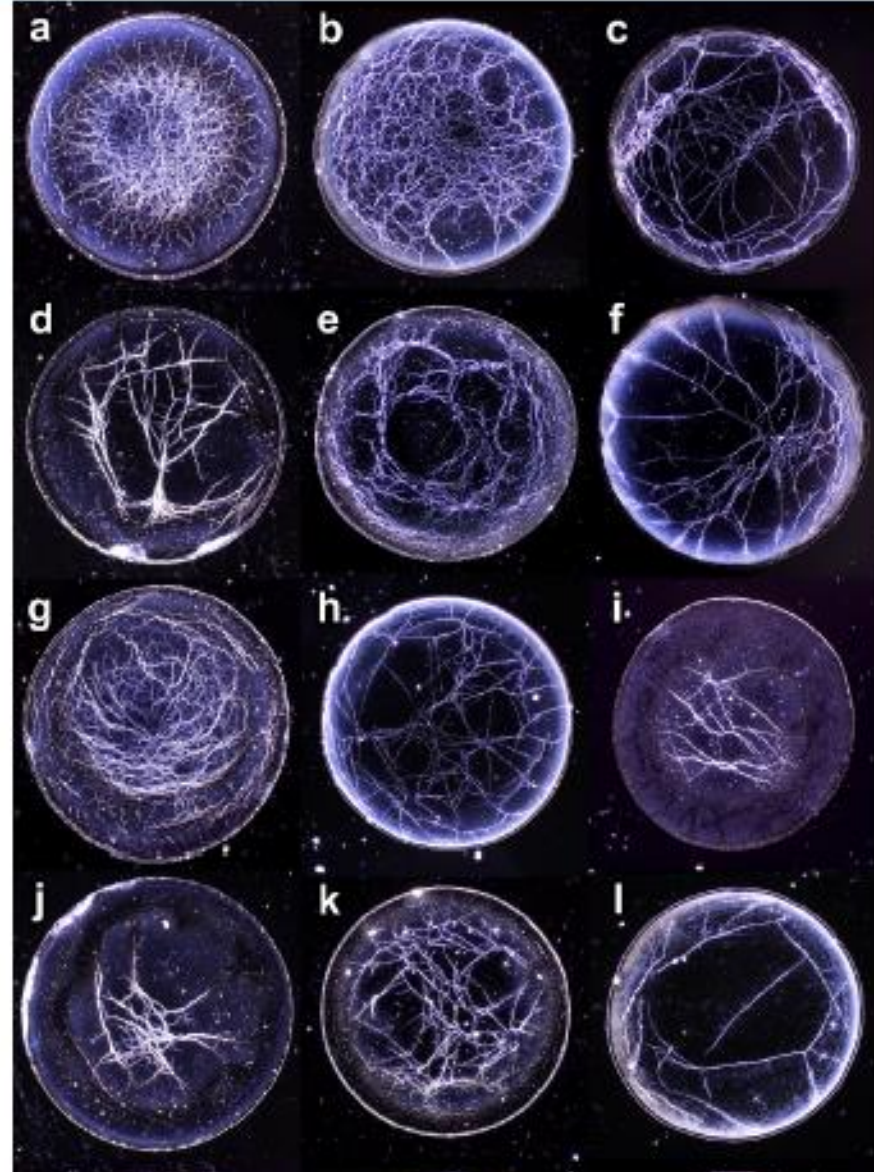

k
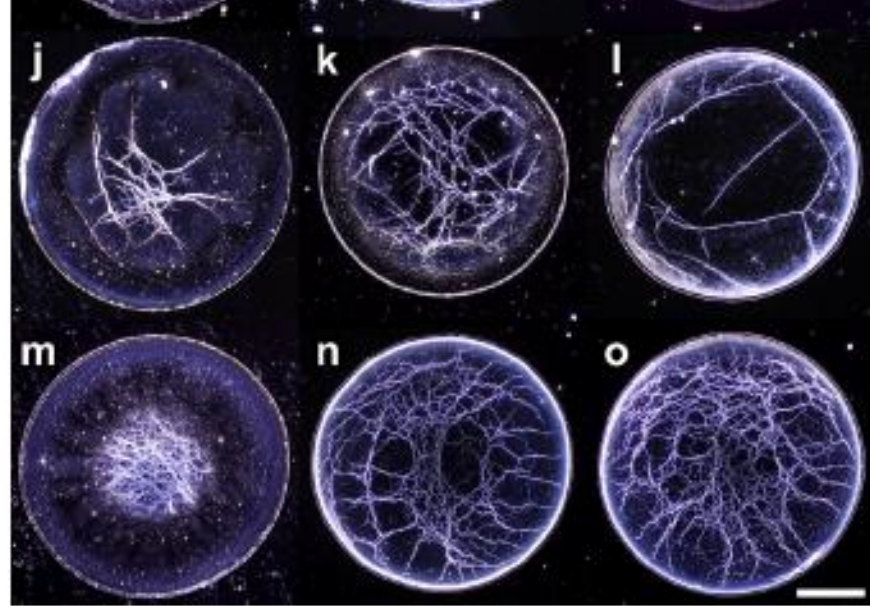

Figure 1. Examples of the surprisingly diverse "whiskey web" patterns. The pattems, approximately 2 mm in diameter, were formed by drying droplets from various off-the-shelf whiskey products diluted to $20-25 \%$ alcohol by volume. Scale bar is $0.5 \mathrm{~mm}$. Refer to Table $\$ 1$ in the Supporting Information for sample identification. Adapted with permission under a Creative Commons Attribution-NonCommercil 4.0 International License from ref 11, https1//doi.org/10.1103/APS.DFD.2018.GFM.P0002. Copyright 2018 Stuart J. Willims

demonstrate that these weblike structures are monolayers of water-insoluble chemical constituents that collapsed at the interface of the sessile droplet. Traditionally, Langmuir monolayers have been studied to gain insight into the behavior of biological membranes ${ }^{12}$ to develop thin films, ${ }^{13}$ or to understand structural and phase transitions. ${ }^{14-16}$ Inadvertently, this work demonstrated not only that such structures are created during droplet evaporation, as one might expect for the analogous monolayer compression experiment, ${ }^{17-19}$ but also that the resulting pattem serves as chemical "fingerprints" of the liquid. Figure I shows a selection of qualitatively repeatable patterns generated from evaporated $1.0 \mu \mathrm{L}$ drops of diluted
(20-25\% alcohol by volume) American whiskey deposited on coverslip glass.

The chemical composition of the whiskeys has an obvious impact on monolayer composition and connectivity, thus resulting in different patterns. The folds themselves can resemble "twisted ribbons", and in certain cases, monolayer collapse leads to the formation of vesicles. ${ }^{1420}$ The collapse of the monolayers is further influenced by interfacial intermolecular interactions, induding alkyl hain length, headgroup ionization, strength of interfacial hydrogen bonding, temperature, and compression rate. ${ }^{21}$ Its heterogeneity, induding contaminants plays a role in the formation of the collapsed 
structure. ${ }^{22,23}$ The mechanism of collapse also depends on the elasticity and cohesiveness of the monolayer. ${ }^{24}$ Previous reports with relevance to this study have shown that whiskey inherently contains chemicals that facilitate the generation of interfacial monolayers, ${ }^{25}$ and dilution of whiskey with water facilitates the transport of chemicals to the interface ${ }^{26}$

American whiskey is a spirit distilled at less than $95 \%$ alcohol by volume (ABV) from a fermented mash of grain and is bottled at no less than $40 \% \mathrm{ABV}^{27}$ Various forms of American whiskey exist (i.e, bourbon whiskey, rye whiskey, wheat whiskey, etc.) with the defining characteristic being the mixture of grains used in the mashing process. ${ }^{23}$ During fermentation, secondary products, known as congeners, are formed by side reactions and determine most of the organoleptic qualities of the final product. The congeners include phenols, aromatics, esters, aldehydes, higher alcohols, and trace substances. ${ }^{29}$ After distillation, the product could be stored in a charred new oak barrel for at least two years but is typically stored for four years or longer. During the storage period, complex wood constituents are extracted by the liquid, and reactions occur between various organic substances, resulting in maturation of the product. Maturation of whiskey increases the concentration of acids, esters, and dissolved solids ${ }^{30,31}$ American whiskey differentiates itself from other whiskeys in that maturation occurs in new charred oak containers. The amount of solids contained in American whiskey is greater than whiskeys aged in reused containers $(1.8 \mathrm{~g} / \mathrm{L}$ for straight bourbon whiskey compared to $0.97 \mathrm{~g} / \mathrm{L}$ for Canadian whiskeys and 1.27 for Scotch). ${ }^{32}$ Whiskeys derived from new charred oak barrels have relatively larger concentrations of water-insoluble content. $^{31}$ Further, bourbon whiskey has typically been described as more resinous compared to other whiskeys, with the foam from the former being more oily and remaining much longer than that in the latter.

Many of the organic components contained in whiskey are amphipathic and alcohol-soluble. ${ }^{26}$ The dilution of a whiskey with water results in the aggregation of the amphipathic molecules into nanoscale agglomerates. This can be demonstrated by diluting the whiskey with water, while stirning the solution and shining a laser through the side of the container (Figure 2). Due to the Tyndall effect, ${ }^{33}$ the scattered light from the laser is brightest at a dilution of $20-30 \% \mathrm{ABV}$, where agglomerates are largest in size and/or concentration. This visualization effect is reduced at higher alcohol concentrations due to the alcohol-soluble nature of the constituents. These

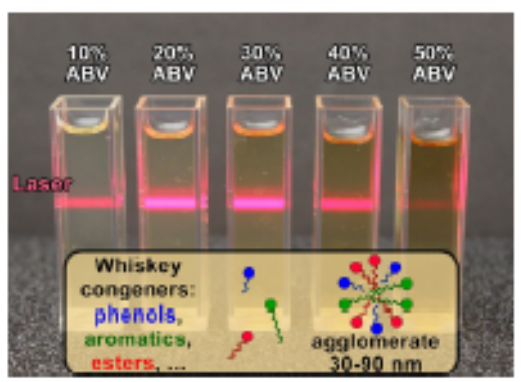

Figure 2. Emergence of colloidal systems in diluted whiskeys. Demonstration of the Tyndall effect, where a laser beam visualizes the suspended agdomerates in various dilutions of a bourbon whiskey. qualitative observations guided droplet evaporation expenments, which were conducted at $20-25 \% \mathrm{ABV}$.

\section{RESULTS AND DISCUSSION}

First, we investigated whether such structures deposited on the substrate or formed on the liquid-air interface. A time-lapse of monolayer formation visualized with dark-field phase contrast is shown in Figure 3 (with an accompanying Movie $\mathrm{S1}$,

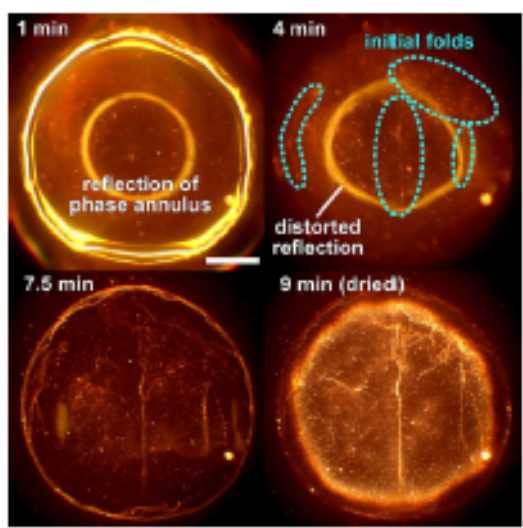

Figure 3. Time-lapse of a $0.75 \mu \mathrm{L}$ drop of diluted ( $25 \% \mathrm{ABV}$ ) bourbon whiskey evaporating on the surface of an ITO-coated glass slide under ambient conditions. Evaporation is observed with an upright microscope using a dark-field phase annulus. At 4 min, monolayer folds were observed that subsequently impact the topography of the droplet. Additional folds were created as the droplet evaporates $(7.5 \mathrm{~min})$, and these features are highlighted once the liquid has completely evaporated $(9 \mathrm{~min})$. Scale bar is 0.5 mm

Supporting Information). Through manual focus adjustment, it was confirmed that the structures were at the liquid-air interface, suggestive of monolayer formation and collapse. The initial image (Figure 3, $1 \mathrm{~min}$ ) was relatively axisymmetric, evidence of the circular reflection of the phase annulus. However, the reflection became distorted as the monolayer collapsed (Figure 3, $4 \mathrm{~min}$ ). The monolayer continued to collapse across the droplet surface area, and these features were more dearly visible once the fluid completely evaporated (Figure 3, $9 \mathrm{~min}$ ).

A side-view time-lapse showing the evaporation of a diluted bourbon whiskey drop is shown in Figure 4, confirming interfacial distortion during evaporation. During evaporation, the monolayer was exposed to stress due to the decreasing surface area, eventually causing it to collapse when exposed to a surface pressure greater than the equilibrium spreading pressure. ${ }^{22}$ The overlapping of microcrystalline, inhomogeneous textures occurs during continuous overcompression. ${ }^{21}$ Assuming a spherical cap and a base diameter of $2.0 \mathrm{~mm}$, the surface area of a $1.0 \mu \mathrm{L}$ pinned droplet will reduce by approximately $33 \%$ during evaporation. Further, the presence of a rigid monolayer will dismpt sessile evaporation, ${ }^{3+}$ induding significantly reducing the rate of evaporation ${ }^{35,36}$

Figure 5 illustrates how whiskey web structures were created. First, when a droplet of a diluted whiskey is initially placed onto the substrate, agglomerates are driven to the surface of the droplet through solutal Marangoni flows ${ }^{37}$ characterized by 


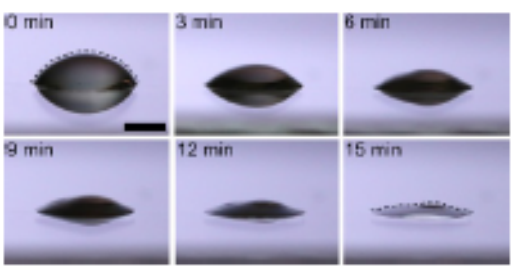

Figure 4. Series of images illustrating the evaporation of $2.5 \mu \mathrm{L}$ drop of diluted ( $25 \%$ ABV) bourbon whiskey on the surface of an rro-coated glass slide under ambient conditions. A distorted (ie., nonarisymmetric) surface profile is observed, suggesting inhomogeneous mechanical properties at the liquid-air interface. Scale bar is $1.0 \mathrm{~mm}$. Refer to Table $\$ 1$ in the Supporting Information for sample identification.

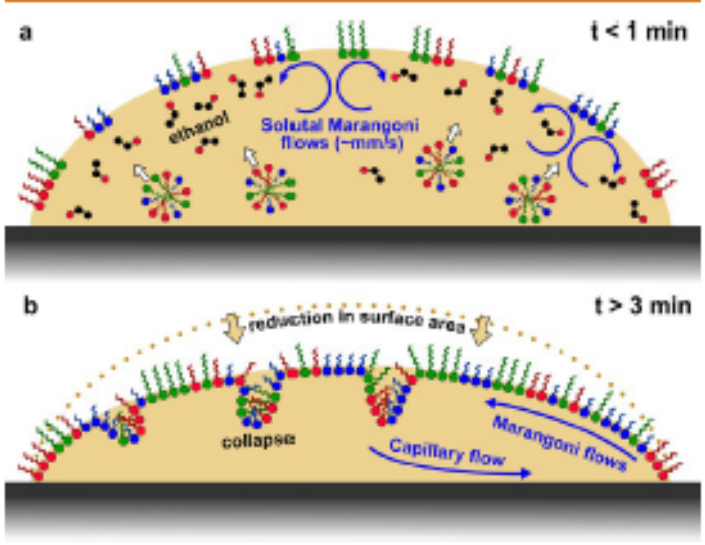

Figure 5. Schematic of the two general phases of flow and monolayer self-assembly observed during whiskey web assembly. (a) Fist phase is characterized by erratic solutal Marangoni vortices as ethanol is driven to the liquid-air interface, influencing monolayer formation and composition. (b) Monolayer collapse occurs during bulk evaporation as the interfacial area of the droplet decreases and was first observed approximately 3 min into droplet evaporation. Significantly reduced radial flows were observed.

volatile and erratic vortices ${ }^{38}$ (Figure 5a). As the concentration of ethanol is greater doser to the interface because of the lower surface tension of ethanol, ${ }^{39}$ the transport of ethanol toward the interface also drives water-insoluble chemicals toward the surface, ${ }^{26}$ where they disperse into monolayers. ${ }^{40}$ To visualize bulk fluid motion, fluorescent micropartides $(0.52 \mu \mathrm{m})$ were added to the sample and observed during evaporation of the droplet (Movie S2, Supporting Information). Vortices occur during the first phase of bulk volatile evaporation and are typical for ethanol-water droplet evaporation. ${ }^{38}$ The second phase of evaporation is characterized by a more subdued radial bulk flow, governed by thermal and surfactant-driven Marangoni stresses (Figure $5 b$ ).

Figure 6 shows the multiple length scales associated with these self-assembled structures, where square millimeter films were generated from micrometer strands of nanometer-thick monolayers The collapsed monolayers (see the scanning electron microscope (SEM) image in Figure 6 and additional SEM images in Figure S1, Supporting Information) have a strong resemblance to a traditional "twisted ribbon" fold. ${ }^{41}$

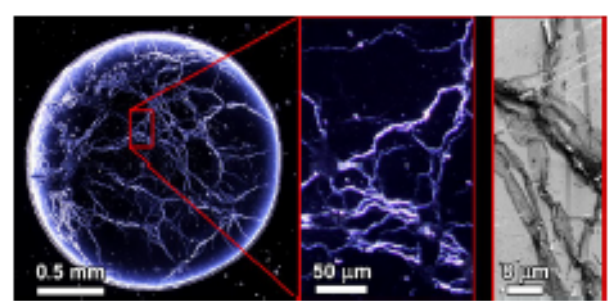

Figure 6. Magnification of the whiskey web structures SEM imaging of the whiskey web shows the distinct folds of these structures (additional SEM structures are avaibble in Figure $\$ 1$, Supporting Information).

Although folded monolayers were dearly visible using darkfield and/or light scattering techniques, confiming the presence of uncollapsed monolayers presents a greater challenge. We used energy-dispersive $\mathrm{X}$-ray spectroscopy (EDS) for elemental analysis of whiskey evaporated on TTOcoated glass slides (Figure S2, Supporting Information). There were elevated levels of carbon at the site of collapse ( $20 \%$ nomalized mass) and at uncollapsed sizes (3.5\%) relative to a plain ITO side (0.4\%), inferring that organic compounds from the whiskey were deposited on the surface of ITO and with greater concentrations at fold sites.

Intuitively, one would expect that the density of collapsed structures would increase with more hydrophobic surfaces as a larger droplet confined over smaller liquid-solid area will result in a denser material network deposit. Figure S3 (Supporting Information) does indeed demonstrate this trend when diluted whiskey droplets of larger volumes (2.0, 5.0 , and $10.0 \mu \mathrm{L}$ ) were deposited in a polytetrafluoroethylene (PTFE)-printed slide with fixed $2.0 \mathrm{~mm}$ wells. Further, droplets of less than $0.5 \mu \mathrm{L}$ did not create collapsed monolayers (data not shown). Even though the density of collapsed structures increased with larger droplet volumes, the distinct "chemical fingerprints" (Figure 1) of each brand became comparatively less distinctive and more challenging to qualitatively differentiate.

Particle image velocimetry measurements during the first phase of evaporation were conducted to explore whether or not fluid dynamics induced monolayer collapse. Previous work studying evaporation of undiluted Scotch droplets ${ }^{10}$ measured fluid vortices on the order of $100 \mu \mathrm{m} / \mathrm{s}$, whereas our diluted American whiskey samples reached velocities in excess of 10 $\mathrm{mm} / \mathrm{s}$ (Figure S4, Supporting Information). The estimated shear stress, $\tau$, associated with this flow was compared to the collapse pressure of the monolayer, evaluated as

$$
\tau \approx \mu \frac{\Delta u}{\Delta h}=\gamma / L
$$

where $\mu$ is viscosity ( $1 \mathrm{mPa} \cdot \mathrm{s}), u$ is velocity $(10 \mathrm{~mm} / \mathrm{s}), h$ is the average height of the droplet $(100 \mu \mathrm{m}), \gamma$ is the collapse pressure (on the order of $10 \mathrm{mN} / \mathrm{m}$ ), and $L$ is the length scale of the droplet $(1 \mathrm{~mm})$. The fluid shear stress was calculated to be on the order of $100 \mathrm{mPa}$, which was not enough to overcome the collapse pressure of monolayers (calculated to be $10 \mathrm{~Pa}$ for these parameters). Even though fluid shear may not directly induce monolayer collapse, it likely plays a role in the transport of compounds during heterogeneous monolayer formation or surface patterning. ${ }^{42}$ 


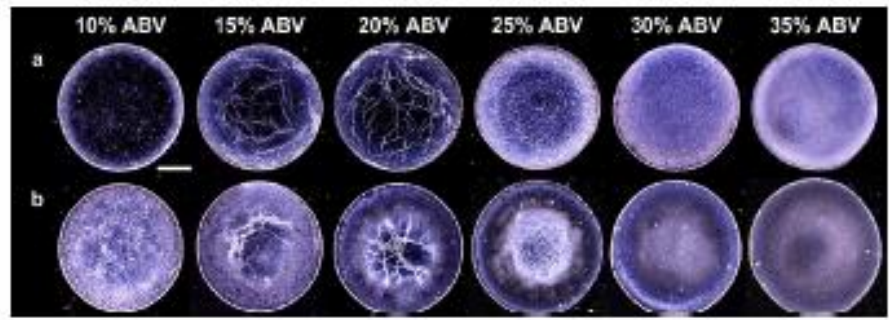

Figure 7. Effect of dilution on the deposited whiskey webs. Web formation of various aged samples: (a) 3 yeas old and (b) 23 years old. Scale bar is $0.5 \mathrm{~mm}$. Refer to Table $\$ 1$ in the Supporting Information for somple identification.
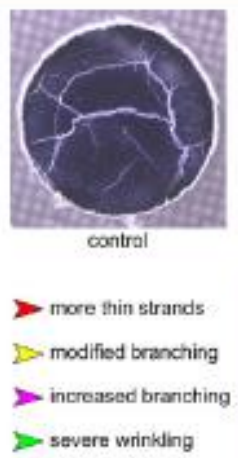

severe wrinkding
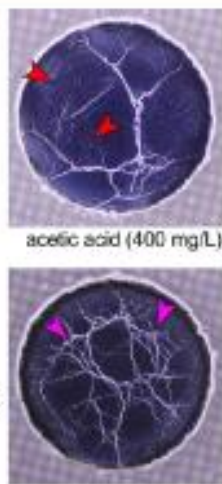

ligrin $\{400 \mathrm{mg} L$ ]

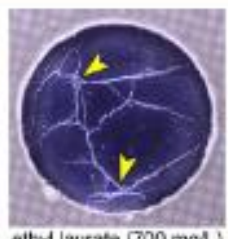

ethyl laurate $(700 \mathrm{mgh})$

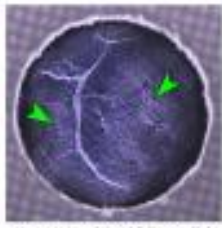

tannic acid ( $450 \mathrm{mgl}$ )
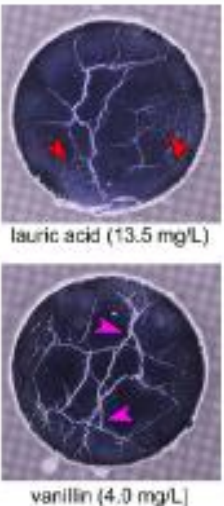

Figure 8. Effect of a set of model congenes on whiskey web patterns. Various congeners were added to a control bourbon whiskey to demonstrate that the concentration and combination of these compounds ultimately guide each whiskey web pattem.

Whiskey web formation was a function of dilution for similar sized droplets (Figure 7). In general, whiskey webs formed when various American whiskey samples were diluted within a specific range, approximately 15-25\% ABV. At alcohol concentrations lower than $10 \% \mathrm{ABV}$, most whiskey samples deposited in a simple coffee ring pattern (Figure 7a, 10\% ABV). However, longer-aged American whiskeys did not exhibit the coffee ring effect at lower alcohol concentrations (Figure $7 \mathrm{~b}, 10 \% \mathrm{ABV}$ ). Elevated levels of solutes including surfactants, occur in longer-aged samples, and the relatively high surfactant concentration may prevent the formation of a coffee ring. ${ }^{43}$ At alcohol concentrations greater than $25 \%$, the samples deposited a nearly uniform film (Figure 7, 30-35\% ABV), similarly to previous studies with Scotch ${ }^{10}$ Several factors may contribute to the absence of monolayer collapse at elevated ethanol levels First, as the ethanol concentration increases, chemical constituents become more soluble and the monolayer becomes more mobile, reducing the occurrences of collapse ${ }^{44}$ or, perhaps, alternatively facilitating the formation of vesides. Second, once the ethanol evaporates and the monolayer is established on the remaining aqueous volume, a greater ABV results in a smaller reduction in the aqueous surface area, thereby lowering the inddence of collapse. Third, previous investigations into chemical activity in the whiskey's headspace demonstrated that a higher concentration of agglomerates reduces volatility; ${ }^{45-47}$ therefore, a lower dilution (i.e., higher ABV) will reduce the magnitude and duration of the solutal Marangoni flows that impact the initialization of the monolayer itself. The data in Figure 7 show that whiskey web formation occurs for a specific combination of chemical constituents and ethanol concentration.

Oscillating pendant drop tests were conducted to measure the surface viscoelasticity of whiskey, which provides insight into the presence of surface-active molecules at the liquid-air interface. Figure S5 shows the surface tension response to sarface area oscillations for bourbon whiskey and unaged whiskey each diluted to $25 \% \mathrm{ABV}$ and $15 \% \mathrm{ABV}$. Surprisingly, data indicated that only the $15 \% \mathrm{ABV}$ bourbon whiskey sample showed measurable change in surface tension, suggesting the presence of surfactant molecules at the interface. These data can be explained by the hypotheses that bourbon whiskey sarfactants were solubilized in the bulk and/or the rigidity of the monolayer was reduced at elevated ethanol concentrations (25\% ABV) compared to reduced levels (15\% ABV). Second, these measurements show that whiskey maturation results in the generation of chemical constituents that are strongly surface-active in monolayer formation.

Thousands of chemicals are found in whiskeys, and many of them may contribute toward the molecular assembly of the collapsed monolayers described herein. The unique concentration and combination of congeners that comprise each American whiskey's flavor profile ultimately guide their correspondingly distinctive whiskey web pattems (Figure 1). To further demonstrate this, a control bourbon whiskey was spiked with one of several key constituents that have been identified in whiskeys acetic adi $(400 \mathrm{mg} / \mathrm{mL})$, ethyl laurate $(700 \mathrm{mg} / \mathrm{mL})$, lauric acid $(13.5 \mathrm{mg} / \mathrm{mL})$, lignin $(400 \mathrm{mg} /$ $\mathrm{mL})$, tannic acid $(450 \mathrm{mg} / \mathrm{mL})$, and vanillin $(4.0 \mathrm{mg} / \mathrm{mL})$. These concentrations were selected to represent the mean level 
of each respective chemical or represent that of their collective chemical group (e.g, ethyl laurate for fatty acid ethyl esters), typically found in whiskeys. ${ }^{48}$ Figure 8 shows that each congener influences the structure of a formed whiskey web in a specific way. However, predicting how every congener within whiskey will influence monolayer collapse is nontrivial. The collapse behavior of chemically homogeneous monolayers does not correlate with the response of heterogeneous monobyers. ${ }^{18}$ Also, the presence of agglomerates, particles, and impurities may result in lower than expected collapse pressures. ${ }^{22,49}$ A thorough experimental approach is likely needed to determine the influence of a particular combination of congeners in monolayer formation and collapse. For example, we demonstrated that similar structures can be created from a $1.0 \mu \mathrm{L}$ droplet of $50 \% \mathrm{ABV}$ of $1.0 \mathrm{mg} / \mathrm{mL}$ lauric acid and $1.5 \mathrm{mg} / \mathrm{mL}$ tannic acid (Figure S6, Supporting Information); the future methodical experimental manipubation of relative concentrations will provide insight into their heterogeneous performance.

Most of the tested American whiskeys (65 of 66 samples, refer to Table S1, Supporting Information) formed webs when drying $1.0 \mu \mathrm{L}$ droplets at $25 \% \mathrm{ABV}$. The distinctive visual features of the evaporated structures were generally repeatable from the same bottle of American whiskey. Even the least aged available sample, which was matured for 3 months, produced a whiskey web. However, unaged distillates at the same dilution $(n=5)$ did not form webs nor uniform films (Figure S7a, Supporting Information). The lack of web structures for unaged whiskey indicates that the components extracted during the aging process are needed to form whiskey webs.

The following aggregated image pattem identification test was conducted to evaluate the repeatability of evaporated whiskey web patterns and to demonstrate how digital image analysis could be used for identification. Three different American whiskeys from the same distiller were selected, and 30 web images were acquired for each sample ( 90 total). For a given whiskey, 25 images were analyzed and averaged to represent that sample's digital "fingerprint". Next, the remaining 15 images (five of each sample) were compared to each of the three simples' "fingerprint". The individual images were matched correctly over $90 \%$ of the time, thus demonstrating the repeatability of whiskey web formation. In the future, the identification process can be strongly enhanced by using machine learning digital algonithms for shape matching and structure identification ${ }^{5,51}$ However, it is important to note that we observed that environmental conditions (temperature, humidity, etc.) would impact web formation, and as such, their impact on monolayer integrity should be considered.

The only American whiskey sample tested that did not form webs had been aged 42 years and likely contained elevated level of surface-active compounds. The presence of the surfactant itself could disrupt the rigidity of the monolayer, resulting in a more mobile layer that is less likely to buckle. To demonstrate that elevated levels of common surfactant suppress web formation, $0.005 \mathrm{wt} \%$ of sodium dodecyl sulfate (Sigma-Aldrich, USA) was added to a control sample that otherwise formed webs - this resulted in the formation of a uniform film void of web structures.

The influence of maturation-derived surfactants on the final self-assembled pattem may also be evident in older-aged samples. This is illustrated in Figure lg,i,j,m, showing samples which were aged at least 15 years. Compared to most of the other images in Figure 1, these have reduced or nonexistent collapsed structures near the perimeter of the droplet. As the droplet evaporates, surfactants tend to have an increased concentration near the perimeter of the droplet. The relative increase in surfactant concentration at the perimeter may result in less rigid monolayers closer to the droplet's edge, thereby reducing the incidence of collapse near the perimeter.

Interestingly, webs did not form under the same conditions for diluted $1.0 \mu \mathrm{L}$ drops of non-American whiskeys such as Scotch, brandy, Irish whiskey, etc. (Figure S7b,c and Table S1, Supporting Information). These products are distilled from different mashes with various finishing processes ranging from the addition of coloring to aging in uncharred new oak barrels or used charred oak barrek. ${ }^{29}$ Recall that whiskeys derived from new charred oak barrels have greater solids content ${ }^{32}$ and relatively brger concentrations of water-insoluble content. ${ }^{31}$ Therefore, the reduced overall concentration of solutes, induding water-insoluble components, is likely why monolayer collapse was not observed under similar conditions (20-25\% ABV, $1.0 \mu \mathrm{L}$ ). However, we did find that two non-American whiskeys produced self-assembled structures with a greater droplet volume $(2.0 \mu \mathrm{L})$ and lower dilution (40\% ABV); refer to Figure S8, Supporting Information.

\section{CONCLUSIONS}

In summary, the results reported here show that whiskeys are chemically complex liquids whose chemical profiles not onty distinguish each product ${ }^{52}$ but are also responsible for the uniqueness of each product-specific pattern of deposits from drying droplets. Our findings strongly suggest that the new charred barrels have components that readily dissolve in ethanol, which form colloidal suspensions when diluted with water due to nanopartide precipitation or solubilization (though this is not the only alcoholic spint that forms colloids with water dilution). ${ }^{53}$ These constituents derived from new barrel maturation are critical to whiskey web formation. Further, it may be possible to form these structures using samples other than American whiskeys under conditions that exacerbate web formation induding solutions that encompass greater levels of similar colloids and/or evaporation on more hydrophobic surfaces (i.e., greater change in surface area during evaporation),

One unexpected and visually impressive finding is that congeners, comprising less than 1\% (by weight) of whiskey composition, are responsible for the creation of self-assembled structures that span several length scales, beginning with the collapse of sub-micrometer monolayers from nanoscale agglomerates to the final weblike structure that covers several square millimeters. Use of digital image analysis may enable visual identification of the product and degree of (desired or not) dilution. For example, web pattems become less dense with increasing dilution (Figure 7, 15-25\% ABV), and a straightforward digital image analysis tool can be used to identify adulterated whiskey. To this end, we assembled a simple smartphone-based visualization platform (Figure S9, Supporting Information), enabling portable acquistion of whiskey web digital images. Our preliminary data show that sach digital image inspection may lead to simple chemical analysis of American whiskeys; furthermore, this simple and surprisingly distinctive effect may be applicable to similar characterization of spirits ${ }^{54}$ or other volatile liquids. 


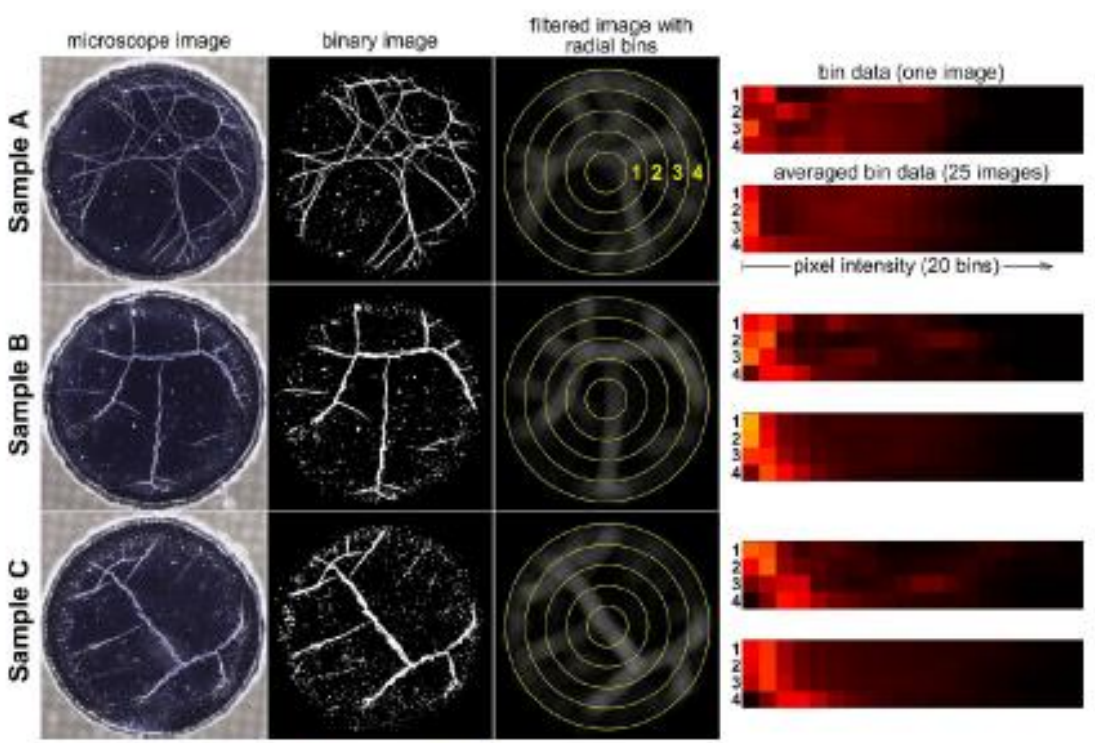

Figure 9. Analysis of microscope inages of web pattems Thirty droplets of each tested American whiskey sample were imaged and analyzed. Digital images were converted to binary images and processed with a radial filter. Each pixel was then placed into a bin based on their radial position (four bins) and intensity ( 20 bins). Twenty-five anrays were averaged to represent a given American whiskey sample. The remaining 15 droplets (five of each sample) were tested aga inst the averaged represent ative data. A single image was matched with its American whiskey over $90 \%$ of the time. Refer to Table $\$ 1$ in the Supporting Information for sample identification.

\section{METHODS}

Three different substrates wete ised for the evaporation of droplets: PTFE-printed slides containing $302.0 \mathrm{~mm}$ wells (Electron Microscopy Science, \#63434-02, Hatfield, PA), microscope cover glass $(22 \times 50 \mathrm{~mm}, \mathrm{VWR}, \# 16004314$, Radnor, PA), and indium tin ovide (ITO) glass slides (SPI Supplies, West Chester, PA). The hatter were used exchusively for SEM images and EDS analysis Substrates were submerged in a sonicated acetone bath for at least five minutes prior to testing

Whiskey samples (Table S1, Supporting Information) were either acquired through commercial purchase, generously donated by colleagues, or provided by the distiller. Whiskey samples were diluted with deionized water and mixed prior to droplet deposition. Evaporation occurred under ambient conditions $\left(20-22^{\circ} \mathrm{C}, 30-\right.$ $46 \% \mathrm{RH})$.

Microscopic images were captured using a digital camera (Canon EOS Rebel T7i) mounted to an inverted microscope (Nikon Ti-U) or an upright microscope (Zeiss Avio Imager). For the inverted microscope, an LED ring light was mounted above the sample and its height was adjusted for a given microscope objective $(2 x, 4 \times, 10 x$, or 20x) for optimal image contrast. A dark field phase contrast ring was used in conjunction with the upright microscope. The dighal images were not altered except for minor adjustments in contrast levels.

For EDS measurements, Bruker QUANTAX XFlash 6 energy. dispersive $X$ ray spectroscopy was used with Garl Zeiss SUPRA 35 VP SEM (Bruker Nano GmBH, Berlin, Germany, Carl Zeiss Microscopy, GmBH, on745 Jena, Gemany).

Fluorescent microparticles $(1.0 \mu \mathrm{m}$, Themo Scientific Fluoro-max R0100) were added to the diluted whiskey semples for microparticle image velocimetry (simibr to that in ref 10), with a final concentration of $0.01 \%$ solids A $25 \%$ ABV $1.0 \mu \mathrm{L}$ droplet sample was deposited on a cle aned PTFE-printed slide Image pairs $(\Delta t=1.4$ ms) were acquired every $0.24 \mathrm{~s}$ with a highspeed camera (HiSpec 4, Fastec Imaging) mounted to an inverted microscope (Nikon Ti-U, $4 \times$ objective with $0.13 \mathrm{NA}$ ). Particles were iluminated with scattered light using an LED ring light
Interfucial rheology was conducted via the pendant drop technique using a Ramé-Hart goniometer. Bourbon whiskey and unaged whiskey were diluted with deionized water to 25 and $15 \%$ ABV. Liquid droplets of approximately $8 \mu \mathrm{L}$. were dispensed into a sealed cuvette and surrounded by air. The droplets were then left to rest for up to 30 min. As the droplets aged, evaporation reduced the droplet wolume To compensate for this, small volumes of the liquid were dispensed periodically to maintain a volume of $8 \mu \mathrm{L}$. Oscillations were conducted at a frequency of $0.1 \mathrm{~Hz}$ and an area displacement $(\Delta \mathrm{A} /$ $\left.A_{0}\right)$ of less than $10 \%$ to ensure a mechanical equilibrium between the droplet and the oscillator. Each droplet was oscillated five times to obtain the corresponding change in surface tension. After the oscillation was complete, the droplet was discarded, and a new droplet was formed. This process was repeated in triplicate for each sample, and the results were reported as an average of the three runs. Congener tests were conducted with acetic acid (Sigma-Aldrich, \#A6283), ethyl laurate (Sigma-Aldrich, \#W244104), lauric acid (Sigma-Aldrich, \#W261408), lignin ( $\mathrm{TCl}$ America, CAS 8068.05 1), tannic acid (Sigmo-Aldrich, \#403040), and vanillin (SigmaAldrich, $¥ \mathrm{~V} 1104)$. Congeners were added to a control bourbon whiskey first and mixed before being diluted with deionized water. Two microliter droplets were applied to PTFE printed slides and evaporated (Figure 4); similar qualitative results were observed for 10 $\mu \mathrm{L}$. droplets, but such features were exacerbated using the larger droplet volume.

Three different bourbon whiskeys from the same distiller were selected for repeatability and identification tests (Figure 9). Simples were first diluted to $25 \% \mathrm{ABV}$, and then $301.0 \mu \mathrm{L}$ drops of each simple ( 90 drops total) were evaporated on PTFE printed slides. Digital images were acquired for each image with an inverted microscope as previously described. A custom MATLAB program was developed for the following analytical procedure. First, digital images (2.4 MP) were converted to a binary inuge using a threshold function (where black pixels have an intensity value of " $\sigma^{\prime \prime}$ and white pixels ${ }^{\prime \prime} 1^{n}$ ). Next, a circle (of radius $R$ ) was fit to each well, and only pirels with in this circle were subsequently analyzed (approximately 7.2 million pixels). Nent, a circular averaging filter (with filter radius $0.1 R$ ) was applied to each binary image to effectively blur the image. The 
resulting pirels were then placed into data bins by radius (five bins at equal $0.2 R$ increments) and intensity (20 bins at equal increments across a range of 0 to 0.4$)$. Numerical values of each bin were the percentage of pirels within a certain radial bin found within a specific intensity bin. The first radial bin $(0$ to $0.2 R)$ was omitted, resulting in a final $4 \times 20$ data array to represent a single digital web imuge. A data array was obtained for each of the 90 web images A second MATLAB program was created in which, for a given whiskey sample of 30 image data anrays, 25 were randomly selected and averaged as a representation of that whiskey sample. The remaining 15 samples (five for each of the three smples) were compared to the three somples' averaged data array using a sum of least squares; the value with the smallest sum was selected as its match. This program was repeated 1000 times and matched individual images successully over $90 \%$ of the time.

Smartphone images were acquired with a clip-on lens (Kingmas $60 \mathrm{X}$ ) mounted on a Simsung Galury Note 8 smartphone.

\section{ASSOCIATED CONTENT}

\section{- Supporting Information}

The Supporting Information is available free of charge at https://pubs.acs.org/doi/10.1021/acsnano.9b08984.

SEM images (Figure S1), EDS analysis (Figure S2), results from a web density study (Figure S3), partide image velocimetry results (Figure S4), oscillating pendant drop results (Figure S5), image of a synthetic weblike pattern (Figure S6), non-American whiskey patterns (Figures S7 and S8), and smartphone visualization (Figure S9) (PDF)

Movie S1 (MP4)

Movie S2 (MP4)

\section{AUTHOR INFORMATION}

\section{Corresponding Author}

Stuart J. Williams - Department of Mecharical Engineering

University of Louisville, Lotisville, Kentucky 40292, Unied

States; ordidorg/0000-0002-1678-7544;

Email: stuartwilliams@olouisville.edu

\section{Authors}

Adam D. Carrithers - Department of Mechaniaal Engineering, University of Lotisville, Lotisville, Kentucky 40292, Uniked States

Martin J. Brown, VI - Dequrtment of Mecharical Engineaing, University of Louisville, Lotisville, Kentucky 40292, United States

Mohamed Z. Rashed - Department of Mechanical Engineering, University of Lotisville, Lotisville, Kentucky 40292, United States

Sabina Isham - Department of Chemical and Biomolecular Engineering, North Carolina State University, Rakigh, North Carolina 27606, United States

Orlin D. Velev - Deparbment of Chemical and Biomolecular Engineering, North Carolina State University, Rakigh, North Carolina 27606, United State; (1) orcid_org/0000-00030473-8056

Complete contact information is available at:

https://pubs.acs.org/10.1021/acsnano.9b08984

\section{Author Contributions}

${ }^{5}$ M.J.B. and A.D.C. contributed equally to this work. Notes

The authors declare no competing finandial interest.

\section{ACKNOWLEDGMENTS}

The authors would like to adknowledge those who generously donated samples for this study induding E. Downs (Limestone Branch Distillery), T. Effler (Brown-Forman Corp.), P. Heist (Wildemess Trail Distillery), S. Herman (Louisville Metro), J. Kepley (Adaptive Nursing \& Healthcare Services), D. Mandell (Bardstown Bourbon Company), C. Miller (Kentucky Artisan Distillery), G. Miller (University of Califomia, Davis), C. Morris (Woodford Reserve Distillery), M. Niemann (Four Roses Distillery), L. Tompkins (University of Louisville), P. Van Winkle (Old Rip Van Winkle Distillery), A.H. Williams (brother of S.J. Williams), C. Zaborowski (Westport Whiskey \& Wine), and K. Zamanian (Rabbit Hole Distillery). The authors greatly apprediate K.A. Erk and C.R. Davis (Purdue University) for performing the pendant drop tests. The authors appredate valuable scientific conversations with $T$. Collins (Washington State University) and W. Ristenpart (University of Califomia, Davis).

\section{REFERENCES}

(1) Poulard, C,; Damman, P. Control of Spreading and Drying of a Polymer Solution from Marangoni Flows. Europhys. Lett. 2007, \$0, 64001

(2) Sefiane, K.; Tadrist, L; Douglas, M. Experimental Study of Evaporating Water-Ethanol Mirture Sessile Drop: Influence of Concentration. Inf. J. Heat Mass Transfer 2003, 46, 4527-4534.

(3) Yunker, P. J.; Still, T.; Lohr, M. A.; Yodh, A. G. Suppression of the Coffee-Ring Fffect by Shape-Dependent Capillary Interactions Nafure 2011, 476, 306-311.

(4) Anyfintalis, M.; Geng, Z; Morel, M; Rudiul, S.; Baigl, D. Modulation of the Coffee-Ring Effect in Particle/Surfactant Mixtures: The Importance of Particle-Interface Interactions Langmaer 2015, 31 , 4113-4120.

(5) Girard, F.; Antoni, M; Faure, S; Steinchen, A. Influence of Heating Temperature and Relative Humidity in the Evaporation of Pinned Droplets. Colloids Surf, A 2008, 323, 36-49.

(6) Girard, F.; Antoni, M Influence of Substrate Heating on the Evaporation Dynamics of Pinned Water Droplets Langmaer 2008, 24, $11342-11345$

(7) Kuang, M.; Wang, L.; Song Y. Controllable Printing Droplets for High-Resolution Pattems. Adv. Mater. 2014, 26, 6950-6958.

(8) Deegan, R. D.; Bakajin, O,; Dupont, T. F.; Huber, G.; Nagel, S.

R; Witten, T. A Capillary Flow as the Cause of Ring Stains from Dried Liquid Drops. Nafure 1997, 389, 827.

(9) Mampallil, D.; Eral, H. B. A Review on Suppression and Utilization of the Coffee-Ring Effect. Adv. Colbid Interface Sci 2018, $252,38-54$.

(10) Kim, H.; Boulogne, F.; Um, E; Jacobi, L; Button, E.; Stone, H. A. Controlled Uniform Coating from the Interplay of Marangoni Flows and Surface-Adsorbed Macromolecules. Phys Rev. Lett 2016, $116,124501$.

(11) Williams, S. J-; Brown, M. J.; Garrithers, A. D. Whiskey Webs: Microscale "Fingerprints" of Bourbon Whiskey. Phys. Rev. Fhids $2019,4,100511$

(12) Mohwald, H. Phospholipid and Phospholipid Protein Monohyers at the Air/Water Interfice. Avvu. Rev. Phys. Chom. 1990, 41, $441-476$.

(13) Zasadrinski, J. A; Viswanathan, R; Madsen, L_; Gamaes, J-; Schwart, D. K. Langmuir-Blodgett-Films. Science 1994, 263, 17261733.

(14) Phan, M. D.; Lee, J-; Shin, K. Collapsed States of Langmuir Monolayers J. Oleo Sii 2016, 65, 385-397.

(15) Baldelli, S.; Schnitzer, C.; Simonelli, D. Aqueous Solition/Air Interfaces Probed with Sum Frequency Generation Spectroscopy. J. Phys. Chem. B 2002, 106, 5313-5324. 
(16) Lin, J-; Conboy, J. C. Phase Transition of a Single Lipid Bilayer Measured by Sum-Frequency Vibrational Spectroscopy. J. Am. Chem. Sac 2004, 126, 8894-8895.

(17) McConnell, H. M. Structures and Transitions in Lipid Monolayers at the Air-Water-Interfice. Annu. Rev. Phys Chem. $1991,42,171-195$.

(18) Carter-Fenk, K. A.; Allen, H. C. Collapse Mechanisms of Nascent and Aged Sea Spray Aerosol Prory Films. Atmosphere 2018, 9. 503.

(19) Alonso, C.; Alig T.; Yoon, J-; Bringezu, F.; Warriner, $\mathrm{H}_{\text {.; }}$ Zasadrainshi, J. A. More Than a Monolayer: Relating Lung Surfactant Structure and Mechanics to Composition. Biophys J. 2004, 87,41884202

(20) Gopal, A, Lee, K. Y. C. Morphology and Collapse Transitions in Binary Phospholipid Monolayers. $J$. Phys. Chom B 2001, 105 , $10348-10354$.

(21) Angelova, A.; Vollhardt, D.; Ionov, R. 2D-3D Transformations of Amphiphilic Monolayers Influenced by Intermolecular Interac tions: A Brewster Angle Microscopy Study. J. Phys. Chem. 1996, 100, $10710-10720$.

(22) Ybert, C.; La, W.; Moller, G.; Knobler, C. M Kinetics of Phase Transitions in Monolayers: Collapse. J. Phys: Condors Maft $\sigma$ 2002, $14,4753-4762$

(23) Lee, K. Y. C. Cdlapse Mechanisms of Langmuir Monolayers Annu. Rev. Phys Chom 2008, 59, 771-791.

(24) Lipp, M. M.; Lee, K. Y. C.; Tala moto, D. Y.; Zasadrinshi, J. A. Waring A. J. Coeristence of Buckled and Flat Monolayers. Phys. Rev. Lett. 1998, 81, 1650-1653.

(25) Kelly, A. G.; Vega-Mayoral, V.; Boland, J. B.; Coleman, J. N. Whiskey-Phase Evfoliation: Eufoliation and Printing of Nanosheets Using Irish Whiskey. 2D Mata. 2019, 6, 045036.

(26) Karkson, B. C. G.; Friedman, R. Dilution of Whisky - The Molecular Perspective. Si. Rep. 2017, 7, 6489.

(27) Russell, I. Whisky: Tednology, Production and Marketing: Academic Press: Boston, MA, 2003.

(28) Piggott, J. R.; Sharp, R, Duncan, R. E. B. The Saince and Tednology of Whislies; John Wiley \& Sons: New York, 1989.

(29) Bujake, J. E. Beverage Spirits, Distilled. Kirk-Othmer Encydopedia of Chanical Teclorology; John Wiley \& Sons: New York, 2007 .

(30) Liebmann, A. J-; Rosenblatt, M. Changes in Whisky While Maturing. Ind. Eng. Chon. 1943, 35, 994-1002.

(31) Crampton, C. A.; Tolman, L. M. A Study of the Changes Taling Place in Whiskey Stored in Wood. J. Am. Chom. Sac 1908, 30 , $98-136$.

(32) Beverage Spirits, Distilled. In Kirk-Ofimer Encydopelia of Chomical Tedinology, 3rd ed.; John Wiley and Sons: New York, 1978; Vol. 3, p 837 .

(33) Laidler, K. J-; Meiser, J. H. Physical Chanistry; Benjamin/ Cummings Pub. Co.: Menlo Park, CA, 1980.

(34) Birdi, K S. Seff-Assonbly Monolayo Structures of Lipids and Macomoleales at Interface; Kluwer Academic/Plenum Publishers: New York, 1999.

(35) Cheng, A. K. H.; Soolaman, D. M.; Yu, H.-Z. Non-Evaporating" Microdroplets on Self Assembled Monolayer Surfaces under Ambient Conditions $J$. Phys Chon. B 2007, 111, 7561-7566.

(36) Man sfield, W. W.; Mysels, K. J.; Wu, J. Evaporation Retardation by Monolayers Science 1972, 176, 944-945.

(37) Bennacer, R; Sefiane, K. Vortices, Dissipation and Flow Transition in Volatile Binary Drops. J. Fhuid Mech. 2014, 749, 649665.

(38) Christy, J. R. E.; Hamamoto, Y.; Sefiane, K. Flow Transition within an Evaporating Binary Minture Sessile Drop. Phys. Rev. Latt. 2011, 106, 205701.

(39) Liu, C ; Bonaccurso, E.; Butt, H.J. Evaporation of Sessile Water/Ethanol Drops in a Controlled Environment. Phys. Chem. Chan. Phys 2008, 10, 7150-7157.
(40) Ries, H. E. Heart-Shaped Monolayer Islands and Ridged Collapse Structures of Ceramide Galactoside. J. Colloid Interface $\mathrm{Sa}$ $1982,88,298-301$.

(41) Ries, H. E; Swiff, H. Twisted Double Layer Ribbons and the Mechanism for Monolayer Collapse. Langmuir 1987, 3, 853-855.

(42) Bassou, N-; Rharbi, Y. Role of Benard-Marangoni Instabilities during Solvent Evaporation in Polymer Surface Corrugations Langmuir 2009, 25, 624-32.

(43) $\mathrm{Hu}, \mathrm{H}$.; Larson, R. G. Analysis of the Effects of Marangoni Stresses on the Microflow in an Evaporating Sessile Droplet. Langmair $2005,21,3972-3980$.

(44) Bhamla, M. S.; Chai, C.; Alvarez-Valenzueh, M. A; Tajuelo, J-; Fuller, G. G. Interficial Mechanisms for Stability of Surfactant-Laden Films. PloS One 2017, 12, e0175753.

(45) Pigott, J. R.; Gonzalez Vinas, M. A; Conner, J. M.; Withers, S. J-; Paterson, A. Effect of Chill Filtration on Whisky Composition and Headspace. In Flavow Saience: Recent Developmorts; Taylor, A. J. Mottram, D. S., Eds; Woodhead Publisher: Cambridge, U.K., 1997.

(46) Conner, J. M; Piggott, J. R.; Paterson, A; Withers, S Interactions between Wood and Distillate Components in Matured Scotch Whisky. In Favow Saience: Recent Developmonts; Taylor, A. J, Mottram, D. S., Eds; Woodhead Publisher: Cambridge, U.K., 1997.

(47) Boothroyd, E. L.; Linforth, R. S. T.; Cook, D. J. Effects of Ethanol and Long-Chain Ethyl Ester Concentrations on Volatile Partitioning in a Whisky Model System. J. Agric Food Chan. 2012, 60, $9959-9966$.

(48) Freitas, R. A. Whiskzy Machine: Nanofactary-Based Replication of Fine Spinits and Other A lcohol-Rased Bevoages; http://wwwimm_org/ Reports/rep047.pdf (accessed 2020-02-26).

(49) Kuo, C.C.; Kodama, A. T,; Boatwright, T,; Dennin, M. Particle Size Effects on Collapse in Monolayers. Langmair 2012, 28, 1397613983.

(50) Reinhart, W. F-j Long, A. W.; Howard, M. P.; Ferguson, A. L_; Panagiotopoulos, A. Z. Machine Learming for Autonomous Crystal Structure Identification. Soft Matter 2017, 13, 4733-4745.

(51) Phillips, C. L; Voth, G. A. Discovering Crystals Using Shape Matching and Machine Learning. Soft Matter 2013, 9, 8552-8568.

(52) Collins, T. S.; Zweigenbaum, J-; Ebeler, S. E. Profiling of Nonvolatiles in Whiskeys Using Ultra High Pressure Liquid Chromatography Quadrupole Time-ofFlight Mass Spectrometry (UHPLC QTOF MS). Food Chan. 2014, 163, 186-196.

(53) Vitale, S. A.; Katz, J. L. Liquid Droplet Dispersions Formed by Homogeneous Liquid-Liquid Nucleation: "The Ouzo Effect. Langmair 2003, 19, 4105-4110.

(54) Gonzalez-Gutierrez, J-; Perez-Lsidoro, R,; Ruiz-Suarez, J. C. A Technique Based on Droplet Evaporation to Recognize Alcoholic Drinks. Rev. Sai Instion. 2017, \&8, 074101. 
Supporting Information

Multiscale Self-Assembly of Distinctive Web-Like Structures from Evaporated Drops of Dilute American Whiskeys

Authors:

Adam D. Carrithers ${ }^{1 \dagger}$, Martin J. Brown VI ${ }^{1 \dagger}$, Mohamed Z. Rashed ${ }^{1}$, Sabina Islam ${ }^{2}$, Orlin D. Velev $^{2}$, Stuart J. Williams ${ }^{1 *}$

Affiliations:

${ }^{1}$ Department of Mechanical Engineering, University of Louisville, Louisville, KY, 40292, USA.

${ }^{2}$ Department of Chemical and Biomolecular Engineering, North Carolina State University,

Raleigh, NC, 27606, USA.

*Correspondence to: stuart.williams@louisville.edu.

$\dagger$ Authors had equal contributions. 


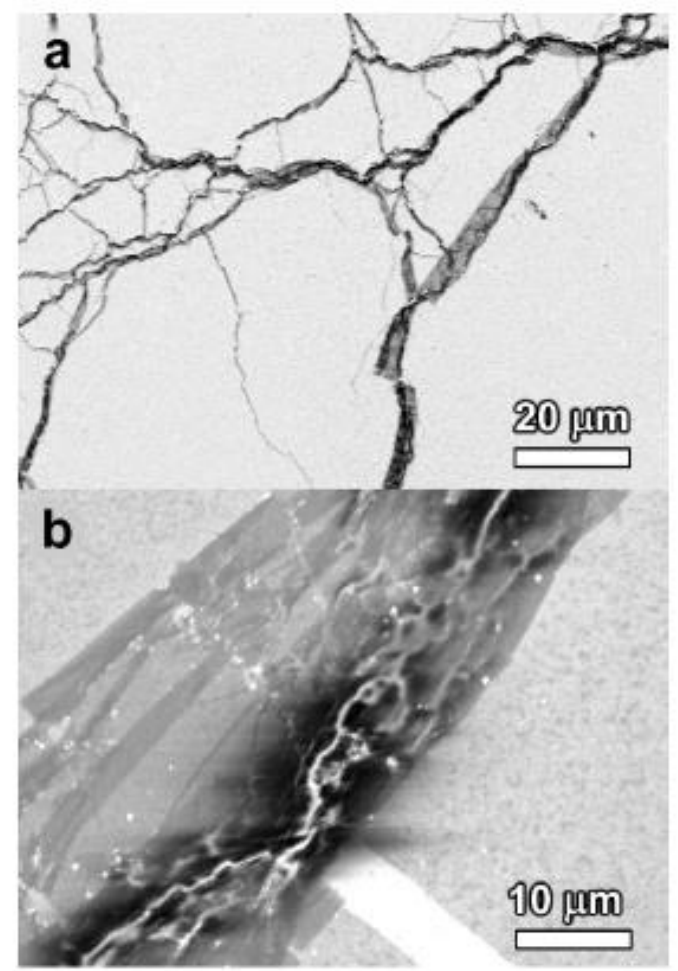

Figure S1. SEM images of whiskey webs. High-resolution SEM images of structures formed by two different American whiskeys. Refer to Table S1 for sample identification. 

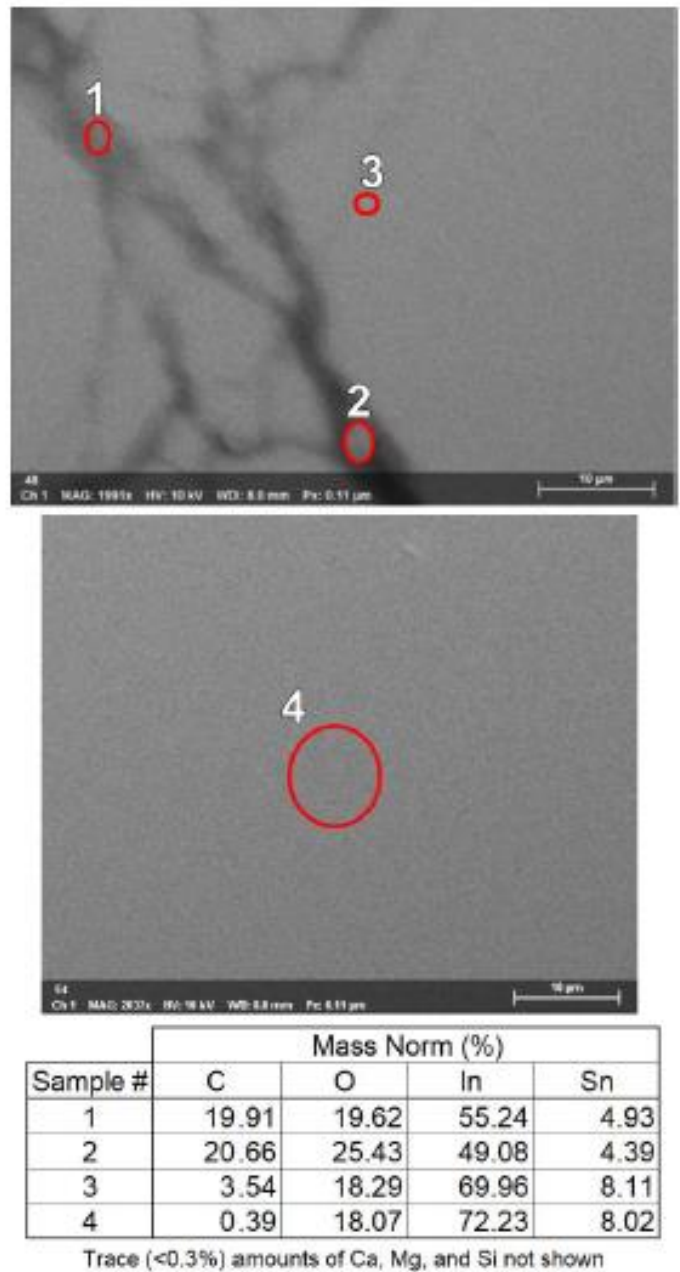

Figure S2. EDS analysis of whiskey web structures assembled on an ITO-coated glass slide. Points (1) and (2) show elevated levels of carbon compared to (4) plain ITO. Point (3) measured an area that did not experience collapse and its carbon levels are also elevated compared to (4), suggesting the presence of a monolayer or other chemicals originating from the whiskey. 

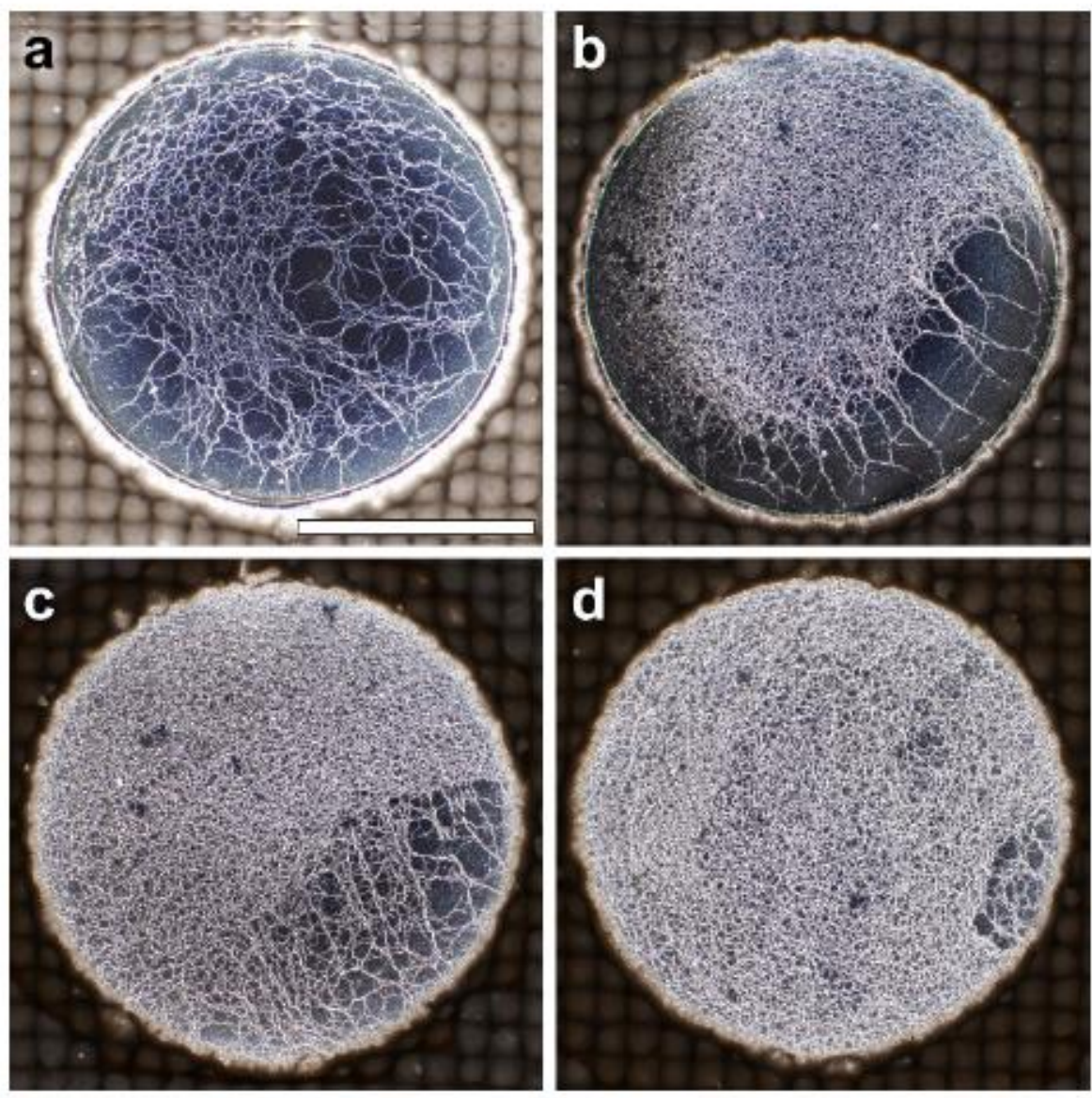

Figure S3. Monolayer folding density as a function of diluted ( $25 \% \mathrm{ABV})$ droplet volume deposited on a printed PTFE slide with $2.0 \mathrm{~mm}$ wells. Web density increased as the droplet volume increased with (a) $1.0 \mu \mathrm{L}$, (b) $2.0 \mu \mathrm{L}$, (3) $5.0 \mu \mathrm{L}$, and (d) $10.0 \mu \mathrm{L}$. Scale bar is $1.0 \mathrm{~mm}$. This whiskey was a 48 month old barrel-proof sample (originally $65 \% \mathrm{ABV}$ ) provided by Brown-Forman Corporation. 

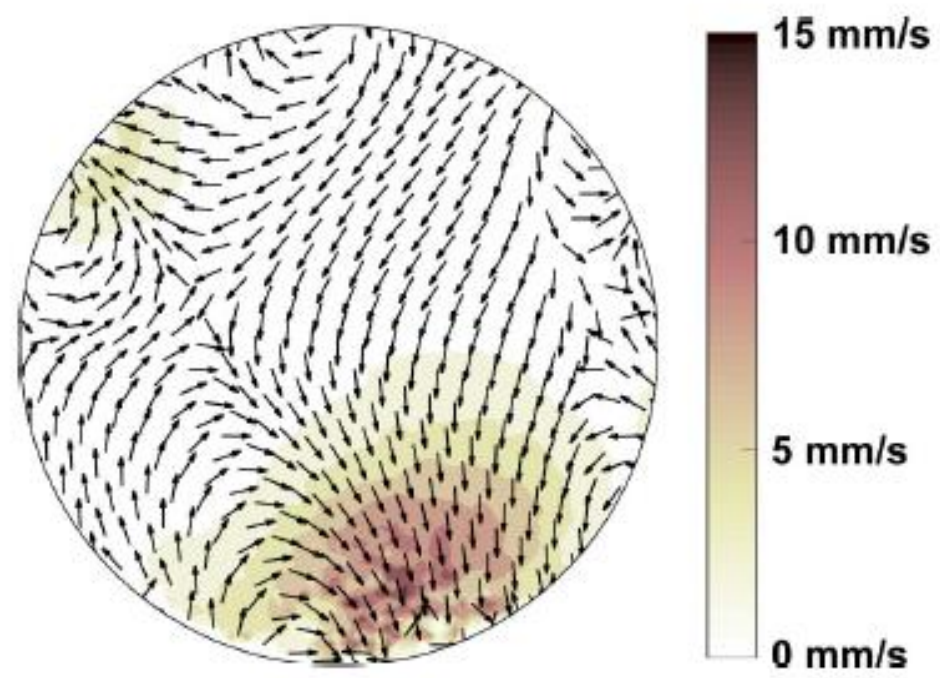

Figure S4. A representative frame of particle image velocimetry measurements during the first phase of droplet evaporation. This demonstrates the relatively high velocities $(\sim 10 \mathrm{~mm} / \mathrm{s})$ observed during this phase of evaporation. 

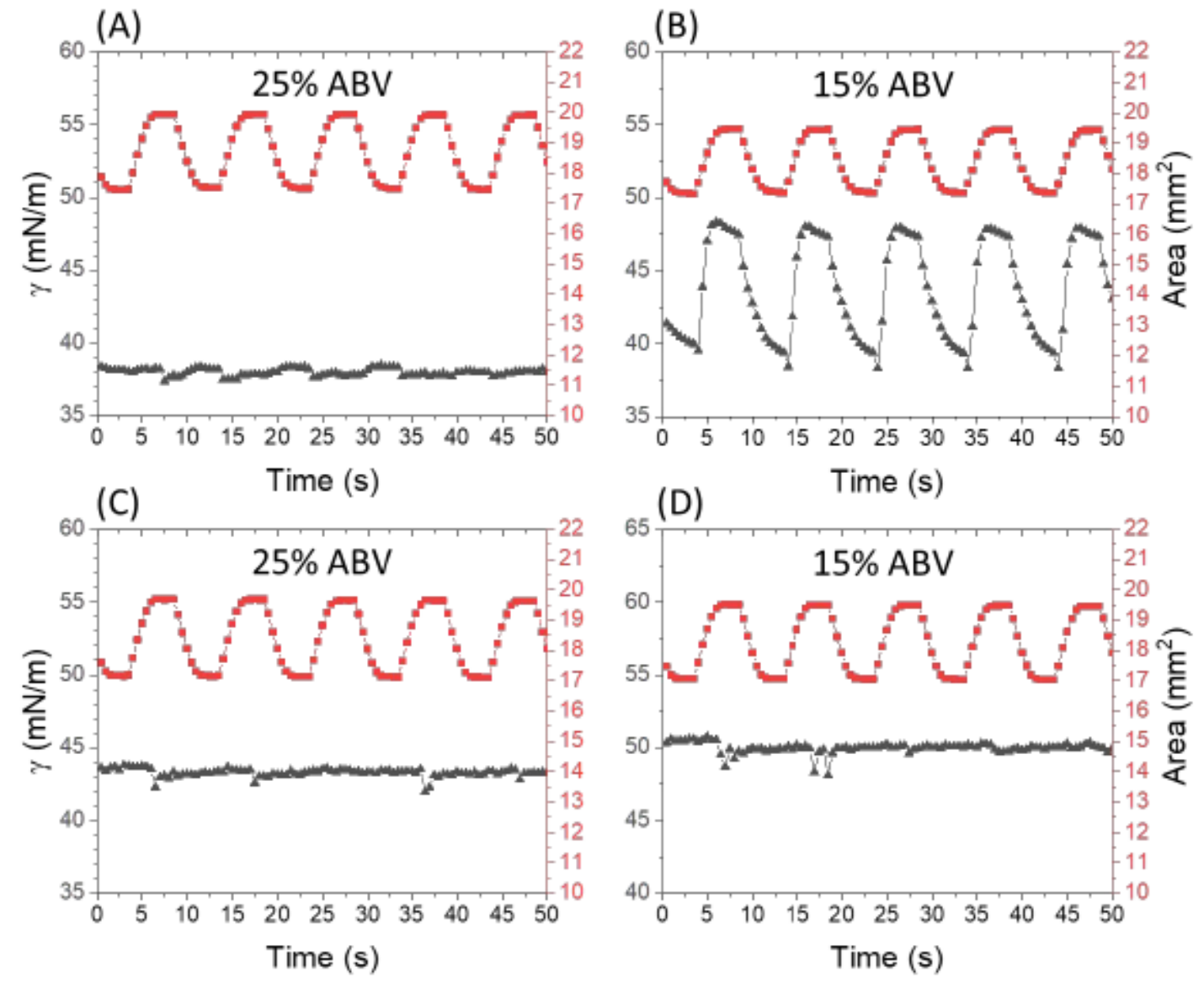

Figure S5. Results from pendent drop technique to determine surface viscoelasticity for (A) bourbon whiskey at $25 \% \mathrm{ABV}$, (B) bourbon whiskey at $15 \% \mathrm{ABV}$, (C) unaged whiskey at $25 \%$ $\mathrm{ABV}$, and (D) unaged whiskey at $15 \% \mathrm{ABV}$. The measured surface tension (grey triangles) in response to surface area oscillation (red squares) shows that the $15 \% \mathrm{ABV}$ bourbon whiskey has a response consistent with a surfactant-laden surface. Refer to Table S1 for sample identification. 


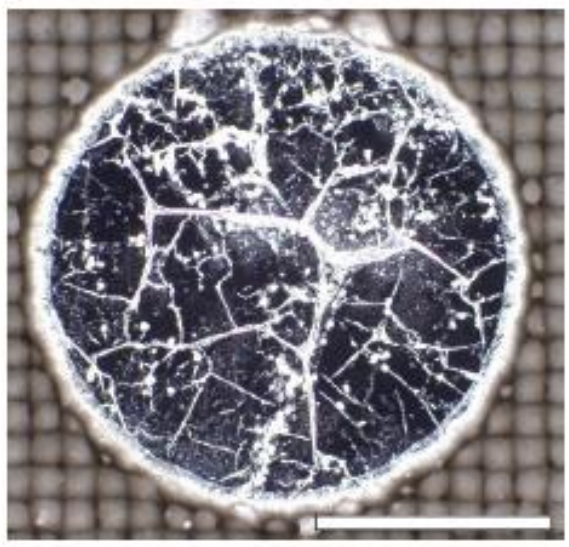

Figure S6. The resulting folded structure using a $1.0 \mu \mathrm{L}$ droplet of $50 \% \mathrm{ABV}$ of $1.0 \mathrm{mg} / \mathrm{mL}$ lauric acid and $1.5 \mathrm{mg} / \mathrm{mL}$ tannic acid deposited on a PTFE printed slide. Scale bar is $1.0 \mathrm{~mm}$.

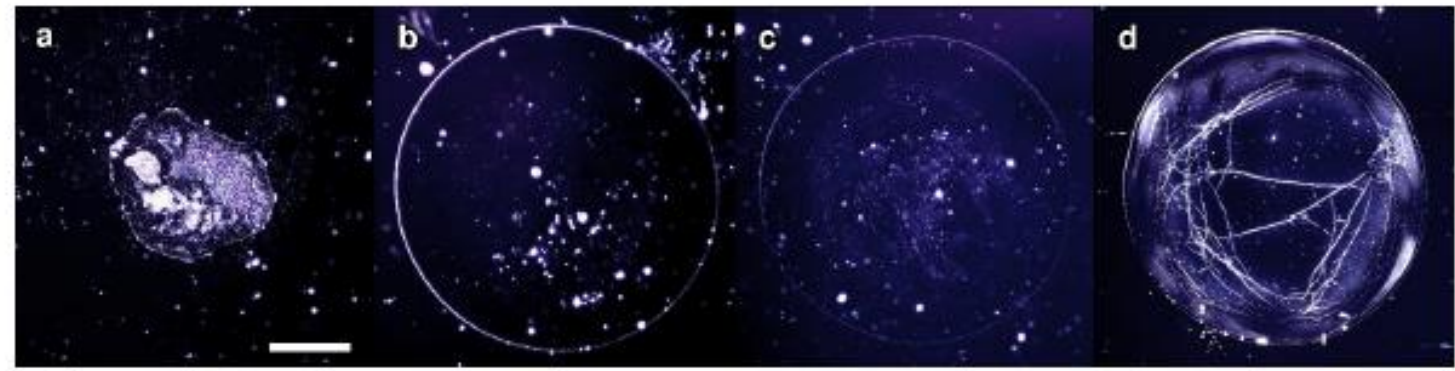

Figure S7. Whiskey webs were unique to American whiskey. Web features did not form for $20 \%$ ABV $1.0 \mu \mathrm{L}$ droplets for tested (a) unaged whiskeys nor (b, c) non-American whiskeys. (d) An American whiskey on the same glass substrate. Scale bar is $0.5 \mathrm{~mm}$. Refer to Table S1 for sample identification. 


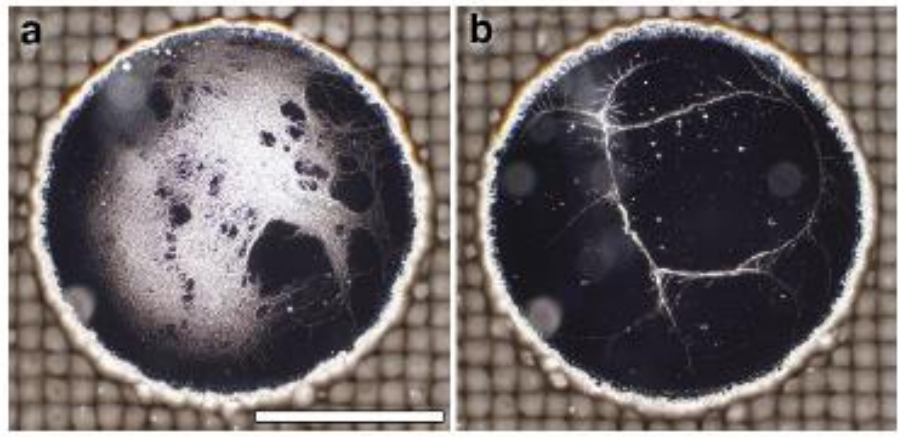

Figure S8. Two non-American whiskeys formed structures at a greater droplet volume $(2.0 \mu \mathrm{L})$ and lower dilution ( $40 \% \mathrm{ABV}$ ) when deposited on a PTFE slide. Scale bar is $1.0 \mathrm{~mm}$. Refer to Table S1 for sample identification.
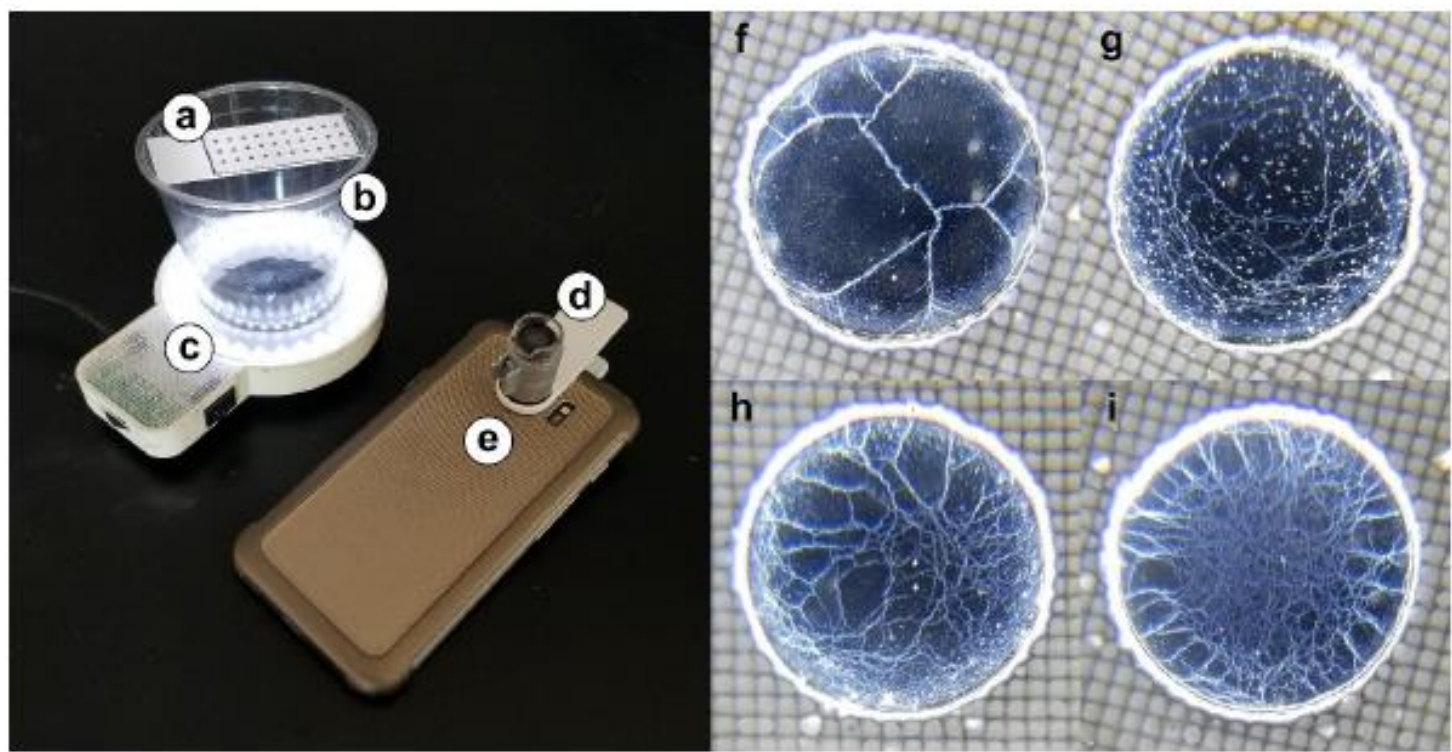

Figure S9. Smartphone visualization of whiskey webs. Rudimentary visualization system where whiskey webs can be visualized using (a) a PTFE printed slide, (b) an elevated slide holder, (c) a LED ring light, and a (d) a clip-on microscope lens for (e) a smartphone. Four different samples

(f-i) were imaged using this setup. Refer to Table S1, Supporting Information for sample identification. 
Table Sl. A list of the samples tested at $25 \%$ ABV $1.0 \mu \mathrm{L}$ on a printed PTFE slide containing

\section{$2.0 \mathrm{~mm}$ wells.}

\begin{tabular}{|c|c|c|c|c|c|}
\hline Sample Name Untelb Flarurel & $\begin{array}{l}\text { Distillary or } \\
\text { Manufacturer }\end{array}$ & $\begin{array}{l}\text { Onig } \\
\text { ABV }\end{array}$ & Sample Name CArtblo Flourol & $\begin{array}{l}\text { Distiliery or } \\
\text { Manufacturer }\end{array}$ & $\begin{array}{l}\text { Orig } \\
\text { ABV }\end{array}$ \\
\hline 1792 Port Finish & Barton 1792 & 44.5 & Van Winkle Special Reserve 12 Year [1d] & Buftailo Trace & 45.2 \\
\hline 1792 Small Batch [1k] & Barton 1792 & 46.9 & W.L. Weller 12 Year [9, Samplo C] & Buftalo Trace & 45.0 \\
\hline Beer Barrel Bourbon & New Holand & 40.0 & Whiskey ROW [s9h] & Kentucky Artisan & 44.0 \\
\hline Blanton's & Buffalo Trace & 63.4 & Wild Turkey Rare Breed & Wild Turkey & 56.4 \\
\hline Booker's & $\mathrm{Jm}$ Beam & 63.5 & Wildemess Tral & Wildemess Tral & 50.0 \\
\hline Bulleit Bourion & Disageo & 45.0 & Woodford Reserve, Double Oaked [tf, s1b] & Brown-Forman & 45.2 \\
\hline Cleveland Whiskey Black Reserve & Cleveland Whiskey & 50.0 & Yellowstione Limted Edlition & Limestone Branch & 50.5 \\
\hline Cleveland Whiskey the Elonty Seven & Cleveland Whiskey & 43.5 & Yellowstione Select & Limestone Branch & 46.5 \\
\hline Collabor\&tion, Brandy Finish & Bardstown Bouroon $\mathrm{Co}$. & 56.5 & & & \\
\hline Collabor\&tion, Mstelle Finish & Bardstown Bouroon $\mathrm{Co}$. & 47.0 & AMERICAN WHISKEY: Malt, Rye, Tennes & e, and Wheat Whiskeys & \\
\hline Cooper's Craft" & Brown-Forman & 50.0 & Bemheim Original Wheat Whiskey & Old Pogue & 45.0 \\
\hline George T Stagg & Euffalo Trace & 64.6 & Rabolt Hole, Rye" [7a] & Rabbit Hole & 56.1 \\
\hline Heaven HIll, 6-Year Bottled-In-Bond [1n, s9i] & Heaven HI & 50.0 & Sazerac Rye 18 Year Old & Burfalo Trace & 45.0 \\
\hline LW. Harper & Dlageo & 41.0 & Thomas H. Handy & Buftaio Trace & 63.6 \\
\hline LW. Harper, 15 year [ 11$]$ & Dlageo & 43.0 & Woodford Reserve Malt Whiskey & Brown-Forman & 45.2 \\
\hline Jefferson's Chef's Collaboration & Kentucky Artsan & 45.0 & & & \\
\hline Jefferson's Ocean Aged & Kentucky Artsan & 45.1 & OTHER SAMPLES (13) & & \\
\hline Jim Beam Single Barrel [11, s9r] & $\mathrm{Jm}$ Beam & 47.5 & Arroeg, 10 year (Scotch whisky) & Glenmorangle $\mathrm{Co}$. & 46.0 \\
\hline Knob Creek & $\mathrm{Jm}$ Beam & 50.0 & Canadian Mst (Canadan welaky) [S7b, Ssa ] & Brown-Forman & 40.0 \\
\hline Maker's Mark Cask Strength [10] & Maker's Mark & 55.2 & Crown Roya (Caradlyn whiky) & Dlageo & 40.0 \\
\hline O.K.L., 10 year $[7 d]$ & New Rill & 48.9 & Deanston (Scobch whisky) & Distel Group & 46.3 \\
\hline Q.K.L. 12 year & New Rifl & 48.9 & Early Times (Kentucky whiskey) & Brown-Forman & 40.0 \\
\hline O.K.L., 8 year [10] & New Rill & 55.2 & Glen Garloch, 12 year (Scotch whisky) & Glen Garloch & 48.0 \\
\hline Oid Forester 1920 Prohliblition Style & Brown-Forman & 57.5 & Hennessy (cognas) & Jas Hennessy $8 \mathrm{Co}$. & 40.0 \\
\hline Rabbit Hole, Straight Bourbon" & Rabbilt Hole & 55.1 & Bardstown Bourbon Company" & Bardstown Bourbon Co. & 60.0 \\
\hline Russel's Reserve Single Barrel & Wid Turkey & 55.0 & Brown-Forman" [s5] & Brown-Forman & 70.0 \\
\hline Russel's Reserve Small Batch 10 Year & Wid Turkey & 50.0 & Bufflalo Trace [37a] & Buffalo Trace & 62.5 \\
\hline Stagg Jr. & Buffalo Trace & 65.0 & Umestone Branch" & Limestone Branch & 55.0 \\
\hline Town Branch Bourbon & Altech Lexngton & 40.0 & Widemess Tral" & Wildemess Trall & 68.5 \\
\hline
\end{tabular}

Highilightod sampios did not form whiskey wobs with a $20 \%$ ABV $1.0 \mu \mathrm{L}$ drop

(a) Intormution determined from package informution where possibe and from thro-party webstes when necessary.

"Sample strapht from the barrel provited by oistlen.

No fhanclal support for this research was proviled by any of the companies numed. 


\section{$\underline{\text { APPENDIX B }}$}

$\underline{\text { List of 3D Printed Parts }}$

\begin{tabular}{|l|c|c|c|c|}
\hline Part Name & $\begin{array}{c}\text { Infill } \\
(\boldsymbol{\%})\end{array}$ & Quantity & $\begin{array}{c}\text { Print Time } \\
\text { (hrs.) }\end{array}$ & $\begin{array}{c}\text { Total } \\
\text { (hrs.) }\end{array}$ \\
\hline L Bracket & 40 & 19 & 1.5 & 28.5 \\
\hline Camera Mount Adapter & 20 & 1 & 4.0 & 4 \\
\hline Lead Screw Base & 20 & 1 & 0.9 & 0.9 \\
\hline Light Mount & 20 & 1 & 3.6 & 3.6 \\
\hline Motor Calibration Datum & 20 & 1 & 1.3 & 1.3 \\
\hline Motor Mount & 20 & 1 & 2.8 & 2.8 \\
\hline Motor Shaft Adapter & 20 & 1 & 1.1 & 1.1 \\
\hline Pinch Roller Holder & 20 & 1 & 2.3 & 2.3 \\
\hline Pinch Roller Pivot & 20 & 1 & 1.5 & 1.5 \\
\hline Printer Holder & 20 & 1 & 4.5 & 4.5 \\
\hline Reel Mount & 20 & 1 & 2.8 & 2.8 \\
\hline Spring Compressor & 20 & 1 & 1.8 & 1.8 \\
\hline Supply Reel Cap & 20 & 1 & 2.9 & 2.9 \\
\hline Supply Reel Holder & 20 & 1 & 3.2 & 3.2 \\
\hline Supply Reel Brake & 20 & 2 & 0.1 & 0.2 \\
\hline Supply Reel Spacer & 100 & 1 & 1.5 & 1.5 \\
\hline Tension Test Weight & 20 & 1 & 1.0 & 1 \\
\hline Uptake Reel Cap & 100 & 1 & 1.8 & 1.75 \\
\hline Uptake Reel Gear & 20 & 1 & 1.0 & 1 \\
\hline Uptake Reel Mount & 20 & 1 & 1.6 & 1.6 \\
\hline Uptake Reel & 20 & 1 & 2.3 & 2.3 \\
\hline Total & & & $\mathbf{7 0 . 5 5}$ \\
\hline
\end{tabular}




\section{APPENDIX C}

\section{Detailed Apparatus Assembly}

An 18"x36" optical bread board (Newport SA Series) was used as the base of the test apparatus, allowing for easy alignment and modular placement of the components. Three pieces of 1.5 " square aluminum t-slotted framing rails, two feet long, were mounted vertically to the board using 3D printed L-brackets, as shown in Figure 45. These three rails will be referred to as the major mounts and their spacing on the bread board is shown in Figure 46. The major mounts can be further distinguished as the uptake mount, imaging mount, and supply mount (left, middle, and right, respectively). The three major mounts were also accompanied by two minor mounts which are shown transparently to ensure adequate space is left for the final steps of assembly. T-slotted framing rails were desirable for prototyping for their modularity. 


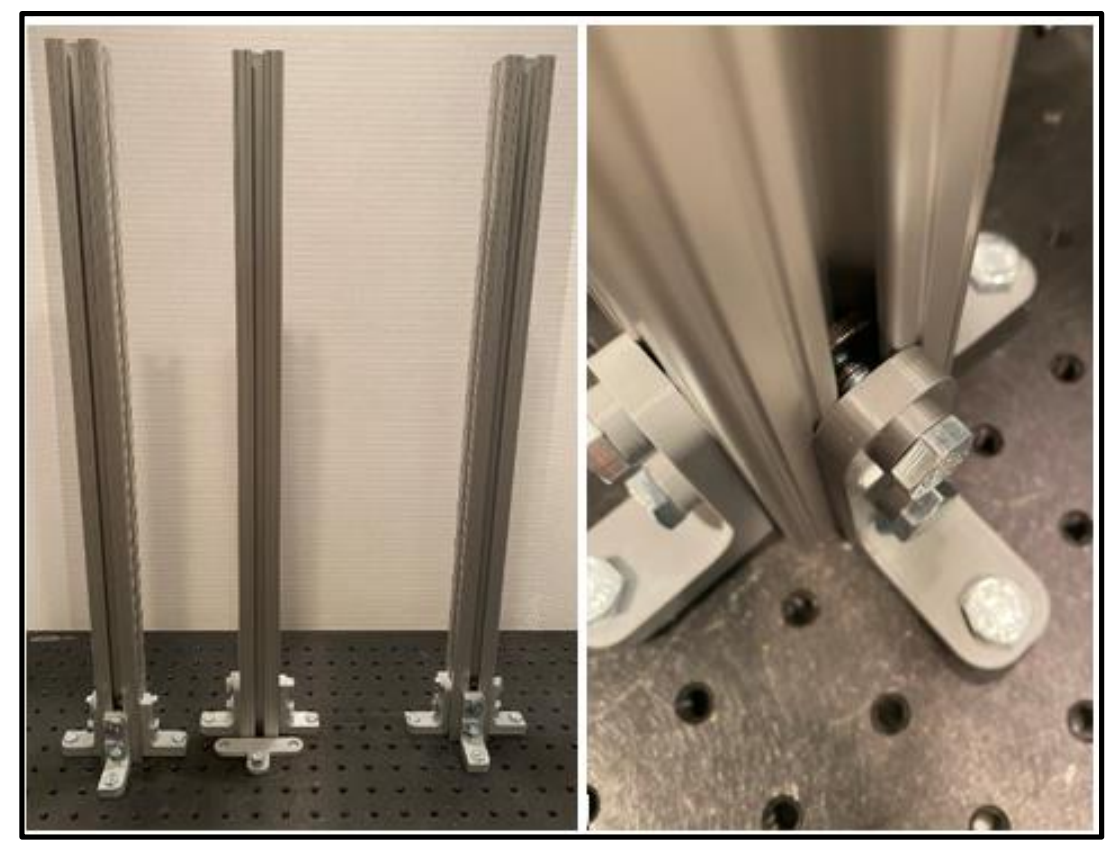

Figure 45. Assembly of major mount to optical bread board.

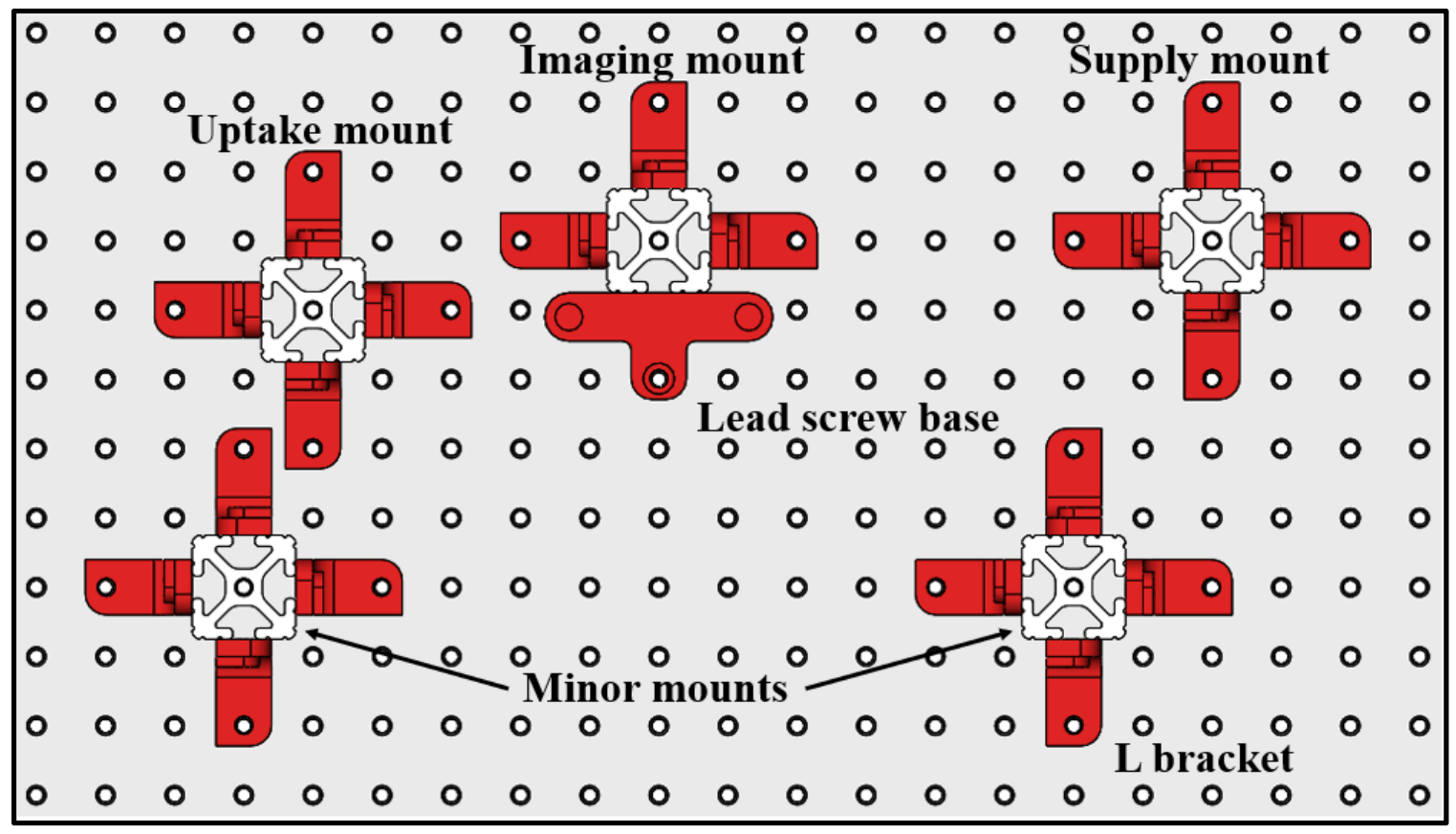

Figure 46. Spacing major and minor mounts on an optical

bread board with one-inch hole spacing. 
Note that the bottommost side of the imaging mount in Figure 46 was not one of the L-brackets supporting the other mounts. Instead, this piece was a 3D printed base for the lead screws that were used to coarsely adjust the focus of the camera once mounted. Also, illustrations do not depict the means for fastening, i.e. bolts and nuts. Figure 47 demonstrates how the imaging subsystem is assembled and how the camera was mounted to the framing rail. It was useful to avoid having the camera mount adapter touching the optical breadboard so that there are room for one's hands to use the camera controls if necessary. Once the camera was mounted, the lens was set to the correct focal length for the desired objective lens.

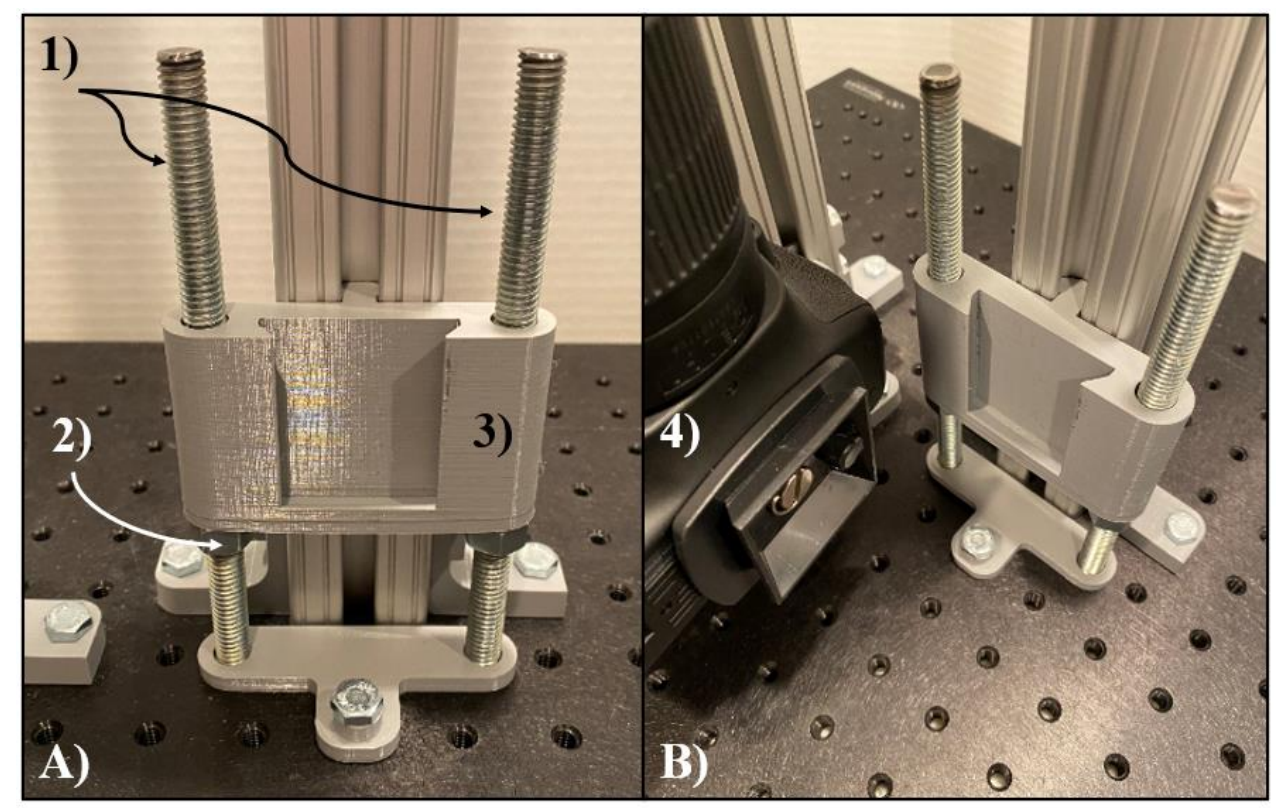

Figure 47. A) Assembly of the imaging subsystem. B) Camera being mounted to imaging subsystem. Parts used are: 1) Lead screws, 2) adjustment nut, 3) 3D printed camera mount adapter, 4) standard tripod mount attached to Canon EOS 80D. 
Next, the stepper motor was mounted to its 3D printed motor mount. In this work, a NEMA 17 hybrid stepper motor with 83.6 ounce inches of holding torque was used. The motor's specification sheet can be found in Appendix D. The motor shaft adapter and capstan were both secured to the motor's shaft using set screws, as shown in Figure 48.

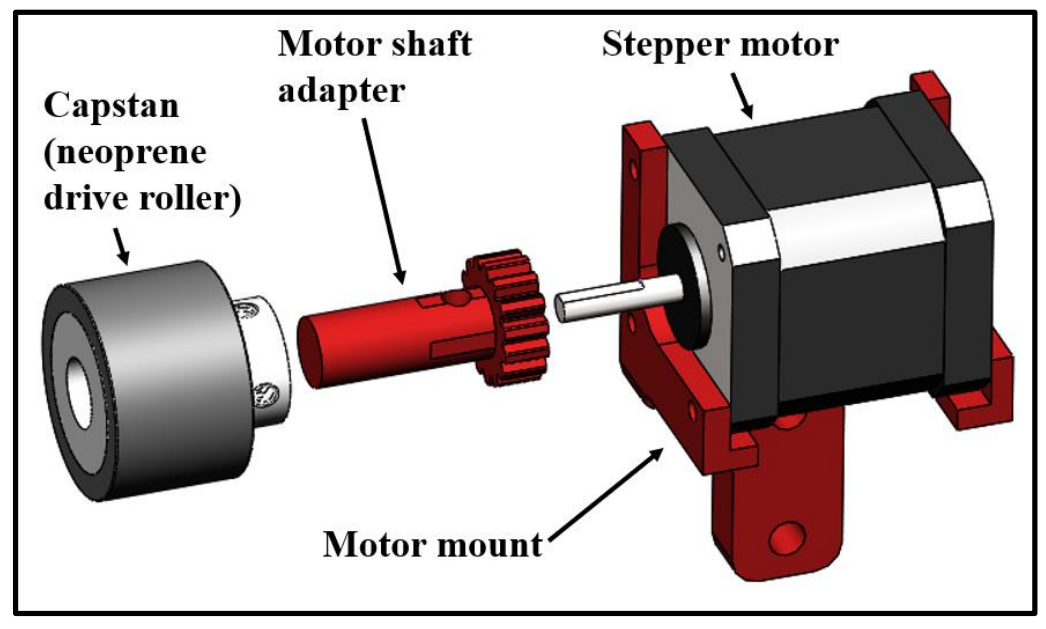

Figure 48. Exploded view of the stepper motor assembly.

The motor was secured to the right side of the uptake mount so that the top of the capstan was coincident with the focal plane of the lens. The focal plane was located at a distance from the shoulder of the objective, known as the parfocal length. Once properly the motor was properly positioned, the uptake reel subassembly was positioned to the left side of the uptake mount. The uptake reel subassembly consists of the uptake reel mount, the uptake reel gear, the uptake reel, a shaft, and a nylon sleeve bearing, and a timing belt, as shown in Figure 49A. 


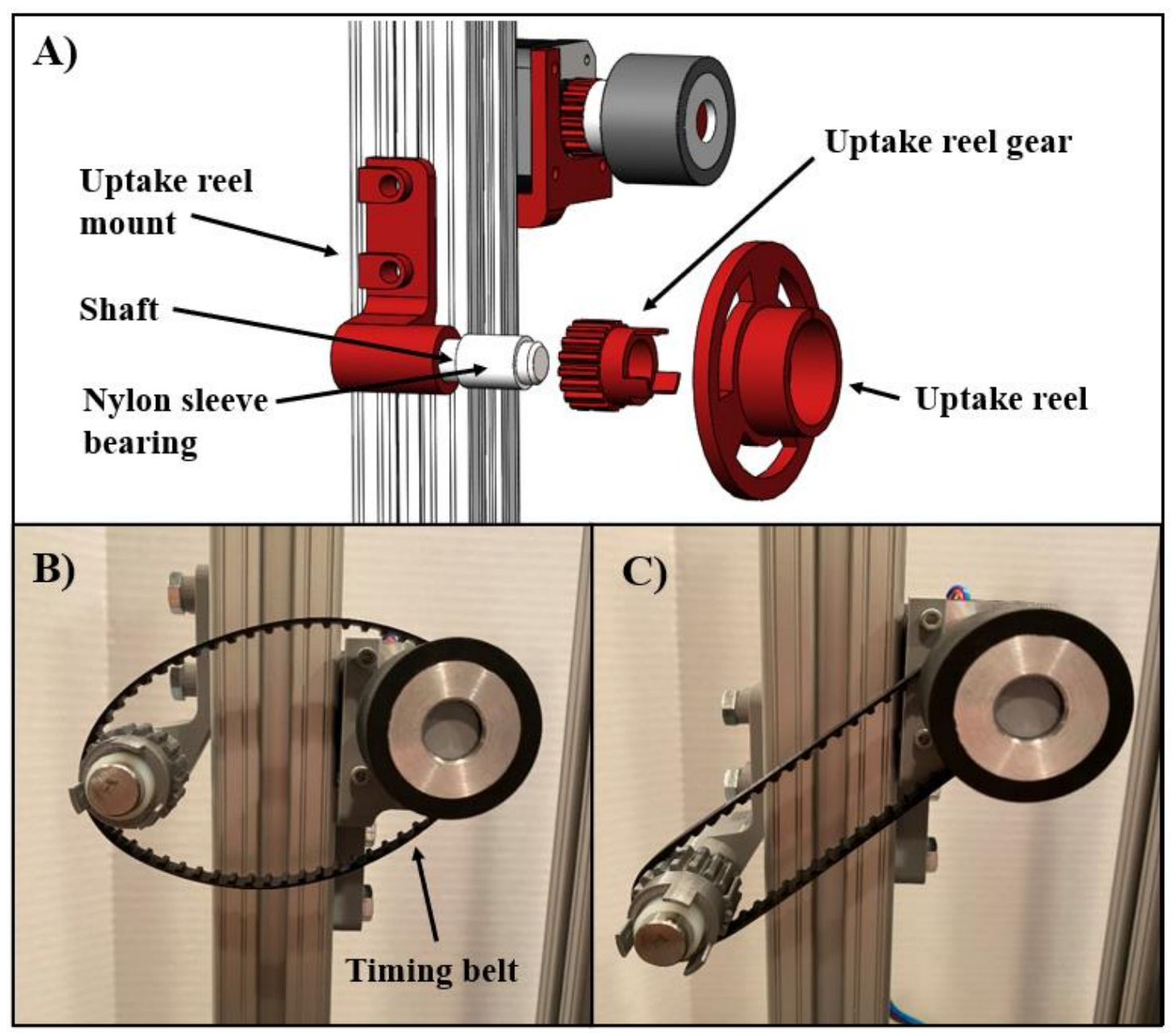

Figure 49. A) Exploded view of uptake reel subassembly. B) Timing belt being aligned with uptake reel gear and motor shaft adapter. C) Uptake reel subassembly being positioned to induce tension in the timing belt.

The uptake reel subassembly was positioned horizontally to the stepper motor so that the timing belt could be slipped over the capstan and uptake reel gear (Figure 49B). Once the teeth of the timing belt were aligned with the teeth of the two gears, the uptake reel subassembly was moved down the framing rail and secured once the timing belt was slightly pulling on the motor. The timing belt must not be pulled too taut to prevent damage to the motor from excessive lateral loading. 
Next, the supply reel holder was secured to the supply mount. As shown in Figure 50, a shaft with a nylon sleeve bearing was inserted into the supply reel holder and was leveled with the capstan. This leveling was necessary to ensure the force of gravity only acts perpendicularly to the film as the droplet travels across the tape transport system.

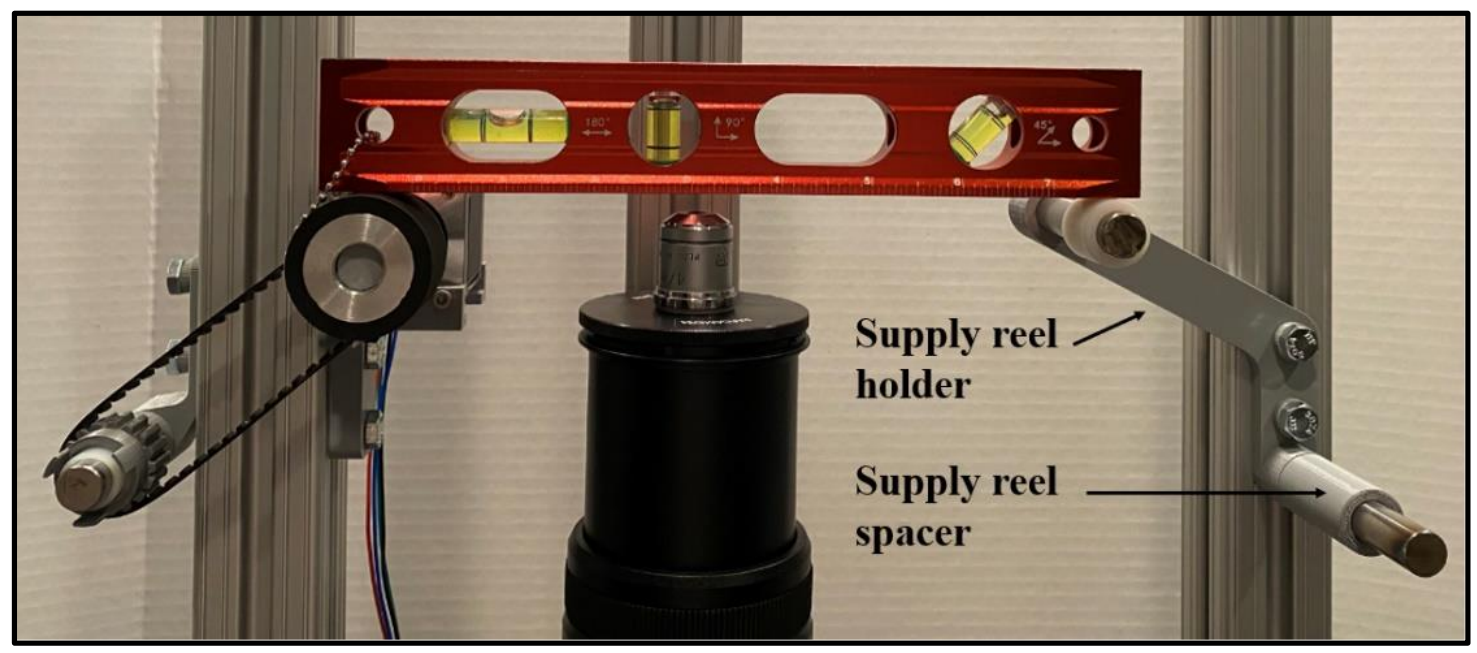

Figure 50. Leveling of supply reel holder with capstan.

The roll of FEP film was mounted to the supply reel so that the film fell to the right of the apparatus. The supply reel was then mounted to the supply reel holder, abutting the supply reel spacer, depicted in Figure 51. 


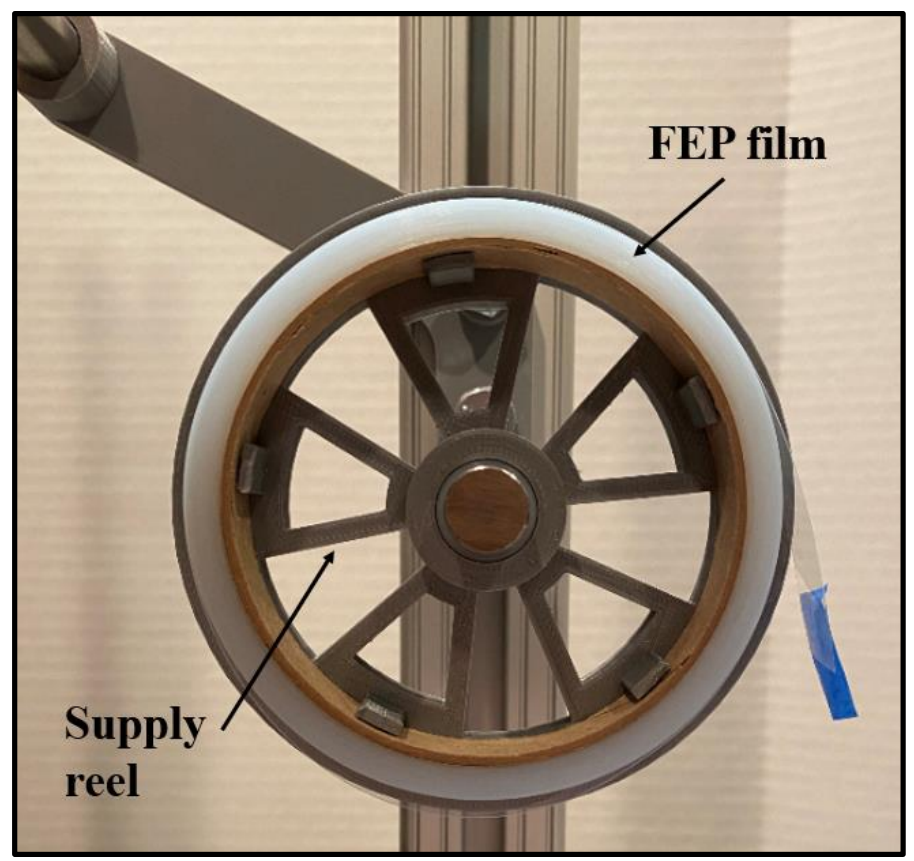

Figure 51. FEP film mounted to the supply reel holder so that the

film fell from the right side of the reel.

To prevent the supply reel from sliding off the axle, the supply reel cap was placed over the exposed end of the shafts. The supply reel cap also acted as the mount for the supply reel brakes. As illustrated in Figure 52, a t-slotted framing rail end-feed nut was positioned in a pocket and a bolt was threaded through the back side of the supply reel cap. Self-adhesive clear rubber bumpers were adhered to the tops of the supply reel brakes and they were placed atop the bolt shaft. The supply reel cap was placed onto the shafts and was held in place by the minor mount, positioned as depicted in Figure 46. 


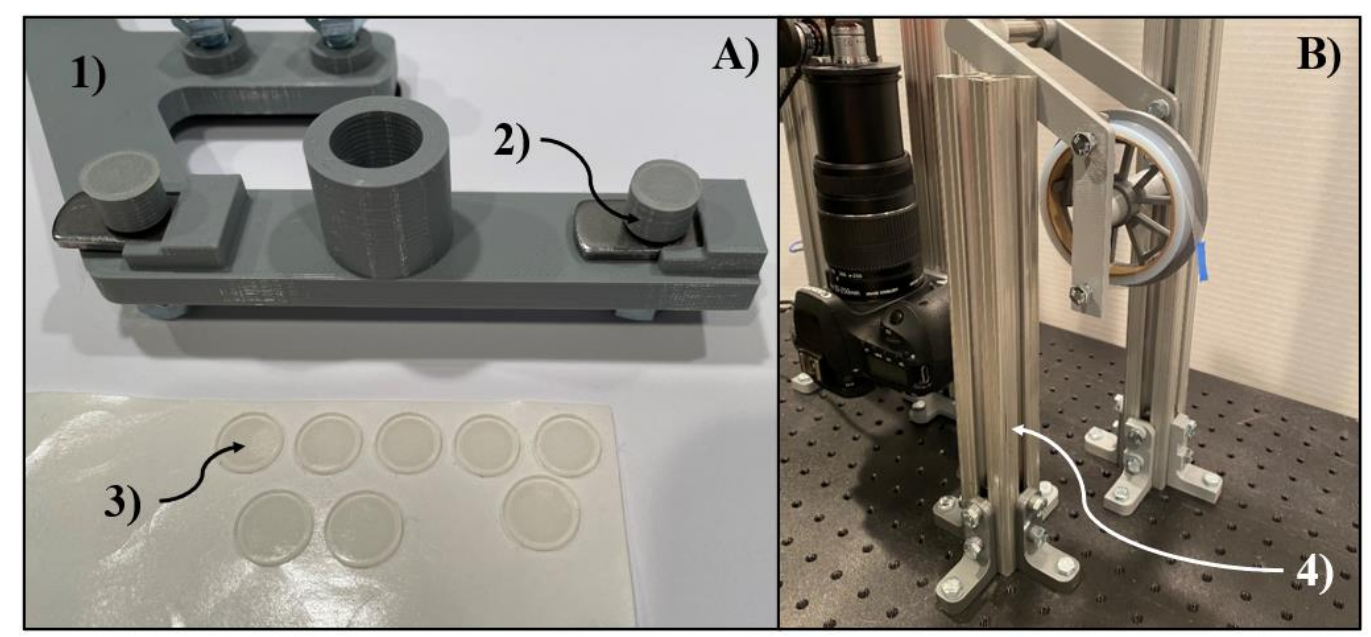

Figure 52. A) Assembly of the braking subsystem for the reel. B) Braking system being mounted to apparatus. Parts used are: 1) 3D printed supply reel cap, 2) 3D printed supply reel brake, 3) self-adhesive clear rubber bumpers, and 4) minor mount for supply reel.

The film was threaded through the system and secured to the uptake reel, as illustrated in Figure 53. From the supply reel, the film passed over the nylon sleeve bearing and then over the capstan to the uptake reel. There was a small slit in the uptake reel through which the film passed and was attached to the inner wall of the uptake reel using an adhesive tape.

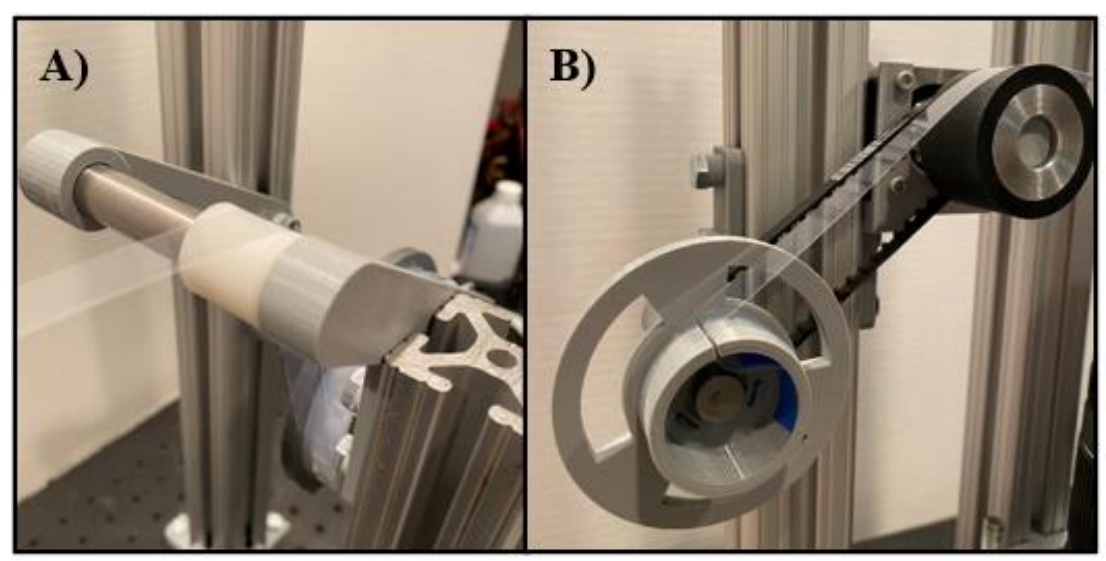

Figure 53. Illustration of tape being threaded through system. A) Film passing over nylon sleeve bearing. B) Film passing over capstan and secured to uptake reel. 
To prevent the uptake reel from sliding off the axle, the uptake reel axle cap was positioned over the uptake reel axle, as shown in Figure 54. The uptake reel axle cap was mounted to the second minor mount and once aligned with the uptake reel, secured to the optical bread board.

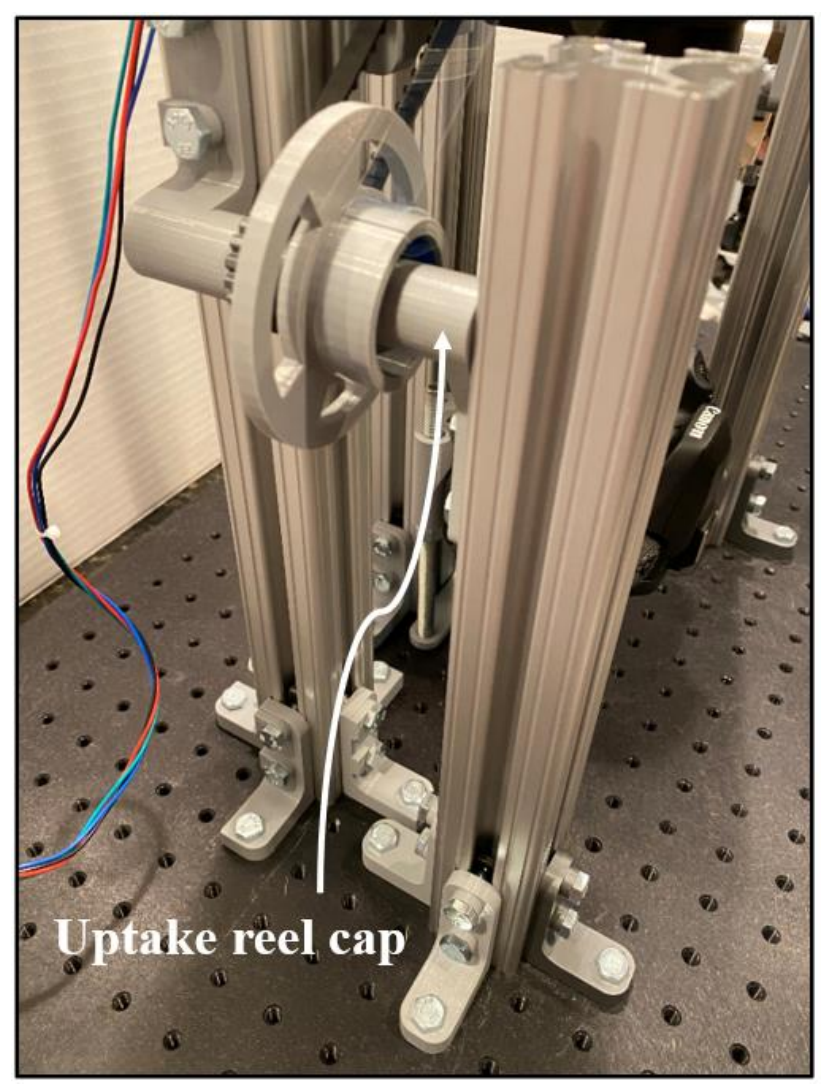

Figure 54. Uptake reel cap securing uptake reel to shaft.

Once the film was secured to the uptake reel, the pinch roller was installed to prevent the film from slipping when the motor turned. Depicted in Figure 55, the pinch roller was coupled to the pinch roller holder via a shaft. The pinch roller pivot was secured to the uptake mount so that the pinch roller holder arm was held horizontal once coupled. Compression springs were slid onto a shaft which was seated in a depression in the pinch roller holder. The spring compressor was used to compress the springs to maintain pressure 
between the roller and capstan. The spring compressor should not be overly loaded so that excessive lateral load is applied to the motor shaft.

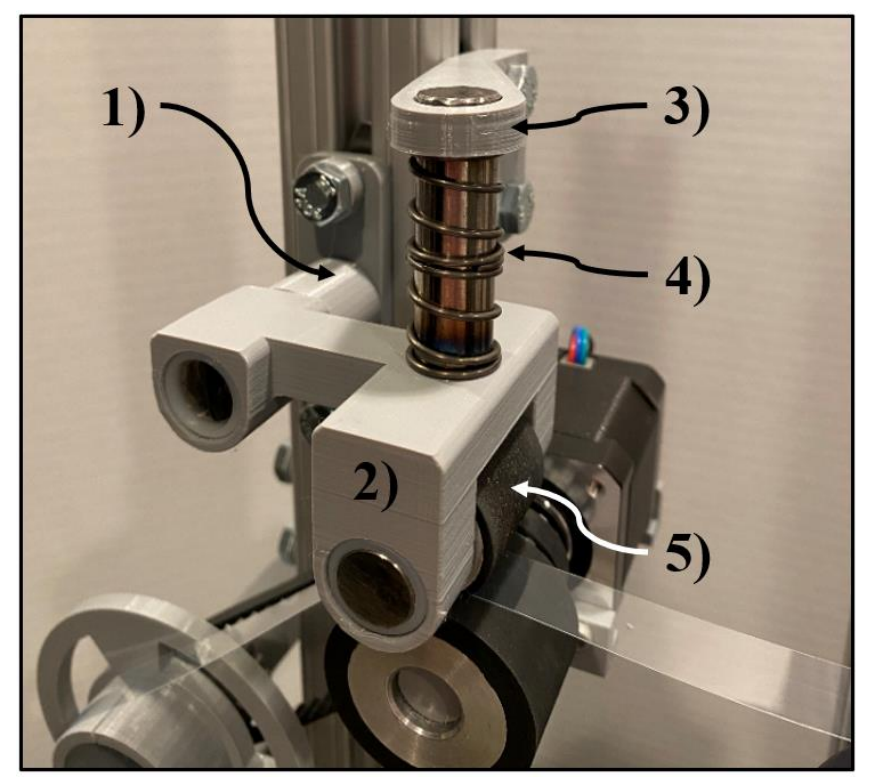

Figure 55. 1) 3D printed pinch roller pivot, 2) 3D printed pinch roller holder, 3) 3D printed spring compressor, 4) compression spring, 5) neoprene pinch roller.

Lastly, the light mount was secured above the film on the imaging mount and the printer holder was fastened to the supply mount. The printer holder was positioned so that the FCL Controller was parallel to the film, as shown in Figure 56. 


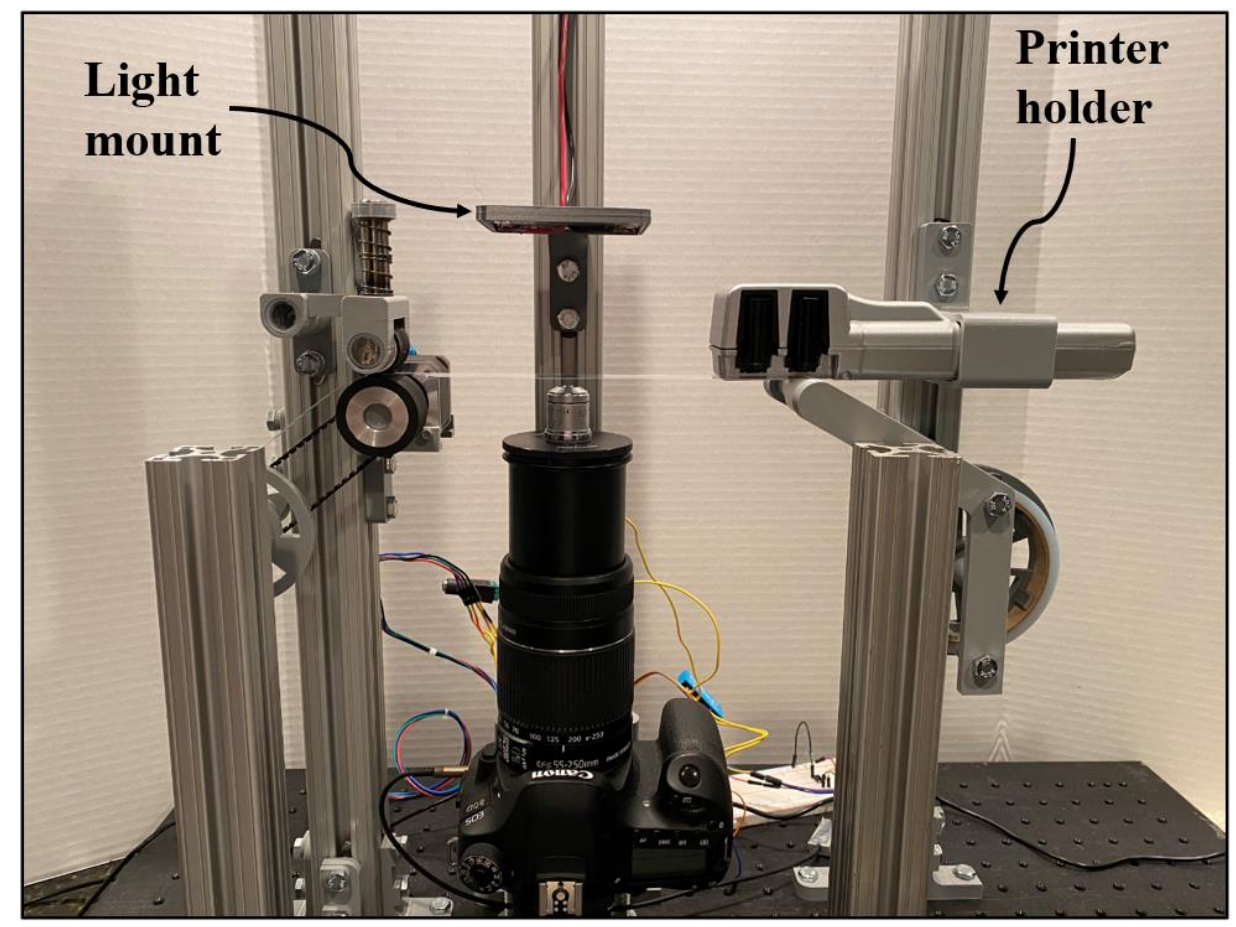

Figure 56. Final assembly of reel-to-reel apparatus. 


\section{APPENDIX D}

Stepper Motor Specification Sheet

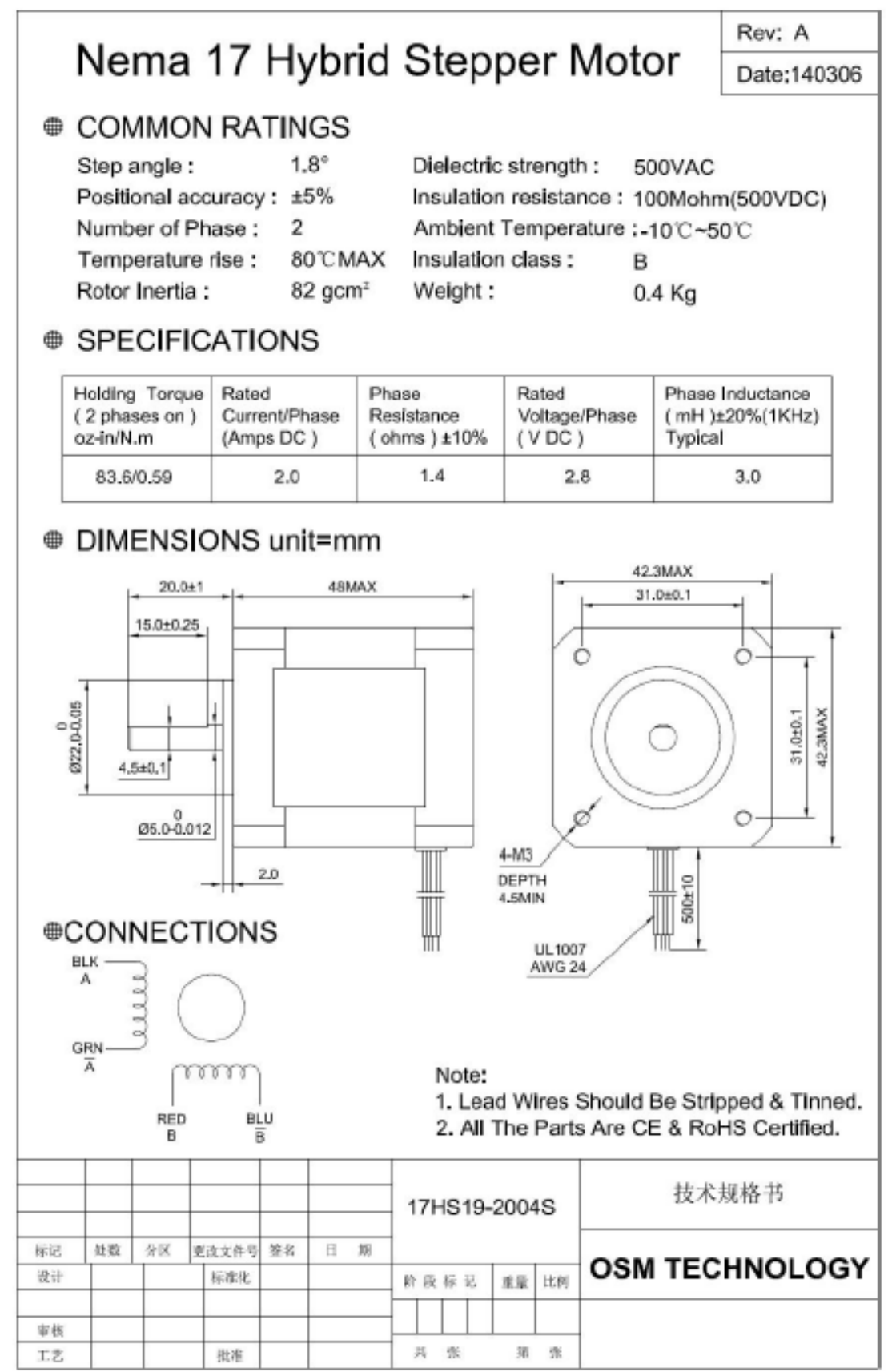


4 Wires Bi-Polar Full Step

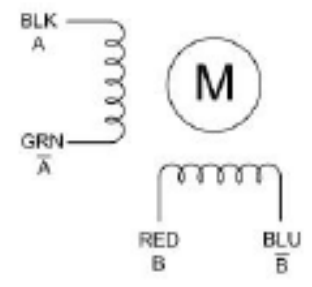

\begin{tabular}{|c|c|c|c|c|c|}
\hline $\begin{array}{c}\text { CW } \\
\text { DIRECTION }\end{array}$ & STEP & $\mathrm{A}$ & $\overline{\mathrm{A}}$ & $\mathrm{B}$ & $\overline{\mathrm{B}}$ \\
\hline \multirow{4}{*}{} & 1 & + & & + & \\
\hline & 2 & & + & + & \\
\cline { 2 - 6 } & 3 & & + & & + \\
\cline { 2 - 6 } & 4 & + & & & + \\
\cline { 2 - 6 } & 1 & + & & + & \\
\hline
\end{tabular}

- oirection of rotation as viewed from mounting end of motor

17HS19-2004S PULL OUT TORQUE 2.0A/PHASE 24V CONSTANT CURRENT HALF STEP

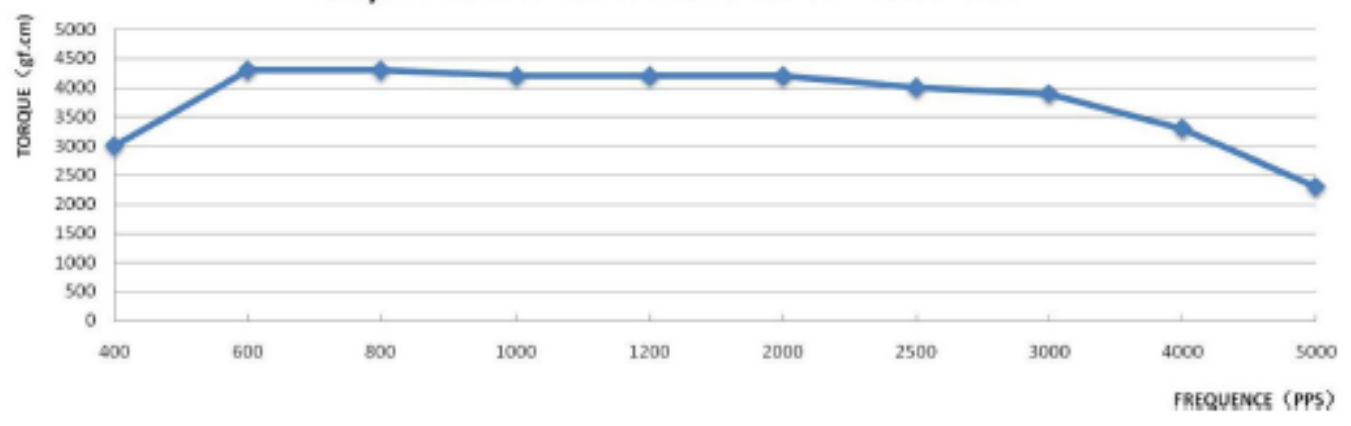




\section{APPENDIX E}

\section{Master Code}

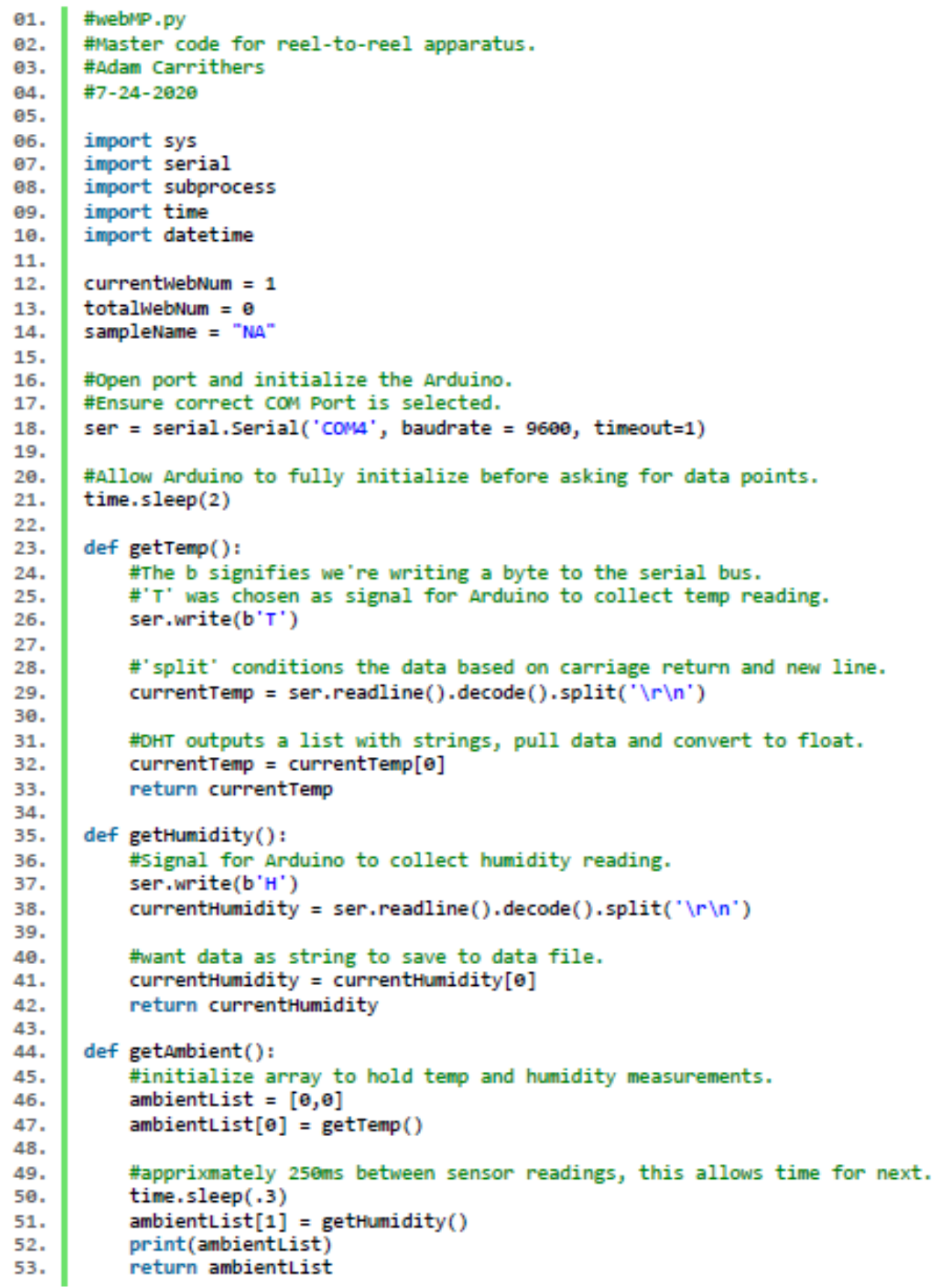




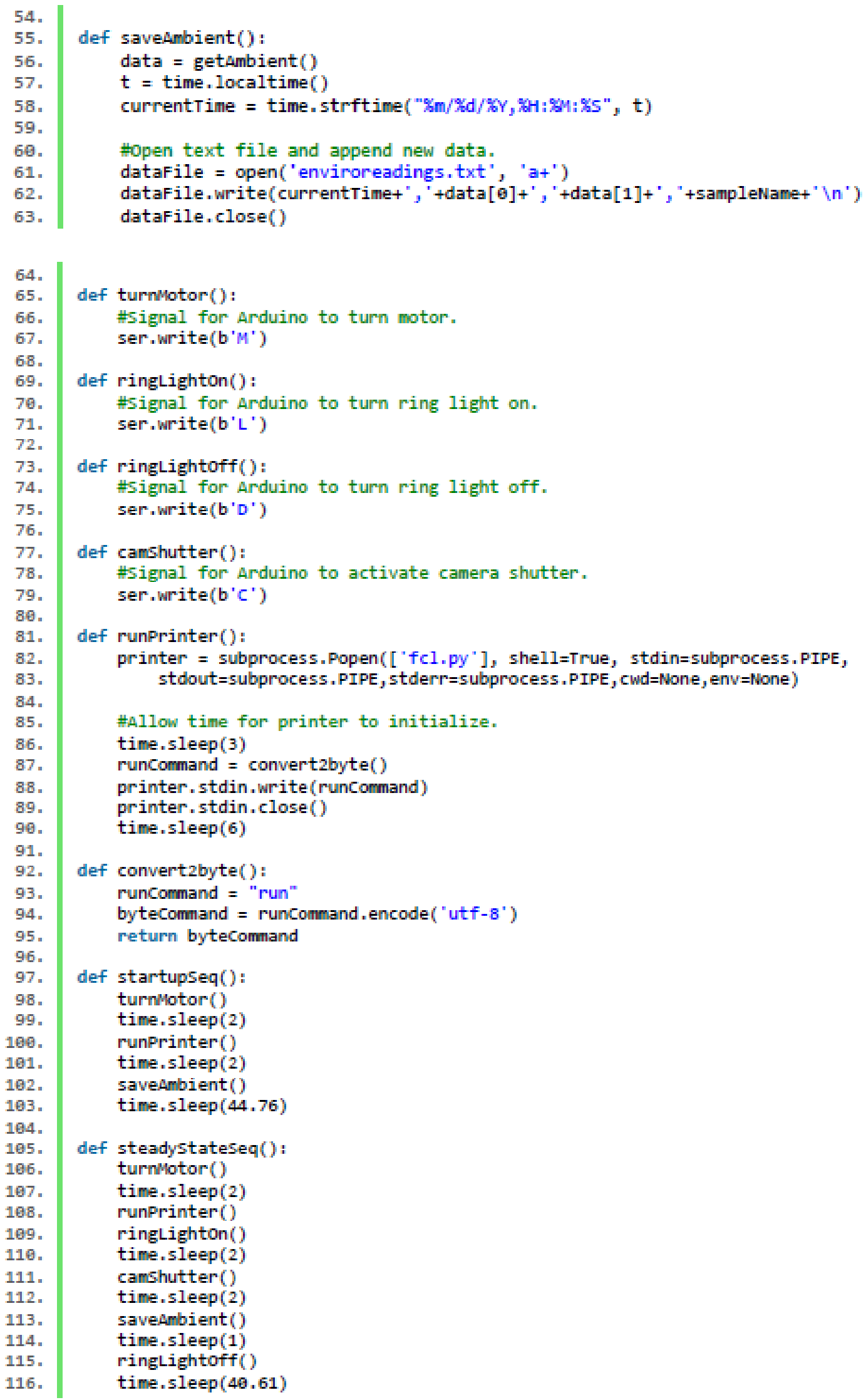




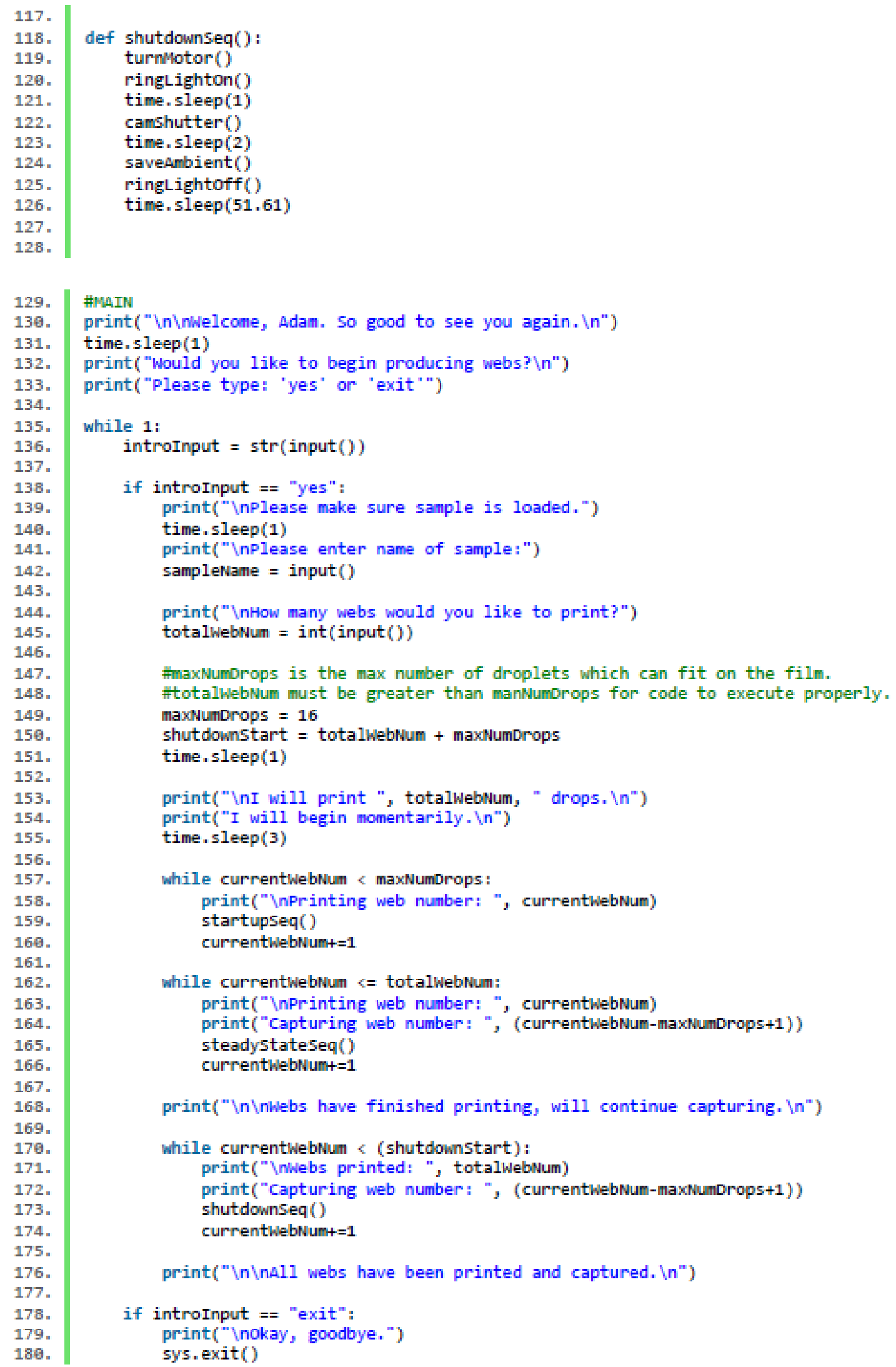




\section{APPENDIX F}

\section{$\underline{\text { Arduino Code }}$}

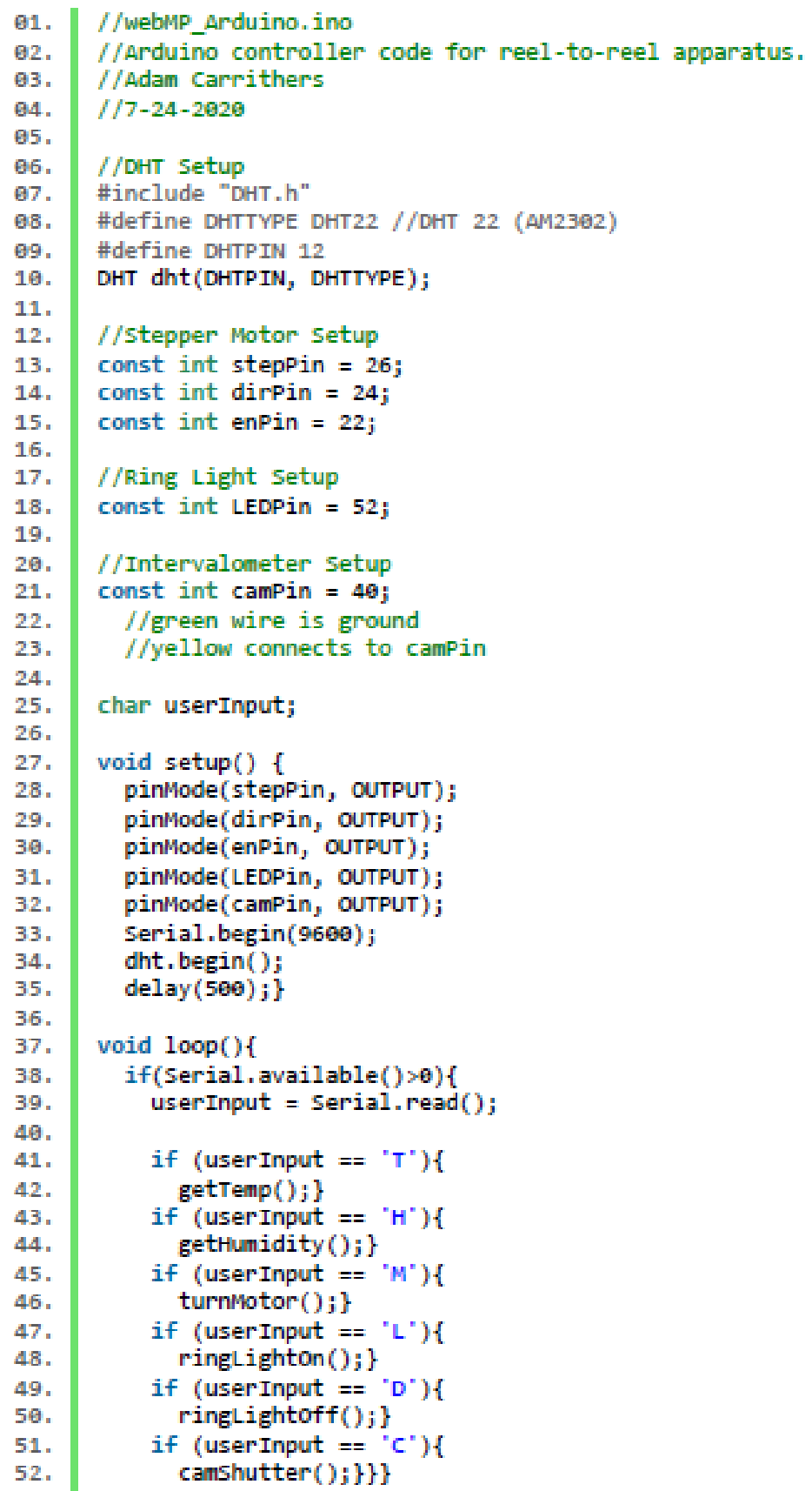




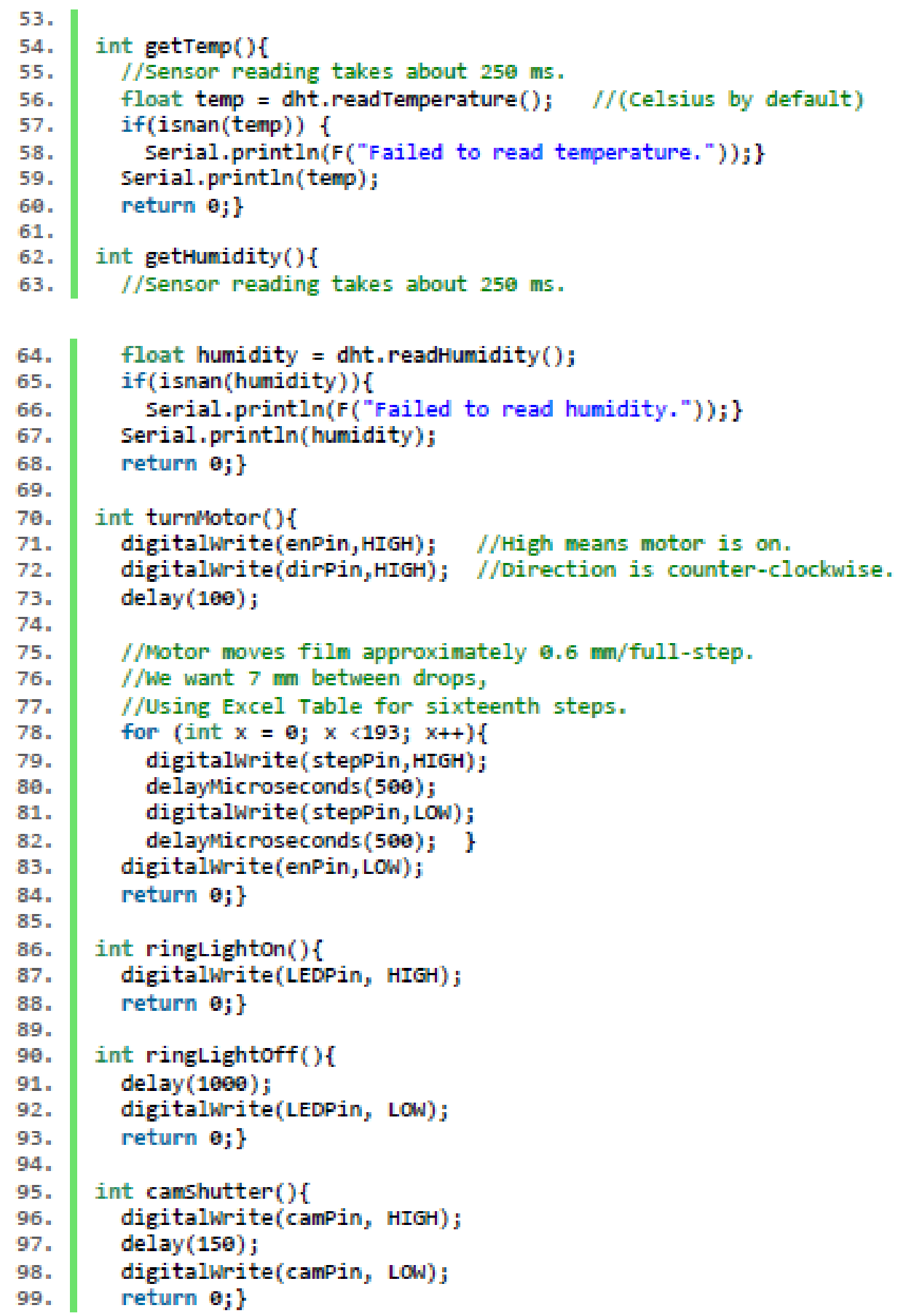




\section{CURRICULUM VITAE}

NAME:

ADDRESS:

DOB:

\& TRAINING:
EDUCATION

Adam David Carrithers

Department of Mechanical Engineering

332 Eastern Pkwy

J. B. Speed School of Engineering \& Honors College

University of Louisville

Louisville, KY 40208

Louisville, KY - January 21, 1997

B.S., Mechanical Engineering

University of Louisville

2015-2019

M.Eng., Mechanical Engineering

University of Louisville

2019-2020

AWARDS:

APS Division of Fluid Dynamics Milton van Dyke Award - 2018 Mechanical Engineering Departmental Alumni Award - 2020

Executive Vice President for Research and Innovation Grant, UofL

PROFESSIONAL The American Society of Mechanical Engineers (ASME)

SOCIETIES:

Institute of Food Technologists (IFT)

American Physics Society (APS)

PUBLICATIONS: Whiskey webs: Microscale "fingerprints" of bourbon whiskey

Physical Review Fluids - 2019

Multiscale Self-Assembly of Distinctive Web-like Structures from Evaporated Drops of Dilute American Whiskeys

ACS Nano - 2020

PRESENTATIONS: Whiskey Webs: Analysis of Self-Assembled Microstructures from Evaporated Drops of Bourbon Whiskey

APS Division of Fluid Dynamics $71^{\text {st }}$ Annual Meeting - Nov. 2018

ACCelerate Festival - April 2019

Kentucky Science Center - Eat, Drink, Do Science - Sept. 2018 